\title{
Versuche zur teilweisen Vorspannung für Längsschub und Querbiegung in Druckplatten von Betonträgern
}

\section{Working Paper}

Author(s):

Bacchetta, Aldo; Bachmann, Hugo

Publication date:

1977

Permanent link:

https://doi.org/10.3929/ethz-a-000125232

Rights / license:

In Copyright - Non-Commercial Use Permitted

Originally published in:

Bericht / Institut für Baustatik und Konstruktion ETH Zürich 6504(9) 
Versuche zur teilweisen Vorspannung für Längsschub und Querbiegung in Druckplatten von Betonträgern

Aldo Bacchetta

Hugo Bachmann 


\section{Versuche zur teilweisen Vorspannung für Längsschub und Querbiegung in Druckplatten von Betonträgern}

von

Dipl. Ing. A. Bacchetta

Prof. Dr. H. Bachmann

Institut für Baustatik und Konstruktion

Eidgenössische Technische Hochschule Zürich 
1.1 Allgemeines

1.2 Zielsetzung

1.3 Bemessungsmodelle

1.3.1 Längsschub allein

1.3.2 Längsschub mit Querbiegung und Quervorspannung

1.4 Versuchsplanung

1.4.1 Rückblick auf die Versuchsträger Q1 bis Q5 4

1.4 .2 Versuchsträger $Q 6$ und $Q 7$

\section{VERSUCHSTRAEGER}

2.1 Beschreibung

2.1.1 Abmessungen, Armierungen

2.1.2 Herstellung, Vorspannung, Lagerung 6

2.2 Baustoffe

2.2.1 Armierungsstah1, Spannstahl 7

2.2 .2 Beton

2.2.3 Zementmörtel

2.3 Bemessung und rechnerische Werte

2.3.1 Berechnete Lasten und Schubspannungen 7

2.3.2. Stegarmierung 8

2.3.3 Plattenquerarmierung 8

2.3.4 Konstruktive Ergänzung der Modellarmierungen 9

3. VERSUCHSDURCHFUEHRUNG

3.1 Versuchsanlage 10

3.2 Versuchsablauf 10

3.3 Messungen 11

3.3.1 Lasten 11

3.3.2 Durchbiegungen 11

3.3.3 Dehnungen, Stauchungen 11

3.3.4 Risse 12

4. VERSUCHSRESULTATE 13

4.1 Allgemeines Tragverhalten 13

4.2 Biegeverhalten der Träger $\quad 14$

4.2.1 Durchbiegungen 14

4.2.2 Dehnungen der Längsarmierung 14

4.2.3 Stauchungen der Druckplatte 14

4.3 Dehnungen der Plattenquerarmierung 15

4.4 Kräfte in der Plattenquerarmierung 16

4.5 Risseverhalten 16 
5. VERGLEICHE

5.1 Vergleich der Ergebnisse mit den Modellen 17

5.2 Vergleich der Ergebnisse mit und ohne Quervorspannung 18

5.2.1 Anrisslast der Druckplatte 18

5.2 .2 Risseverhalten 19

5.2.3 Dehnungen der Plattenquerarmierung 19

5.2.4 Kräfte in der Plattenquerarmierung 19

6. FOLGERUNGEN UND EMPFEHLUNGEN

ZUSAMMENFASSUNG

RESUME

SUMMARY

VERDANKUNGEN

BEZEICHNUNGEN

LITERATURVERZEICHNIS

TABELLEN 1 - 11

$\begin{array}{llll}\text { BILDER } & 1-41 & 37\end{array}$ 


\subsection{Allgemeines}

Der Anschluss von Stahlbeton-Druckplatten an Längsstege ist vor allem im Brückenbau ein wesentliches Problem. An einem Element des z.B. als einfacher Träger gelagerten Plattenbalkens gemäss Bild 1 können in einem Schnitt I-I je nach den äuaseren Belastungen folgende Schnittgrössen auftreten:

\section{a) Längsschub allein}

Wirkt eine Belastung $P$ in der Symmetrie-Ebene des T-Trägers, so entsteht eine Längsschubkraft $\tau_{y x} \cdot d$.

\section{b) Längsschub und Querbiegung}

Wirkt ferner eine Belastung $\bar{P}$ ausserhalb des Steges, so entsteht zum Längsschub hinzu ein Querbiegemoment $M_{q}$. (Die ebenfalls auftretende vertikale Schubkraft $\tau_{y z} \cdot d$ wird in der vorliegenden Untersuchung vernachlässigt.)

\section{c) Längsschub mit Querbiegung und Quervorspannung}

Ist die Druckplatte in Querrichtung noch vorgespannt, so wirkt ausser Längsschub und Querbiegung zusätzlich noch die Vorspannkraft $V$.

Zu den Problemkreisen "Längsschub allein" und "Längsschub und Querbiegung" wurden 1974 am Institut für Baustatik und Konstruktion der ETH Zürich Versuche an insgesamt fünf Stahlbetonträgern (Q1 - Q5) durchgeführt. Die erhaltenen Resultate sind im Versuchsbericht [1] dargestellt.

Im Anschluss an die erwähnten Versuche wurde 1975 an zwei weiteren Versuchsträgern (Q6 und Q7) der Fall "Längsschub mit Querbiegung und Quervorspannung" näher untersucht (vgl. Bild 12). Der vorliegende Bericht enthält die Ergebnisse dieser beiden letzten Versuche sowie Vergleiche zu den Resultaten der früheren Versuche ohne Quervorspannung.

\subsection{Zislsetzung}

Die hier beschriebenen Versuche dienten der Abklärung des Einflusses einer teilweisen Quervorspannung von Druckplatten von Betonträgern, die durch Längsschub und Querbiegung beansprucht sind. Verschiedene Bemessungsmodelle sollten überprüft und anhand von Versuchsresultaten verglichen werden. 


\subsubsection{Längsschub allein}

Da sich das Fachwerkmodell für die Schubbemessung von Stegen recht gut bewährt hat, liegt es nahe, auch für die Schubbemessung von Druckplatten ein ähnliches Modell zu entwickeln: das Flanschfachwerkmodell. Aus den Versuchen an den Trägern Q1 bis Q5 [1] ging deutlich hervor, dass die Bemessung des Schubanschlusses Platte/Steg mit diesem Modell gegenüber der Bemessung nach dem klassischen Modell (Hauptspannungen) wesentlich zweckmässiger ist.

Die Grundidee des Flanschfachwerkmodells vermittelt Bild 2. Die horizontalen Komponenten der Druckdiagonalenkräfte des Steges müssen sich in der Druckplatte ausbreiten. Dadurch entstehen Umlenkkräfte $Z_{1}=\frac{Q}{2} \cdot \operatorname{ctg} \alpha \cdot \operatorname{tg} \beta$, welche die Platte bzw. den Anschlussbereich Platte/Steg auf Zug beanspruchen. Unter Berücksichtigung der Verhältnisse bei der Kraftausbreitung in Scheiben kann ein Ausbreitwinkel $\beta$ von ca. arctg 0.5 angenommen werden. Somit beträgt die Querzugkraft $Z$ nach Flanschfachwerkmodell pro Längeneinheit des Trägers

$$
z=\frac{Q}{4 \cdot y}
$$

Die auf diese Querzugkraft bemessene Armierung kann nun, wie dies bei den vorliegenden Versuchsträgern der Fall war, in der Druckplatte von der ersten bis zur letzten Umlenkkraft $z_{1}$, d.h. von $x=y \cdot \operatorname{ctg} \alpha+\frac{b}{2}$ bis $z u x=a+\frac{b}{2}$, also über eine Länge von $a-y \cdot \operatorname{ctg} \alpha$, eingelegt werden (vgl. Bild 2 unten).

Bei diesem Vorgehen bleiben indessen zwei Aspekte unberücksichtigt, deren Einflüsse auf die gesamte Armierungsmenge sich jedoch weitgehend kompensieren:

1. Die totale im Anschlussbereich auftretende Zugkraft ist für tg $\beta=0.5=\frac{Q}{4} \cdot \operatorname{ctg} \alpha \mathrm{mal}$ Anzahl der Zugkräfte $z_{1}$, somit $z_{\text {tot }}=\frac{Q}{4} \cdot \operatorname{ctg} \alpha \cdot \frac{a}{y \cdot \operatorname{ctg} \alpha}=\frac{Q \cdot a}{4 \cdot y}$. Wird die Armierung gemäss G1. (1) nur über eine Länge von a-y-ctg $\alpha$ eingelegt, so entspricht dies einer totalen Zugkraft von nur $\frac{Q}{4 \cdot y}(a-y \cdot \operatorname{ctg} \alpha)$. Der dadurch beim Gesamtvolumen der eingelegten Armierung begangene "Fehler" (zu wenig Gesamtarmierung) von $\frac{y \cdot \operatorname{ctg} \alpha}{a}$ ist in Bild 3 in Funktion des Verhältnisses $\frac{a}{y \cdot \operatorname{ctg} \alpha}$ für $\alpha=45^{\circ}$ aufgezeichnet.

2. Das Modell kann verfeinert werden, indem der Einfluss der Stegbreite bo berücksichtigt wird. Ein Teil der horizontalen Komponente der Stegdruckdiagonalen, entsprechend der Plattenqueschnittsfläche über dem Steg (genau genommen bis zur neutralen Achse), wird sich nicht ausbreiten und verursacht somit keine Querzugkräfte. Dadurch reduziert sich die im Anschlussbereich pro Längeneinheit auftretende Zugkraft auf

$$
z=\frac{Q}{4 \cdot y} \cdot \frac{b-b_{D}}{b} \text {. }
$$

Das ursprüngliche Modell (1) weist somit gegenüber dem verfeinerten Modell (2) zuviel Armierung auf. In Bild 4 ist der grössere Armierungsaufwand (zuviel Armierung) in Abhängigkeit vom Verhältnis $b_{0} / b$ aufgetragen. 


\subsubsection{Längsschub mit Querbiegung und Quervorspannung}

Zur Erfassung des Lastfalls "Längsschub mit Querbiegung und Quervorspannung" kann ähnlich wie beim Lastfall "Längsschub und Querbiegung" vorgegangen werden (vgl. [1]).

Selbstverständlich hat die Vorspannung einen wesentlichen Einfluss auf das Verhalten im Gebrauchszustand. Eine wichtige charakteristische Grösse ist der Vorspanngrad ([3]). Auf diese Aspekte wird im folgenden Abschnitt 1.4 eingetreten. Hier soll nur die plastische Bemessung dargestellt werden.

Bei einer plastischen Bemessung kann die vorgespannte Armierung wie eine schlaffe Armierung behandelt werden, natürlich unter Berücksichtigung der viel höheren Fliessbzw. Streckgrenze. Die Plattenquerarmierung kann nach einem der folgenden zwei Modelle bestimmt werden (Bild 5):

\section{a) Superposition der Armierungen aus Flanschfachwerkmodell und aus Querbiegung}

Der Fliesswiderstand der in der Platte je zur Hälfte oben und unten einzulegenden Armierung für Längsschub wird für die Zugkraft $Z$ gemäss Flanschfachwerkmodell berechnet. Derjenige für das Querbiegemoment $M_{q}$ wird nach der normalen plastischen Biegetheorie bestimmt. Beide Fliesswiderstände bzw. Armierungen werden nun überlagert. Benötigt wird also eine obere und eine untere Armierungslage, wobei die obere aus einer vorgespanten und einer schlaffen Armierung besteht (Bild 5a).

\section{b) Armierung für Querbiegung mit Querzugkraft aus Flanschfachwerkmodell}

Die axiale Querzugkraft (aus Flanschfachwerkmodell) und das Querbiegemoment $M_{q}$ werden als gemeinsam wirkende Querschnittsbeanspruchung betrachtet. Der Fliesswiderstand der erforderlichen Armierung (vorgespant und schlaff) wird darauf nach den Regeln der plastischen Theorie ermittelt. Demgemäss wird im Querschnitt eine obere Armierung benötigt, während unten (sofern das Querbiegemoment gross genug ist) eine Druckkraft entsteht und somit keine Armierung notwendig wird (Bild 5b).

Ein Vergleich der beiden Bemessungsmethoden a) und b) zeigt folgendes:

1. Das Modell a) ergibt oben die gleiche Zugkraft $K_{0}$ bzw. Armierung $F_{e}+F_{s}$ wie das Modell b), sofern der gleiche Hebelarm der inneren Kräfte $y_{p}$ angenommen wird.

2. Das Modell a) führt unter dieser Voraussetzung (gleiches $y_{p}$ ) auch unten zum gleichen Resultat wie das Modell b), sofern die untere Zugkraft aus dem Flanschfachwerkmodell mit der Druckkraft aus Querbiegung "verrechnet" wird.

Insbesondere bei dünnen Platten wird es oft genügen, die Bemessung gemäss dem einfacheren Modell a) mit Verrechnung der Kräfte in der Querbiege-Druckzone vorzunehmen. 
1.4.1 Rückblick auf die Versuchsträger Q1 bis Q5 [1]

Träger Q1:

Der Versuchsträger Q1 wurde nur einer Längsbelastung $P$ unterworfen. Somit entstanden im Anschlussbereich Platte/Steg Längsschubspannungen $\tau_{y x}$. Nach der klassischen Theorie wurden die Hauptzugspannungen $\sigma_{h z}$ berechnet und eine entsprechende Armierung eingelegt.

Träger Q2:

Auch dieser Träger wurde nur in Längsrichtung belastet. Die Beanspruchung bzw. Armierung im Anschlussbereich wurde jedoch nach dem Flanschfachwerkmodell ermittelt.

Träger Q3:

Beim Träger Q3 wurde ausser der Längsbelastung $P$ noch eine Querbelastung $\bar{P}$ aufgebracht; somit wirkte im Anschlussbereich zusätzlich zur Längsschubkraft $\tau_{y x}{ }^{\circ} d$ noch ein Querbiegemoment $M_{q}$. Die Armierung wurde als Superposition der Armierungen aus Hauptzugspannungsmodeli und aus Querbiegung ermittelt.

Träger Q4:

Der Träger Q4 wurde gleich wie der Träger Q3 belastet. Die Armierung wurde jedoch als Superposition der Armierungen aus Flanschfachwerkmodell und aus Querbiegung bestimmt.

Träger Q5:

Auch dieser Träger wurde wie die Träger Q3 und Q4 belastet. Die Armierung wurde jedoch für "Querbiegung mit Querzugkraft aus Flanschfachwerkmodeli" errechnet.

Mit "Längsbelastung" wird die Belastung P zur Erzeugung des Längsschubes, mit "Querbelastung" die Belastung $\bar{P}$ zur Erzeugung des Querbiegemomentes bezeichnet. Beide Belastungs- und Beanspruchungsarten waren vällig unabhängig voneinander. Die Querbelastung $\bar{P}$ wurde so aufgebracht, dass sich im Trägerquerschnitt gewissermassen ein Eigenspannungszustand bildete. Somit erzeugte die Querbelastung keine Längsbeanspruchungen (vgl. Abschnitt 3.1).

\subsubsection{Versuchsträger Q6 und Q7}

Um das Verformungs- und Tragverhalten von Druckplatten im Anschlussbereich mit dem Steg für den Lastfall "Längsschub mit Querbiegung und Quervorspannung" zu erforschen, wurden die Versuchsträger Q6 und Q7 nach den zwei folgenden Modellen konzipiert:

Träger Q6:

Die Plattenquerarmierung wurde als Superposition der Armierungen aus Flanschfachwerkmodell und aus Querbiegung bestimmt (analog Q4, siehe Bild 5a).

Träger Q7:

Die Plattenquerarmierung wurde für "Querbiegung mit Querzugkraft aus Flanschfachwerkmodell" ermittelt (analog Q5, siehe Bild 5b).

Die Belestung und Ausbildung dieser beiden Versuchsträger lehnte sich eng an die bei den Trägern Q1 bis Q5 gemachten Erfahrungen an. Dort hatte sich das Flanschfachwerkmodell als dem klassischen Modell (Hauptzugspannungen) überlegen erwiesen, so dass 
hier nur noch das Flanschfachwerkmodell berücksichtigt wurde. Ferner wurden die Abmessungen der Träger und die Stegarmierungen unverändert übernommen. Dies empfahl sich auch im Hinblick auf einen Vergleich zwischen Trägern mit und ohne Quervorspannung (Q6 mit Q4 und Q7 mit Q5). Im übrigen ist in [1] eine ausführliche Parameteranalyse enthalten, die der Wahl der Abmessungen und Stegarmierungen zugrunde gelegt worden war.

Die Quervorspannung wurde bei beiden Trägern konstant längs der ganzen Druckplatte aufgebracht. Ihre Grösse wurde so gewählt, dass in den Bereichen "reine Querbiegung" das Fliesskraftverhältnis

$$
\lambda=\frac{F_{s} \cdot \sigma_{s f}}{F_{s} \cdot \sigma_{s f}+F_{e} \cdot \sigma_{e f}}
$$

0.60 betrug. Dies entspricht einer häufig anzutreffenden teilweisen Vorspannung von Fahrbahnplatten in Brückenquerschnitten.

Der Vorspanngrad

$$
k^{\prime}=\frac{M_{z u 1, v}}{M_{g+p}}
$$

(vgl. [3]) nahm in den Bereichen "reine Querbiegung" unter Berücksichtigung einer zulässigen Betonspannung am Zugrand $\sigma_{r}$ nach Norm SIA 162 [4] von $10 \mathrm{~kg} / \mathrm{cm}^{2}$ und einer Beanspruchung im Gebrauchszustand von $M_{g+p}=M_{p l} / 1.8$ den Wert 0.80 an.

In den Bereichen "kombinierte Beanspruchung" (Querbiegung mit Querzugkraft) ergab sich der Vorspanngrad $k^{\prime}$ für das Verhältnis $\gamma=\frac{Z}{M_{q} / d}$ von $1.5 \mathrm{zu} 0.64$.

Es ist zu bemerken, dass der Vorspanngrad $k^{\prime}$ bei relativ kleinen Vorspannkräften stark von den zulässigen Betonspannungen am Zugrand $\sigma_{r}$ abhängt. Aus Tabelle 7 ist der Einfluss der zulässigen Zugspannungen wie auch des Verhältnisses $\gamma$ auf den Vorspanngrad $k^{\prime}$ ersichtlich. 
2.1 Beschreibung

\subsubsection{Abmessungen, Armierungen}

Die Abmessungen und Armierungen gehen aus den Bildern 6 und 7 hervor.

\section{Abmessungen:}

Bei den Trägern Q6 und $Q 7$ wurden die schon bei den Trägern Q1 bis Q5 [1] verwendeten Abmessungen beibehalten.

\section{Stegarmierung:}

Auch die Biege- und Schubarmierung wurde beibehalten, nämlich 6 Ø $30 \mathrm{~mm}$ für die Biegearmierung $\left(\mu_{L}=0.77 \%\right)$ und Bügel $\emptyset 12 \mathrm{~mm}$ im Abstand $t=10 \mathrm{~cm}\left(\mu_{B}=1.13 \%\right)$ für die Schubarmierung.

\section{Plattenquerarmierung:}

Die schlaffe Plattenquerarmierung bestand aus Armierungsstäben $\oslash 6 \mathrm{~mm}$ im Abstand $t=8.5 \mathrm{~cm}$ bis $27.5 \mathrm{~cm}$, die in zwei Lagen, d.h. oben und unten in der Platte, eingelegt wurden.

Als vorgespannte Querarmierung wurden Spanndrähte $\emptyset 4 \mathrm{~mm}$ im Abstand $t=25 \mathrm{~cm} v e r-$ wendet. Um einen einwandfreien Verbund sicherzustellen und nicht ein kleines Hüllrohr injizieren zu müssen, wurde folgende Lösung gewählt (Bild 8): Beim Betonieren wurden in der Platte kanalförmige, d.h. nach oben offene sowie in Längsrichtung gerillte Aussparungen angebracht. In diese konnten die Spanndrähte eingelegt werden. Nach dem Vorspannen wurden die Aussparungen mit Zementmörtel ausgefüllt.

In mehreren Vorversuchen wurde die bestmögliche Zusammensetzung bzw. Konsistenz des Zementmörtels getestet. Die Kriterien waren einerseits ein möglichst geringes Schwinden und andererseits eine ähnliche Biegezugfestigkeit $\beta_{b z}$ wie die des Betons. Das Resultat war ein erdfeuchter Zementmörtel (Tabellen 5 und 6), welcher mit einem Handfäustel in die Aussparungen eingestampft wurde.

Die Vorspannkräfte entsprechend einer Vorspannung $\sigma_{s v}(t=0)=0.7 \beta_{z}$ wurden mit einem Dynamometer gemessen. In einem Vorversuch war mittels Dehnungsmessungen längs der Vorspanndrähte der Spannungsabfall infolge Schwinden, Kriechen und Relaxation nach 28 Tagen zu etwa $10 \%$ ermittelt worden.

\subsubsection{Herstellung, Vorspannung, Lagerung}

Die Herstellung der beiden Versuchsträger Q6 und Q7 erfolgte in einer Holzschalung. Der Beton wurde mit einem Nadelvibrator verdichtet. Fünf Tage nach dem Betonieren wurde der Träger ausgeschalt. Im Alter von 14 Tagen wurden die Vorspanndrähte angebracht und vorgespannt. Anschliessend erfolgte das oben beschriebene "Injizieren". Bis zum Prüfalter von 42 Tagen wurden die Träger in der Betonierhalle gelagert, wo sie für die Prüfung vorbereitet wurden. 


\subsubsection{Armierungsstahl, Spannstahl}

Die Biegearmierung $\emptyset 30 \mathrm{~mm}$ wie auch die Stegbügel $\emptyset 12 \mathrm{~mm}$ bestanden aus naturhartem Stahl (Box-Ultra).

Die schlaffe Querarmierung der Platte bestand aus kaltverformtem Armierungstahl $\emptyset 6 \mathrm{~mm}$ (Tor 50), welcher aber durch eine spezielle Wärmebehandlung im Werk wieder eine ausgeprägte Fliessgrenze erhalten hatte.

Die vorgespannte Querarmierung der Platte bestand aus patentiert kaltgezogenen Spanndrähten $\emptyset 4 \mathrm{~mm}$ (Stahlton $A G$ ).

Die Kennwerte der verwendeten Stähle sind in der Tabelle 1 zusammengestellt. Sie wurden in einer dehnungsgesteuerten Zugmaschine ermittelt (genaues Vorgehen siehe [1]). Der Variationskoeffizient in Prozenten ist jeweils in Klammern angegeben. Die ermittelten $\sigma-\varepsilon-D i a g r a m m e$ der beiden Plattenarmierungen gehen aus Bild 9 hervor.

\subsubsection{Beton}

Die Zusammensetzung des Frischbetons war für beide Träger gleich und ist in Tabelle 2 angegeben. Als Bindemittel wurde normaler Portlandzement verwendet.

Zur Bestimmung der konventionellen Festigkaitswerte, Würfeldruckfestigkeit $\beta_{w}$, Prismendruckfestigkeit $\beta_{p}$ und Biegezugfestigkeit $B_{b z}$, wurden Prismen $(12 \times 12 \times 36 \mathrm{~cm})$ hergestellt. Für die Ermittlung der Spaltzugfestigkeit $\beta_{q z}$ dienten Zylinder $(\emptyset 15 \mathrm{~cm}$, $H=30 \mathrm{~cm})$. Die Mittelwerte dieser im Alter von 28 Tagen geprüften Proben sind in Tabelle 3 aufgeführt.

Das $\sigma-\varepsilon$-Diagramm des verwendeten Betons wurde aufgrund der in [1] dargesteliten Ergebnisse (und in Tabelle 4 teilweise wiederholt) durch eine kubische Parabel angenähert. Es ist in Bild 10 dargestellt.

\section{$\underline{2.2 .3 \text { Zementmörtel }}$}

Die Zusammensetzung des für die "Injektion" der Spanndrähte verwendeten frischen Zementmörtels ist in Tabelle 5 angegeben. Zur Ermittlung der konventionellen Mörtelfestigkeiten $\beta_{p}$ und $\beta_{b z}$ wurden kleine Prismen $(4 \times 4 \times 16 \mathrm{~cm})$ hergestellt. Die Mittelwerte dieser im Alter von 28 Tagen, d.h. gleichzeitig mit den Versuchsträgern geprüften Proben sind aus Tabelle 6 ersichtlich.

\subsection{Bemessung und rechnerische Werte}

\subsubsection{Berechnete Lasten und Schubspannungen}

Der Bemessung der Träger wurden die mit angenommenen Materialkennwerten berechneten theoretischen Last- und Schnittkraftgrössen zugrunde gelegt (vorberechnete Werte). In der ersten und dritten Spalte der Tabelle 10 sind die entsprechenden Längs- und Querbelastungen für den Biegebruch des Trägers ( $P_{u}^{T h}$ ) und für den Fliessbeginn der Plattenquerarmierung ( $P_{f}^{T h}, \bar{P}_{f}^{T h}$ ) aufgeführt. Die bei diesen Lasten im Steg bzw. im Anschluss Platte/Steg sich ergebenden nominellen Schubspannungen sind in den Spalten zwei und vier derselben Tabelle angegeben. Diese werden wie folgt definiert: 
- Nominelle Schubspannung im Steg:

$$
\tau_{s}=\frac{Q}{b_{0} \cdot h}=\frac{P_{u}^{T h}}{b_{0} \cdot h} \quad(h=55 \mathrm{~cm} \text { : statische Höhe des Trägers })
$$

- Nominelle Längsschubspannung im Anschluss: Platte/Steg:

$$
\tau_{y x}=\frac{Q}{2 \cdot d \cdot y}\left(1-\frac{b_{0}}{b}\right)=\frac{P_{f}^{T h}}{2 \cdot d \cdot y}\left(1-\frac{b_{0}}{b}\right) \quad\left(y=50 \mathrm{~cm} \text { : } \begin{array}{l}
\text { Hebelarm der inneren Kräfte } \\
\text { des Trägers })
\end{array}\right.
$$

- Nominelle vertikale Querschubspannung im Anschluss Platte/Steg:

$$
\tau_{y z}=\frac{\bar{P}_{f}^{T h}}{\bar{l} \cdot h_{p}} \quad\left(1=88 \mathrm{~cm} \text { : Abstand der Querbelastung } \bar{P} ; h_{p}=8.7 \mathrm{~cm}\right. \text { : statische }
$$

Eine Längsbelastung $P$ von 43 t erzeugt im Anschluss Platte/Steg eine nominelle Längsschubspannung $\tau_{x y}$ von $34.2 \mathrm{~kg} / \mathrm{cm}^{2}$. Die bei den vorliegenden Versuchen nicht weiter betrachteten nominellen vertikalen Querschubspannungen $\tau_{y z}$ infolge einer Querbelastung $\bar{P}$ von 2.91 t ergaben sich $z u 3.8 \mathrm{~kg} / \mathrm{cm}^{2}$.

Für den direkten Vergleich mit den Versuchsresultaten (experimentelle Werte) enthält die Tabelle 10 ferner die mit den effektiv ermittelten Materialkennwerten (dynamische Werte) berechneten Grössen (nachberechnete Werte).

\subsubsection{Stegarmierung}

\section{Bügelarmierung :}

Da der Längsschub im Anschluss Platte/Steg mit der Querkraft zunimmt, wurden die Träger auf eine möglichst grosse Querkraft bemessen. Als "Bemessungslast" der Träger wurde daher jene Last angenommen, unter der die nominelle Schubspannung des Steges

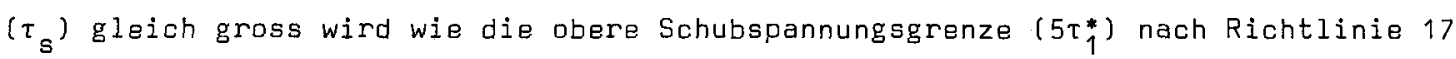
der Norm SIA 162 [4]. Bei den gewählten Querschnittsabmessungen (Bild 6) sowie einer angenommenen Würfeldruckfestigkeit des Betons von $\beta_{w 28}=300 \mathrm{~kg} / \mathrm{cm}^{2}$, wird die obere Schubspannungsgrenze bei einer Last $P_{u}^{T h}$ von 55 t erreicht (ohne Berücksichtigung des Eigengewichtes und der Belastungseinrichtungen).

\section{Längsarmierung :}

Die Biegearmierung liess sich für die gleiche Belastung $\left(P_{u}^{T h}=55 t\right)$ nach den üblichen Regeln der plastischen Bemessung bestimmen. Dabei wurde für den Stahl eine Fliessspannung von $5.2 \mathrm{t} / \mathrm{cm}^{2}$ sowie für Beton eine Würfeldruckfestigkeit von $\beta_{w 28}=$ $300 \mathrm{~kg} / \mathrm{cm}^{2}$ angenommen.

Die Stegarmierungen sind aus Bild 6 ersichtlich. Der Gehalt der Biegearmierung $\mu_{L}$ beträgt $0.77 \%$, derjenige der Schubarmierung $\mu_{B} 1.13 \%$.

\subsubsection{Plattenquerarmierung}

Die Druckplatten beider Versuchsträger können in verschieden beanspruchte Bereiche aufgeteilt werden (Bereiche I und II, Bild 11): 
- Bereiche "reine Querbiegung"
- Bereiche "kombinierte Beanspruchung" (durch Längsschub und Querbiegung).

In den Bereichen "reine Querbiegung" wurde die totale Querarmierung auf die Querbelastung $\tilde{P}_{f}^{T h}=2.91 \mathrm{t}$ pro Kolben bemessen (Bild 13). Diese im Abstand von $1=88 \mathrm{~cm}$ längs der Druckplatte wirkende Belastung ergibt im Anschluss Platte/Steg ein Querbiegemoment $M_{q}$ von $1.09 \mathrm{mt} / \mathrm{m}^{\prime}$. Die Armierung wurde nach den üblichen Regeln der plastischen Bemessung bestimmt. Der Anteil der vorgespannten Armierung kann durch das Fliesskraftverhältnis $\lambda$ beschrieben werden (siehe Abschnitt 1.4.2). Es betrug hier 0.6; entsprechend war der Vorspanngrad $k^{\prime}=0.8$ (Tabelle 7). Als Querbiegearmierung wurden Spanndrähte $\emptyset 4 \mathrm{~mm}, t=25 \mathrm{~cm}$ und Armierungsstähle $\emptyset 6 \mathrm{~mm}, t=25 \mathrm{~cm}$ in der oberen Lage eingelegt (Bild 7 ). Der entsprechende auf die schlaffe Armierung normierte Armierungsgehalt $\mu_{n}=\left(F_{e}+F_{s} \cdot \sigma_{s f} / \sigma_{e f}\right) / b \cdot h$ betrug $0.34 \%$. Der absolute Armierungsgehalt $\mu=\left(F_{e}+F_{s}\right) / b \cdot h$ ergibt sich $z u 0.19 \%$.

In den Bereichen "kombinierte Beanspruchung", d.h. dort, wo neben der Querbiegung noch Querzug aus der Längsbelastung wirkt, wurden die Beanspruchungen im Anschluss Platte/ Steg jeweils nach den in Abschnitt 1.3 dargestellten Modellen berechnet: Träger $Q 6$ nach "Superposition der Armierungen aus Flanschfachwerkmodell und aus Querbiegung", Träger Q7 nach "Querbiegung mit Querzugkraft aus Flanschfachwerkmodell". Die totale Plattenquerarmierung wurde in diesen Bereichen so bemessen, dass sie gemäss den entsprechenden Modellen unter einer Längsbelastung $P_{f}^{T h}$ von $43 t$ und einer Querbelastung $\bar{P}_{f}^{T h}$ von $2.91 \mathrm{t}$ zum Fliessen kommen sollte. Da die in den Bereichen "reine Querbiegung" eingelegte vorgespante Armierung ( $\square 4 \mathrm{~mm}, t=25 \mathrm{~cm}$ ) in den Bereichen "kombinierte Beanspruchung" als obere Armierung unverändert beibehalten wurde, musste diese dort folgendermassen mit schlaffer Armierung ( $(6 \mathrm{~mm}$ ) ergänzt werden:

Träger Q6:

In der oberen Lage: $\emptyset 6 \mathrm{~mm}, t=8.5 \mathrm{~cm}$ (Bild 7). Dies entspricht einem Fliesskraftverhältnis $\lambda$ von nur noch 0.34 . Der Vorspanrigrad $\kappa^{\prime}$ für ein Verhältnis $\gamma=1.5$ (siehe Abschnitt 1.4.2) ergibt sich zu 0.64 (Tabelle 7). Der auf die schlaffe Armierung normierte Armierungsgehalt $\mu_{n}$ betrug $0.57 \%$, der absolute Armierungsgehalt $\mu$ $0.44 \%$.

In der unteren Lage: $6 \mathrm{~mm}, t=12.5 \mathrm{~cm}$.

Träger Q7:

In der oberen Lage: $\emptyset 6 \mathrm{~mm}, t=8.2 \mathrm{~cm}$ (Bild 7). Fliesskraftverhältnis, Vorspanngrad sowie Armierungsgehalte sind nahezu identisch mit denjenigen des Trägers Q6. In der unteren Lage: Nach Modell keine Armierung notwendig.

\subsubsection{Konstruktive Ergänzungen der Modellarmierungen}

Bei beiden Versuchsträgern $Q 6$ und $Q 7$ wurden die Armierungen sowohl bezüglich Stärke als auch bezüglich Lage genau den entsprechenden Bemessungsmodellen angepasst. In den Bereichen, wo nach Modell keine Armierung notwendig gewesen wäre, wurde oben und unten in der Platte eine konstruktive Minimalarmierung $\emptyset 6 \mathrm{~mm}, t=27.5 \mathrm{~cm}(\mu=$ $0.12 \%$ ) eingelegt. Einen Ueberblick über die nach den Modellen notwendigen und die effektiv eingelegten Armierungen gibt ebenfalls Bild 11. 
Die Versuchsträger Q6 und Q7 wurden wie bereits die Träger Q1 bis Q5 auf dem Aufspannboden der Eidgenössischen Materialprüfungs- und Versuchsanstalt (EMPA) in Dübendorf geprüft. Eine Gesamtansicht zeigt Bild 12. Es wurden zwei Arten von Belastun-

- Die "Längsbelastung" P wurde durch zwei 100 t Pressen in den Drittelspunkten der Spannweite aufgebracht. Damit die Druckkolben der Pressen auch bei zunehmender Trägerkrümmung (Biegung) zwängungsfrei arbeiten konnten, wurde zwischen dem Versuchsträger und den Pressen ein Lastverteilträger verwendet. Um während des Versuchs eine symmetrische und zwängungsfreie Verschiebung der beiden Trägerauflager zu gewährleisten, wurden die dortigen blockierbaren Rollenlager abwechslungsweise gelöst bzw. blockiert.

- Die "Querbelastung" $\bar{P}$ wurde durch 16 Zugkolben ( 15 t) im Abstand von $1=88 \mathrm{~cm}$ aufgebracht, welche an eine zweite unabhängige Pumpanlage angeschlossen waren. Jeder Kolben zieht die Druckplatte mit der Kraft $\bar{P}$ nach unten, wobei die Reaktion dieser Kraft von unten auf den Steg des Trägers abgegeben wird. Die Querbelastungen $\bar{P} b i l-$ den zusammen mit den entsprechenden Reaktionen einen Eigenspannungszustand und verursachen somit in Längsrichtung des Trägers keine zusätzlichen Beanspruchungen und somit auch keine zusätzlichen Längsschubspannungen $\tau_{y x}$. Abgesehen von den versuchstechnischen Vorteilen dieser Belastungsanordnung wird damit auch ein direkter Vergleich zwischen aufgebrachter Belastung $P$ bzw. $\bar{P}$ und Beanspruchung im Anschlussbereich ermöglicht. Um beim Uebergang Platte/Steg längs des Trägers ein möglichst gleichmässiges Querbiegemoment $M_{q}$ zu erzeugen, wurden die Angriffspunkte der Zugkräfte $\bar{P}$ an der Druckplatte durch kleine Lastverteilträger (HEB 100) verdoppelt.

Die Belastungsanordnung ist in Bild 13 dargestellt.

3.2 Versuchsablauf

Bei jeder Laststufe kam das übliche Belastungsschema zur Anwendung, nämlich:

- Verändern der Last innerhalb von zwei Minuten bis zum Erreichen der vorgesehenen Last- bzw. Verformungsgrösse. Ablesen der Anfangslast $P_{A}$ resp. $\bar{P}_{A}$.

- Konstanthalten der Anfangslast $P_{A}$ resp. $\bar{P}_{A}$ während zwei Minuten.

- Konstanthalten einer Deformationsgrösse für die Dauer der Messungen (ca. 60 Min.); entsprechende Reduktion der Last (Relaxation). Nach Beendigung der Messungen Ablesen der Endlast $P_{E}$ resp. $\bar{P}_{E}$.

In der dritten Phase wurde für die Längsbelastung $P$ jeweils die Durchbiegung in Trägermitte und für die Querbelastung $\bar{P}$ die relative Plattendurchbiegung am Trägerende (Bereich "reine Querbiegung") konstant gehalten. 
Es wurden folgende charakteristische Lastgrössen definiert:

$$
\begin{array}{ll}
P_{I}=P_{f}^{T h} / 1.8=24 t, & \bar{P}_{I}=\bar{P}_{f}^{T h} / 1.8=1.64 t \\
P_{I I}=P_{f}^{T h} / 1.3=33 t, & \bar{P}_{I I}=\bar{P}_{f}^{T h} / 1.3=2.28 t \\
P_{I I I}=P_{f}^{T h}=43 t, & \bar{P}_{I I I}=\bar{P}_{f}^{T h}=2.91 \mathrm{t}
\end{array}
$$

Der Belastungsablauf ist in den Bildern 14 und 15 aufgezeichnet. Die Querbelastung $\bar{P}$ wurde jeweils auf eine charakteristische Lastgrösse $\left(\bar{P}_{I}, \bar{P}_{I I}, \bar{P}_{I I I}\right)$ vorgezogen. Anschliessend wurde der Längsbelastung $P$ auf die entsprechende Lastgrösse ( $P_{I}$, $P_{I I}$ und $P_{\text {III }}$ l nachgefahren. Von da an wurde die Querbelastung $\bar{P}$ konstant gehalten bzw. nach jeder Laststufe wieder auf die Anfangslast $\bar{P}_{A}=\bar{P}_{\text {III }}$ erhöht und nur die Längsbelastung $P$ bis zum Bruch weiter vergrössert. In den Diagrammen der Versuchsresultate wird jeweils unterschieden zwischen $P_{u}$ (erstmals erreichte Höchstlast) und $P_{k}$ (Kollapslast, bzw. Ietzte Laststufe vor dem Bruch). Die Lasten der einzelnen Laststufen sind aus den Tabelien 8 und 9 ersichtich.

\subsection{Messungen}

\section{$\underline{3.3 .1 \text { Lasten }}$}

Die in den Trägern wirksamen Schnittkräfte wurden aus den gemessenen Lasten $P$ und $\bar{P}$ ermittelt. Diese konnten jeweils an den Pendelmanometern der zwei voneinander unabhängigen Pumpenanlagen abgelesen werden.

\subsubsection{Durchbiegungen}

Die Stegdurchbiegungen (Bilder 20 und 21) wurden mittels fest installierten induktiven Weggebern im Abstand von 1 Meter zwischen Hallenboden und Träger gemessen (Anzeige in $5 / 1000 \mathrm{~mm})$.

Die Plattendurchbiegungen (Bilder 20 und 21) wurden beim Steg und beim Plattenrand mit einem induktiven Deformeter mit ca. $1.2 \mathrm{~m}$ Basislänge und im Abstand von $1 \mathrm{~m} g e^{-}$ messen (Anzeige in $1 / 100 \mathrm{~mm}$ ).

\subsubsection{Dehnungen. Stauchungen}

Mit einem elektronischen Deformeter von $20 \mathrm{~cm}$ Basislänge (Anzeige in 1/1000 mm) wurde gemessen:

- Die Dehnung der Biegearmierung entlang einer Trägerhälfte (Bilder 22 und 24)

- Die Betonstauchungen der Druckplatte entlang einer Trägerhälfte, sowohl an der Plattenoberseite ( 3 Messreihen) als auch an der Plattenunterseite ( 2 Messreihen) (Bilder 23 und 25).

Mit einem elektronischen Deformeter von $10 \mathrm{~cm}$ Basislänge (Anzeige in 1/1000 mm) wurde gemessen:

- Die Dehnung der schlaffen oberen Plattenquerarmierung an fünf Messstrecken pro Armierungsstab längs der ganzen Platte (Bilder 26 und 27). An der unteren Plattenquerarmierung wurden vereinzelte Messungen durchgeführt (Bilder 26b, 27b). 
freiliegenden Stahl bzw. auf die Betonoberfläche geklebt waren. Mit diesem Verfahren konnten die Messungen bis zum Bruch einwandfrei vorgenommen werden.

Bei der Auswertung der Dehnungsmessungen der Plattenquerarmierung wurde ferner der Einfluṣs der Plattenkrümmung infolge Querbiegung auf die Messwerte berücksichtigt (Bild 28). Die an der oberen resp. unteren Plattenarmierung ermittelten Dehnungen $\varepsilon_{0}$ und $\varepsilon_{u}$ sind nicht in der Mittelinie des Armierungsstabes gemessen, sondern auf der Höhe der Messbolzen. Verschiedene Nachrechnungen für diverse Laststufen bzw. Lastkombinationen haben gezeigt, dass $\varepsilon_{\text {eo }}$ unabhängig von Belastung und Lage des untersuchten Schnittes stets etwa $0.9 \varepsilon_{0}$ beträgt. Zur Bestimmung von $\varepsilon_{\text {eu konnte leider }}$ kein ähnlicher Faktor gefunden werden (untere Armierung zu nahe an Neutralachse). Die Dehnungen $\varepsilon_{e u}$ der unteren Armierungslage mussten jeweils anhand der Dehnungsebene abgeschätzt werden.

Ferner wurde für die Umrechnung der Dehnungen auf Spannungen folgender Effekt berücksichtigt: Mit einem Deformeter wird eine Längenänderung $\Delta l$ der Basislänge $l_{0}$ gemessen. Bildet man den Quotienten $\Delta 1 / 1_{0}$, so erhält man eine mittlere Dehnung $\varepsilon_{m}$ über diese Basislänge. Vor allem beim Stahl ergeben sich aber im gerissenen Stadium Abweichungen zwischen einer mittleren Dehnung $\varepsilon_{e m}$ und der maximalen Dehnung $\varepsilon_{e} \max$ im Riss. Für die Umsetzung der mittleren Dehnungen $\varepsilon_{e m}$ in die Spannungen resp. Kräfte im Rissequerschnitt (anhand des $\sigma-\varepsilon$-Diagramms) wurde ein Koeffizient $k=\varepsilon_{e m} / \varepsilon_{e} \max$ von 0.9 verwendet. Eine Begründung für diese Annahme wird aufgrund entsprechender Versuche in [1] gegeben.

\subsubsection{Risse}

Nach jeder Laststufe wurden die in der Platte von blossem Auge sichtbaren Risse mit Filzstift nachgezogen und die Rissbreite mit einem Rissemassstab ausgemessen (Einschätzung auf $0.05 \mathrm{~mm}$ ). Die Rissbreiten wurden auf Klebern notiert und diese neben den Rissen fixiert. Anschliessend wurden das Rissebild und damit auch die Rissbreiten photographisch festgehalten (z.B. Bilder 16 bis 19). 
In diesem Kapitel werden die Resultate der beiden geprüften Träger wiedergegeben. Beide Versuchsträger, Q6 und $Q 7$, wurden sowohl einer Längsbelastung $P$ als auch einer Querbelastung $\bar{P}$ unterworfen, d.h. die teilweise quervorgespannte Druckplatte wurde im Anschlussbereich Platte/Steg durch Längsschub $\tau_{x y}$ und Querbiegung $M_{q}$ beansprucht. Zur Erzeugung des Längsschubes wurden die Träger durch zwei Einzellasten in den Drittelspunkten der Spannweite belastet. Das Querbiegemoment wurde durch 16 Zugkolben sozusagen als Eigenspannungszustand aufgebracht (Bild 13). Die Druckplatte des Versuchträgers Q6 war nach dem Modell "Superposition der Armierungen aus Flanschfachwerkmodell und aus Querbiegung" und diejenige des Versuchsträgers Q7 nach dem Modell

"Armierung für Querbiegung mit Querzugkraft aus Flanschfachwerkmodell" bemessen (Bild5).

Für die Darstellung der Versuchsresultate wurden folgende charakteristische Lastgrössen bzw. Lastkombinationen gewählt:

- Gebrauchslast der Plattenquerarmierung (1. Stufe)

$$
P_{I}=\frac{P_{I I I}}{1.8}=24 t, \quad \bar{P}_{I}=\frac{\bar{P}_{I I I}}{1.8}=1.64 t
$$

- Laststufe zwischen Gebrauchs- und Fliesslast der Plattenquerarmierung (2. Stufe)

$$
P_{I I}=\frac{P_{I I I}}{1.3}=33 \mathrm{t}, \quad \bar{P}_{I I}=\frac{\bar{P}_{I I I}}{1.3}=2.28 \mathrm{t}
$$

- Theoretische Fliesslast der Plattenquerarmierung (3. Stufe)

$$
P_{I I I}=P_{f}^{T h}=43 \mathrm{t}, \quad \bar{P}_{I I I}=\bar{P}_{f}^{T h}=2.91 \mathrm{t}
$$

- Höchstlast der Versuchsträger

$$
P_{u}=P_{u A}^{E x} \quad=56 \mathrm{t}, \quad \bar{P}_{f}^{T h} \cong P_{u A}^{T h}=2.91 \mathrm{t}
$$

- Kollapslast der Versuchsträger (letzte gemessene Laststufe)

$$
P_{k}=P_{U A}^{E x} \quad=56 \mathrm{t} . \quad \quad \bar{P}_{f}^{T h} \cong \bar{P}_{U A}^{T h}=2.91 \mathrm{t}
$$

\subsection{Allgemeines Tragverhalten}

Die ersten Risse in der Druckplatte erschienen bei beiden Trägern beim Steigern der Querbelastung $\bar{P}$ von $\bar{P}_{I}=1.61 \mathrm{t}$ auf $\bar{P}_{I I}=2.28 \mathrm{t}$ (Bilder 32 und 33) und zwar fast längs der ganzen Druckplatte im Anschlussschnitt Platte/Steg. Die gleichzeitig wirkende Längsbelastung $P$ betrug $P_{I}=24$ t. Da die ersten Risse erst gegen Ende des Belastungsvorganges in Erscheinung traten, wurde die Risslast der Laststufe $\bar{P}_{I I}=2.28 t$, $P_{I}=24 t$ zugeordnet. Während der theoretische Vorspanngrad $k$ ', berechnet mit einer zulässigen Randzugspannung von $10 \mathrm{~kg} / \mathrm{cm}^{2}$ nach Norm SIA 162 , weniger als 1.0 betrug (Tabelle 7), traten die ersten Risse erst etwa bei einem "effektiven"Vorspanngrad $\kappa^{\prime}$ von 1.3 auf. Der Grund liegt darin, dass in den Versuchen eine wesentlich höhere Randzugfestigkeit des Betons vorhanden war. Mit den Beanspruchungen gemäss den Bemessungsmodellen ergeben sich rund $30 \mathrm{~kg} / \mathrm{cm}^{2}$ im Bereich "reine Querbiegung und rund $40 \mathrm{~kg} / \mathrm{cm}^{2}$ im Bereich "kombinierte Beanspruchung". 
Die Plattenquerarmierung kam erst etwa bei der Höchstlast $\bar{P}_{\text {III }}=2.91 \mathrm{t}, \mathrm{P}_{U}=56 t$ im Bereich "kombinierte Beanspruchung" an mehreren Stellen ins Fliessen (Bilder 26 und 27). Die theoretische Fliesslast $\vec{P}_{f}^{T h}=2.91 \mathrm{t}, P_{f}^{T h}=43 \mathrm{t}$ (Tabelle 10) wurde damit wesentlich überschritten. Das einfache Flanschfachwerkmodell mit dem Ausbreitwinkel $\beta=\operatorname{arctg} 0.5$ und der entsprechenden Querzugkraft pro Längeneinheit von

$$
z=\frac{Q}{4 \cdot y}
$$

überschätzt die tatsächliche Beanspruchung des Anschlusses Platte/Steg. Durch die Berücksichtigung des Einflusses der Stegbreite bo auf die Querzugkraft, d.h. mit

$$
z=\frac{Q}{4 \cdot y} \cdot \frac{b-b_{0}}{b}
$$

kann jedoch eine gute Uebereinstimmung der theoretischen mit der experimentellen Fliesslast erreicht werden (siehe Abschnitt 5.1).

Beide Versuchsträger verhielten sich, wie die Last-Durchbiegungs-Diagramme (Bilder 14 und 15) zeigen, bis zum Bruch nahezu elastisch-plastisch. Nach Erreichen der Höchstlast $P_{U A}^{E x}=56$ t kam die Längsarmierung ins Fliessen, und bis zur Kollapslast $P_{k}=56 t$ (letzte Laststufe vor dem Bruch) konnte kein Lastabfall festgestellt werden. Der Bruch der Versuchsträger erfalgte als reiner Biegebruch durch Versagen der Betondruckzone (Bilder 17 und 19). Der Unterschied der gemessenen Bruchlast $P_{u A}^{E x}=$ $56 t$ und der nachberechneten Bruchlast $P_{U A}^{T h}=60 t$ (Tabelle 10) lässt sich auf das nicht berücksichtigte Eigengewicht der Träger und Versuchseinrichtungen sowie auf die gegenüber den Berechnungsannahmen effektiv etwas kleinere statische Höhe der eingelegten Längsarmierung zurückführen.

\subsection{Biegeverhalten der Träger}

\subsubsection{Durchbiegungen}

Die Stegdurchbiegungen der Versuchsträger $\delta_{s}$ sind in den Bildern 20 und 21 dargestellt. Sie erreichten in Trägermitte beim Fliessbeginn der Längsarmierung ( $\bar{P}_{\text {III }}=2.91$ t, $P_{u}=56 \mathrm{t}$ ) etwa $4 \mathrm{~cm}$, um dann noch bis auf etwa $7 \mathrm{~cm}$ bei der letzten Laststufe vor dem Bruch $\left(\bar{P}_{I I I}=2.91 \mathrm{t}, P_{k}=56 t\right)$ anzuwachsen.

Die relativen Durchbiegungen $\delta_{r}$ der Plattenränder längs der Träger sind in den gleichen Bildern aufgezeichnet. Sie wurden hauptsächlich durch die Querbelastung $\bar{P}$ hervorgerufen und erreichten schliesslich etwa $2 \mathrm{~mm}$. Die Durchbiegungen $\delta_{\Gamma}$ verlaufen etwa proportional zu den Dehnungen der oberen Plattenquerarmierung (Bilder 26a und $27 a$ ).

\subsubsection{Dehnungen der Läng sarmierung}

Die mittleren Dehnungen der Längsarmierung (untere Lage) sind in den Bildern 22 und 24 dargestellt. Da hier mittlere Dehnungen der $20 \mathrm{~cm}$ langen Messstrecken aufgetragen sind, wird mit $k \cdot \varepsilon_{\text {ef }}$ versucht, den Fliessbeginn zu erfassen $(k \cong 0.9)$.

\section{2 .3 Stauchungen der Qruckplatte}

Die auf einer Trägerhälfte gemessenen Betonstauchungen gehen aus den Bildern 23 und 25 hervor. Weil sich auf der Plattenober- bzw. Plattenunterseite die Stauchungen der verschiedenen Messreihen kaum voneinander unterschieden, wurde jeweils das Mittel der oberen bzw. unteren Messreihen aufgetragen. 
Die für das Ziel der Untersuchung besonders wichtigen Dehnungen der Plattenquerarmierung der Versuchsträger Q6 und Q7 sind in den Bildern 26 und 27 dargestellt. Durch $\kappa \cdot \varepsilon_{\text {ef }}$ wird stets der Fliessbeginn angedeutet.

Jeweils die grösste Dehnung aus den fünf Messstrecken eines oberen schlaffen Armierungsstabes wurde in den Bildern 26a und 27a aufgezeichnet. Die ersten zwei dargestellten Laststufen weisen die gleiche Querbelastung $\bar{P}_{I I}=2.28 t$ sowie die Längsbelastung $P_{I}=24 \mathrm{t}$ bzw. $P_{I I}=33 \mathrm{t}$ auf. Die Laststufe $\bar{P}_{I I}=2.28 \mathrm{t}, P_{I}=24 t$ entspricht der ersten Laststufe nach Beginn der Rissebildung. Die andern vier dargesteliten Laststufen weisen die gleiche Querbelastung $\overline{\mathrm{P}}_{\text {III }}=2.91 \mathrm{t}$ auf, wobei die Längsbelastung $P$ von $P_{I I}=33 t$ auf $P_{k}=56 t$ gesteigert wurde.

Die Bilder 26b und 27b zeigen die Querverteilung der Stahldehnungen in vier reprësentativen Schnitten $A$ bis $D$. Die Schnitte $A$ und $B$ befinden sich im Bereich "kombinierte Beanspruchung", Schnitt $C$ in Trägermitte und Schnitt $D$ nach dem Flanschfachwerkmodell im Bereich "reine Querbiegung". Die Dehnungen der unteren Plattenquerarmierung sind nur bei der theoretischen Fliesslast $\left(\bar{P}_{\text {III }}=2.91 \mathrm{t}, P_{\text {III }}=43 \mathrm{t}\right)$ punktweise aufgetragen. Sie weisen sehr geringe Werte auf. Ausser der ersten Kurve ( $\bar{P}_{I I}=2.28 t$, $\left.P_{I I}=33 \mathrm{t}\right)$ gehören die anderen drei Kurven zur gleichen Querbelastung $\bar{P}_{\text {III }}=2.91 \mathrm{t}$, sie entsprechen jedoch unterschiedlichen Längsbelastungen $P_{I I I}$ bis $P_{k}$. Somit wird der Zusammenhang zwischen Dehnungszuwachs und massgebender Belastung direkt ersichtlich. In den Schnitten $A, B$ und $C$ ist eine Zunahme der Dehnungen infolge Steigerung der Längsbelastung von $P_{I I I}=43 t$ auf $P_{k}=56 t z u$ sehen. Im Schnitt $D$ hingegen bewirkt eine Zunahme der Längsbelastung $P$ fast keine Zunahme der Dehnungen. Mit Ausnahme des Schnittes C (Trägermitte), wo der Dehnungszuwachs vor allem auf die "Verträglichkeitswirkung" der Druckplattenhälften (Scheiben) zurückzuführen ist, stehen die Dehnungen der Querarmierung in gutem Einklang mit den Modellen (vgl. Abschnitt 5.1). Die geringen Dehnungen in der mittleren Messstrecke direkt über dem Steg, im Vergleich zu den benachbarten Dehnungen im Anschlusschitt Platte/Steg, lassen sich vor allem durch die Abnahme der Armierungszugkraft infolge Querbiegung (Vergrösserung Biegehebelarm) erklären.

Die Bilder $26 \mathrm{c}$ und $27 \mathrm{c}$ stellen den Last-Dehnungsverlauf der in den Schnitten $A$ bis $\square$ eingetragenen vier Messstellen dar. Auch hier kann man feststellen, dass bei Messstelle 4 (Schnitt D) die Dehnungen fast nur von der Querbelastung beeinflusst werden.

Ein Unterschied der Dehnungen der Plattenquerarmierung zwischen den Versuchsträgern Q6 und $Q 7$ lässt sich bis zur Höchstlast $\left(\bar{P}_{\text {III }}=2.91 t, P_{u}=56 t\right)$ kaum feststellen. Erst bei der Ietzten Laststufe $\left(\bar{P}_{\text {III }}=2.91 \mathrm{t}, P_{k}=56 \mathrm{t}\right)$ weist der Versuchsträger Q7 in Trägermitte grössere Dehnungen (Bilder 26a und 27a) auf. 
Der Verlauf der Kräfte in der oberen Plattenquerarmierung längs der Träger ist in den Bildern 29 und 30 dargestelt. Die Kräfte beziehen sich auf die maximalen Stahldehnungen $\varepsilon_{e} \max$ im Rissequerschnitt $\left(\varepsilon_{e \max }=\varepsilon_{e m} / K, \sigma_{e} \max\right.$ aus $\varepsilon_{e} \max$ im $\sigma-\varepsilon-D i a-$ gramm, siehe Abschnitt 3.3.3). Zwischen den Versuchsträgern Q6 und Q7 ist kein wesentlicher Unterschied im Kräfteverlauf der oberen Plattenquerarmierung festzustellen. Nebst der Vorspannkraft $V=5.9 \mathrm{t} / \mathrm{m}$ und der Resultate der Laststufe $\bar{P}_{\text {II }}=2.28 \mathrm{t}$, $\mathrm{P}_{I I}=33 \mathrm{t}$, wurden die Resultate von vier Laststufen gleicher Querbelastung $\bar{P}_{\text {III }}=$ $2.91 \mathrm{t}$ und steigender Längsbelastung (von $P_{I I}=33 \mathrm{t}$ auf $P_{k}=56 \mathrm{t}$ ) aufgetragen.

\subsection{Risseverhalten}

Die in Querrichtung teilweise vorgespannte Druckplatte verhielt sich bei beiden Trägern bis über die Gebrauchslast hinaus rissefrei. Die ersten Risse entstanden beim Steigern der Querbelastung von $\bar{P}_{I}=1.64 \mathrm{t}$ auf $\bar{P}_{I I}=2.28 \mathrm{t}$. Die gleichzeitig wirkende Längsbelastung betrug $P_{I}=24$ t. Im Bereich "reine Querbiegung" entspricht dies einem effektiven Vorspanngrad $K^{\prime}$ (siehe Abschnitt 1.4.2) von etwa 1.3 und damit einer Randzugfestigkeit des Betons von etwa $30 \mathrm{~kg} / \mathrm{cm}^{2}$. Im Bereich "kombinierte Beanspruchung" ergibt sich gemäss den verwendeten Bemessungsmodellen für das vorliegende Verhältnis $\gamma=\frac{Z}{M_{q} / d} \cong 1.5$ eine Randzugfestigkeit des Betons von etwa $40 \mathrm{~kg} / \mathrm{cm}^{2}$ (Tab. 7). Die Rissebilder bei der Höchstlast $\left(\bar{P}_{\text {III }}=2.91 \mathrm{t}, \mathrm{P}_{U}=56 \mathrm{t}\right)$ sind für beide Träger in Bild 31 dargestellt. Die Entstehung dieser Rissebilder in der linken Trägerhälfte ist aus den Bildern 16 und 18 ersichtlich.

Die maximalen Rissweiten längs der Träger sind in den Bildern 32 und 33 aufgezeichnet (Einschätzung auf $0.05 \mathrm{~mm}$ ). Im Bereich "reine Querbiegung" erreichen bei beiden Trägern die Risse vor dem Bruch eine Breite von etwa $0.55 \mathrm{~mm}$. Im Bereich "kombinierte Beanspruchung" dagegen sind es beim Träger Q6 etwa $0.35 \mathrm{~mm}$ und beim Träger Q7 etwa 0.25 $\mathrm{mm}$. Auf den gleichen Bildern ist ferner in Funktion der Last die Breite des jeweils grössten Risses (ganze Platte) dargestellt. Dieser Riss wurde stets im Bereich "reine Querbiegung" festgestellt. 
5. VERGLEICHE

5.1 Vergleich der Ergebnisse mit den Modellen

Aufgrund der durch die Quervorspannung bewirkten sehr späten Rissebildung werden in Bild 34 die gemessenen Zugkräfte der oberen Plattenquerarmierung nur bei der theoretischen Fliesslast $\left(\bar{P}_{f}^{T h}=2.91 \mathrm{t}, \mathrm{P}_{f}^{T h}=43 \mathrm{t}\right)$ mit den entsprechenden Eeanspruchungen aus den Modellen bzw. mit den Fliesswiderständen der obern Armierungslage verglichen.

Bei beiden Versuchsträgern, Q6 und Q7, ist festzustellen, dass im Bereich "kombinierte Beanspruchung" die gemessenen Zugkräfte kleiner sind als nach den Modellen zu erwarten war. Im Bereich "reine Querbiegung" hingegen stimmen gemessene und nach Theorie zu erwartende Zugkräfte gut überein. Dies weist darauf hin, dass das einfache Flanschfachwerkmodell mit dem Ausbreitwinkel $\beta=\operatorname{arctg} 0.5$ und der entsprechenden Querzugkraft pro Längeneinheit von $Z=\frac{Q}{4 \cdot y}$ die tatsächlichen Beanspruchungen überschätzt.

Eine bessere Uebereinstimmung kann erreicht werden, indem im Flanschfachwerkmodell bei gleichem Ausbreitwinkel $\beta$ der Einfluss der Stegbreite b berücksichtigt wird (siehe Abschnitt 1.3). Dadurch erhält man eine verringerte Querzugkraft pro Längeneinheit von $z=\frac{Q}{4 y} \cdot \frac{b-b_{0}}{b}$. Mit diesem Modell ergibt sich eine höhere theoretische Fliesslast $\left(\bar{P}_{f}^{T h}=2.91 \mathrm{t}, P_{f, \text { neu }}^{T h}=54 \mathrm{t}\right)$ für die Plattenquerarmierung. Bei dieser neuen Fliesslast wurden die in Bild 35 dargestellten Kräfte gemessen. Sie weisen eine erheblich bessere Uebereinstimmung mit den Fliesswiderständen der eingelegten Armierungen auf. In verschiedenen Schnitten wird der fliesswiderstand erreicht. In Tabelle 11 ist das Verhältnis der gemessenen zu den theoretischen Zugkräften im Bereich "kombinierte Beanspruchung" angegeben. Dabei werden nur die Zugkräfte infolge Längsschub miteinander verglichen, d.h. sowohl bei den gemessenen Werten $k_{\text {tot }}^{E x w}$. $K_{\text {tot, neu }}^{\text {Ex }}$ als auch bei den theoretischen Werten $K_{\text {tot }}^{T h}$ wurde die berechnete Zugkraft infolge reiner Querbiegung $K_{M_{G}}^{T h}$ jeweils abgezogen. Bei beiden Versuchsträgern erreichten im Beraich "kombinierte Beanspruchung" die Spitzenwerte der gemessenen Zugkräfte den Fliesswiderstand an etwa der gleichen Stelle, nämlich am Ende dieses Bereiches gegen die Trägermitte zu (Bild 35). Die Mittelwerte über den Bereich "kombinierte Beanspruchung" hingegen liegen etwas tiefer (Tabelle 11).

Daraus lassen sich die folgenden Schlüsse ziehen:

1. Für das Verformungs- und Tragverhalten der Versuchsträger ist es vorteilhaft, den Bemessungsbereich für Längsschub vor allem gegen die Trägermitte hin zu verbreitern (siehe Abschnitt 1.3). Dadurch werden die Dehnungs-bzw. Kraftspitzen abgebaut und das Verformungs- und Tragverhalten verbessert.

2. Für die anhand der knapp $20 \%$ tiefer liegenden Mittelwerte (für die Gesamtbeanspruchung ink1. Querbiegung sind es nur rund 10\%) feststellbare Tragreserve können die folgenden beiden Erklärungen angeführt werden:

a) Der "tatsächliche" Ausbreitwinkel im Flanschfachwerk ist etwas kleiner als $\beta=\operatorname{arctg} 0.5$, beispielsweise $\beta=\operatorname{arctg} 0.4$.

b) Die Querzugkraft $Z$ aus Längsschub verlagert sich aus der Plattenmitte etwas gegen die Biegedruckzone hin. Dadurch wird die obere Längsarmierung entlastet. 
Es ist denkbar, dass nur die eine oder die andere dieser Wirkungen eintritt, oder dass beide je einen Teil zur Tragreserve beitragen.

5.2 Vergleich der Ergebnisse der Versuche mit und ohne Quervorspannung

Die nur schlaff armierten Versuchsträger Q4 bzw. Q5 [1] entsprachen bezüglich den für die Bemessung der Druckplatte verwendeten Modellen den teilweise quervorgespannten Versuchsträgern Q6 bzw. Q7. Bei Q4 und Q6 bzw. bei Q5 und Q7 wies die obere Querarmierung den gleichen Fliesswiderstand auf. Bei $Q 6$ und $Q 7$ bestand jedoch längs des ganzen Trägers ein Teil def Armierung ( $\emptyset 4 \mathrm{~mm}, t=25 \mathrm{~cm}$ ) aus normal vorgespanntem Spannstahl. Ueber die bei diesen Trägern in den Bereichen "reine Querbiegung" und "kombinierte Beanspruchung" vorhandenen Vorspanngrade $k$ ', gerechnet mit den nach Norm SIA 162 zulässigen Randzugspannungen von $10 \mathrm{~kg} / \mathrm{cm}^{2}$, gibt die dritte Spalte von Tabelle 7 Auskunft.

Hier sollen insbesondere die Träger $Q 4$ und $Q 6$ miteinander verglichen werden. Ein Vergleich der Träger $Q 5$ und $Q 7$ zeigt praktisch dieselben Ergebnisse.

\subsubsection{Anrisslast der Druckplatte}

Beim schlaff armierten Versuchsträger Q4 traten die ersten Risse in der Druckplatte beim Aufbringen der Querbelastung $\bar{P}_{I}=1.64 \mathrm{t}$ auf. Die Anrisslast lag also tiefer als die Gebrauchslast $\left(\bar{P}_{I}=\bar{P}_{f}^{T h} / 1.8\right)$.

Beim teilweise quervorgespannten Versuchsträger Q6 hingegen konnten die ersten Risse erst beim Steigern der Querbelastung $\bar{P}$ von $\bar{P}_{I}=1.64 t$ auf $\bar{P}_{I I}=2.28$ f festgestelit werden. Die Anrisslast lag somit höher als die Gebrauchslast.

Die teilweise Quervorspannung der Druckplatte hat, wie zu erwarten war, einen grossen Einfluss auf die Anrisslast. Es ist jedoch zu beachten, dass bei den vorliegenden Versuchen die ersten Risse erst etwa bei den folgenden rechnerischen Betonspannungen am Zugrand $\sigma_{\Gamma}$ festgestellt wurden:

$$
\begin{array}{ll}
\text { Bereich "reine Querbiegung" } & \text { ca. } 30 \mathrm{~kg} / \mathrm{cm}^{2} \\
\text { Bereich "kombinierte Beanspruchung" : ca. } 40 \mathrm{~kg} / \mathrm{cm}^{2}
\end{array}
$$

und zwar sowohl bei den schleff armierten als auch bei den teilweise quervorgespannten Varsuchsträgern.

Bei einem konkreten Bauwerk dürften z.B. infolge Schwind- und Temperaturzwängungen, oft wiederholter Belastungen usw. nicht derartig hohe Werte auftreten. Trotzdem hat die teilweise Vorspannung (nach Norm SIA 162 muss für ständige Lasten volle Vorspannung, definiert mit einer zulässigen Randzugspannung für hochwertigen Beton BH von $10 \mathrm{~kg} / \mathrm{cm}^{2}$ vorhanden sein) einen wichtigen Vorteil: Im Bereich der Gebrauchslasten treten bei teilweise vorgespannten Bauteilen, sofern sie mit schlaffer Armierung fachgerecht ausarmiert sind noch keine oder jedenfalls dünnere Risse als bei analogen, nur schlaff armierten Bauteilen auf. 


\subsubsection{Risseverhalten}

In Bild 36 werden anhand der Versuchsträger Q4 und Q6 die unterschiedlichen Rissebilder einer schlaff armierten und einer analogen teilweise quervorgespannten Druckplatte einander gegenübergestellt. Bei der schlaff armierten Druckplatte von Q4 sind etwa vier bis sechs, bei der teilweise quervorgespannten Druckplatte von Q6 dagegen nur etwa zwei bis vier Risslinien, mit entsprechend grösseren Rissabständen, festzustellen.

In Bild 37 wird ein Vergleich der maximalen Rissbreiten längs der Plattenoberseite der Träger Q4 und Q6 dargestelit. Ein Vergleich der Träger Q5 und Q7 würde ein ähnliches Bild ergeben.

In den Bildern $38 a$ und $38 b$ wird der unterschiedliche Verlauf der maximalen Rissbreiten in Abhängigkeit der Last zwischen dan Trägern $Q 4$ und $Q 6$ bz:w. Q5 und $Q 7$ dargestellt. Aufgrund der bedeutend späteren Rissebildung bei der teilweise vorgespannten Platte verhält sich diese bis $\bar{P}_{I I}$, $P_{I I}$, d.h. bis zur etwa 1.4-fachen Gebrauchslast günstiger als die nur schlaff armierte Platte. Oberhalb dieser Last - was für die Praxis allerdings belanglos ist - wurden dann aber in den Druckplatten der Versuchsträger $Q 6$ und Q7 breitere Risse als bei den entsprechenden schlaff armierten Versuchsträgern $Q 4$ und $Q 5$ festgestellt. Dies ist vor allem auf den grösseren Risseabstand in der vorgespanten Platte zurückzuführen.

\subsubsection{Dehnungen der Plattenquerarmierung}

Nachdem die Anrisslast der Druckplatte überschritten war, glichen sich die Stahldehnungen der teilweise quervorgespannten Träger Q6 bzw. Q7 denjenigen der nur schlaff armierten Träger Q4 bzw. Q5 an.

In Bild 39 wird ein Vergleich der maximalen Stahldehnungen der oberen Plattenquerarmierung der Träger Q4 und Q6 gezeigt. Ein Vergleich zwischen den Trägern Q5 und Q7 würde ein ähnliches Bild ergeben.

In den Bildern 40a und 40b wird bei der Messstelle 1 (Bereich "kombinierte Beanspruchung") der unterschiedliche Verlauf der Stahldehnungen in Abhängigkeit der Last bei den Trägern Q4 und Q6 bzw. Q5 und Q7 dargestellt. Von der Laststufe $\bar{P}_{I I I}=2.91 \mathrm{t}$, $P_{I I}=33 t$ ( 2 Laststufen nach der Risslast) an ist zwischen den teilweise quervorgespannten und den nur schlaff armierten Versuchsträgern kein wesentlicher Unterschied in den Stahldehnungen mehr festzustellen.

\subsubsection{Kräfte in der Plattenquerarmierung}

Der Kräfteverlauf in der oberen Armierungslage für $\bar{P}_{\text {III }}=2.91 \mathrm{t}$, $P_{\text {III }}=43 \mathrm{t}$ und $\bar{P}_{I I I}=2.91 \mathrm{t}, \mathrm{P}_{U}=56 \mathrm{t}$ wurde in Bild 41 für die Träger Q4 und Q6 aufgezeichnet. Es ist deutlich ersichtlich, dass bei hohen Laststufen die Quervorspannung der Druckplatte keinen Einfluss auf die Beanspruchung der Plattenquerarmierung mehr hat. Dies bedeutet, dass das Flanschfachwerkmodell seine Gültigkeit behält ungeachtet, ob die Druckplatte nur schlaff armiert oder teilweise quervorgespannt ist. 
Der entscheidende Vorteil einer teilweisen Quervorspannung der Druckplatte gegenüber einer nur schlaffen Armierung für "Längsschub mit Querbiegung" wie auch für "Querbiegung allein" ergibt sich durch das bedeutend günstigere Risseverhalten im Gebrauchslastbereich. Risse treten erst bei höheren Lasten auf, und die Rissbreiten waren bei den vorliegenden Versuchen in den vorgespanten Druckplatten bis zur 1.4-fachen Gebrauchslast kleiner als in den analogen nur schlaff armierten Platten. Im Bereich der rechnerischen Bruchlast hat die Quervorspannung keinen Einfluss mehr auf die Beanspruchung der Plattenquerarmierung.

Für die Bemessung von in Querrichtung teilweise vorgespannten Druckplatten von Betonträgern für Längsschub und Querbiegung können folgende vorläufige Empfehlungen gegeben werden.

\section{Bemessung auf Tragfähigkeit}

Die erforderliche Fliesskapazität der Gesamtarmierung (vorgespannte und schlaffe Armierung) kann gleich wie bei nur schlaff armierten Druckplatten berechnet werden ( $v g l$. [1]]:

a) Die pro Längeneinheit des Trägers infolge Längsschub erforderliche Fliesskapazität der Gesamtarmierung (vorgespante und schlaffe Armierung) kann nach der Formel

$$
z=\frac{Q}{5 \cdot y} \cdot \frac{b-b_{0}}{b} \quad b z w \cdot \quad z=\frac{Q}{4 \cdot y} \cdot \frac{b-b_{0}}{b}
$$

bemessen werden, je nachdem, ob ein Fliessen einzelner Armierungsstäbe vor Erreichen der Biegekapazität bzw. der Stegschubkapazität des Trägers in Kauf genommen wird oder nicht.

b) Die Armierung ist gegenüber dem Trägerquerschnitt mit der Querkraft $Q$ um das Versatzmass

$$
v \cong 0.6(y+b)
$$

in Richtung des zunehmenden Momentes zu verschieben.

c) Die Längsschubarmierung kann im allgemeinen je zur Hälfte oben und unten in die Platte eingelegt und der Querbiegearmierung überlagert werden. Auf der Biegedruckseite der Platte kann die Längsschubarmierung entsprechend der gleichzeitig wirkenden Biegedruckkraft abgemindert werden.

d) Längs des ganzen Trägers ist in der Druckplatte je oben und unten eine konstruktive Mindestarmierung von 0.10 bis $0.15 \%$ einzulegen.

e) Die obere Schubspannungsgrenze ist auf rund 70 bis $80 \%$ des Wertes ohne Querbiegung abzumindern. 


\section{Bemessung für den Gebrauchszustand (Rissesicherung)}

f) Der Vorspanngrad für die Beanspruchung "reine Querbiegung" und "Querbiegung mit Querzugkraft" wird mit

$$
k^{\prime}=\frac{M_{z u 1, v}}{M_{g+p}}
$$

definiert. Der Einfluss der Querzugkraft kann durch das Verhältnis $\gamma=Z \cdot d / M$ erfasst werden. Bei gegebenem $\gamma$ ist $M_{z U l, v}$ das Moment, welches am ungerissenen Querschnitt die Randzugspannung $\sigma_{r}$ erzeugt. M $g_{+p}$ ist das Moment unter voller Gebrauchslast. Bei gleichbleibender Vorspannung bewirkt $\gamma$ eine Abnahme des Vorspanngrades (Tabelle 7 ).

g) Der Vorspanngrad kann nach wirtschaftlichen Gesichtspunkten festgelegt werden, wobei

- die Begrenzung nach unten im allgemeinen durch die Bedingung $k^{\prime} \geq M_{g} / M_{g+p}$ erfolgt (volle Vorspannung für ständige Lasten),

- Die Begrenzung nach oben bedingt ist durch die Notwendigkeit, auch in vorgespannten Konstruktionen stets eine schlaffe Armierung anzuordnen.

h) Für ein gutes Risseverhalten ist die schlaffe Armierung in Form von eher vielen dünnen Stäben einzulegen.

\section{Einschränkungen}

Die obigen Empfehlungen sind als vorläufig zu betrachten. Sie gelten nur für bezüglich Geometrie und Belastung symmetrische T-Querschnitte. Modelle und Empfehlungen für andere Fälle befinden sich in Vorbereitung.

Die Empfehlungen sind ausdrücklich nur für Druckplatten gültig. In Zugplatten ist eine stärkere Längsschubarmierung erforderlich. Hierüber wird in einer kommenden Publikation berichtet. 
In [1] ist über Versuche an fünf Stahlbetonträgern mit Plattenbalkenquerschnitt berichtet worden. Die Druckplatten waren bei zwei Trägern [Q1, Q2) durch "Längsschub allein" und bei drei Trägern (Q3, Q4, Q5) durch "Längsschub mit Querbiegung" beansprucht. Im vorliegenden Bericht werden die Ergebnisse von Versuchen an zwei weiteren Trägern (Q6, Q7), deren Druckplatten in Querrichtung vorgespannt waren, mitgeteilt. Anhand dieser Träger sollte der Einfluss einer teilweisen Vorspannung für Längsschub und Querbiegung näher abgeklärt werden (Bild 12). Insbesondere interessierte der Vergleich zwischen analogen Trägern mit und ohne Quervorspannung.

Da sich bei der in [1] beschriebenen Versuchsserie das Flanschfachwerkmodell als zweckmässig und der herkömmlichen Methode mit der Aufnahme von Hauptzugsspannungen überlegen erwiesen hatte, wurde hier nur das Flanschfachwerkmodell verwendet. Die total erforderliche Plattenquerarmierung (vorgespannte und schlaffe Armierung) der Träger Q6 und Q7 wurde aufgrund einer plastischen Bemessung analog den früher geprüften Trägern Q4 und Q5 bestimmt :

Träger Q6: Nach "Superposition der Armierungen aus Flanschfachwerkmodell und aus Querbiegung" (Bild 5a).

Träger Q7: Nach "Armierung für Querbiegung mit Querzugkraft aus Flanschfachwerkmodell" (Bild 5b).

Die Quervorspannung der Druckplatten wurde längs der Träger unverändert durchgeführt und so gewählt, dass der mit einer zulässigen Randzugspannung von $10 \mathrm{~kg} / \mathrm{cm}^{2}$ berechnete Vorspanngrad $k^{\prime}$ in den Plattenbereichen mit reiner Querbiegung den Wert 0.80 und in den Bereichen mit Querbiegung und Querzugkraft aus Längsschub etwa den Wert 0.64 annahm (Tabelle 7 ).

Alle übrigen Grössen wie Trägerabmessungen, Biege- und Querkraftarmierung des Steges, Betonqualität, usw., wurden von den Trägern Q1 bis Q5 unverändert übernommen (Bild 6).

Der entscheidende Vorteil einer teilweisen Quervorspannung der Druckplatte gegenüber einer nur schlaffen Armierung für "Längsschub mit Querbiegung" wie auch für "Querbiegung allein" ergibt sich durch das bedeutend günstigere Risseverhalten im Gebrauchslastbereich. Risse treten erst bei höheren Lasten auf, und die Rissbreiten waren bei den vorliegenden Versuchen in den vorgespannten Druckplatten bis zur 1.4-fachen Gebrauchslast kleiner als in den analogen nur schlaff armierten Platten. Im Bereich der rechnerischen Bruchlast hat die Quervorspannung keinen Einfluss mehr auf die Beanspruchung der Plattenquerarmierung.

Die Druckplatten sind sowohl auf Tragfähigkeit als auch auf ein genügendes Risseverhalten im Gebrauchszustand zu bemessen. Aus den Versuchsresultaten können die nachfolgenden vorläufigen Empfehlungen abgeleitet werden.

\section{Bemessung auf Tragfähigkeit}

a) Die pro Längeneinheit des Trägers infolge Längsschub erforderliche Fliesskapazität der Gesamtarmierung (vorgespannte und schlaffe Armierung) kann nach der Formel 


$$
z=\frac{Q}{5 \cdot y} \cdot \frac{b-b_{a}}{b} \quad b z w \cdot \quad z=\frac{Q}{4 \cdot y} \cdot \frac{b-b}{b}
$$

bemessen werden, je nachdem, ob ein Fliessen einzelner Armierungsstäbe vor Erreichen der Biegekapazität bzw. der Stegschubkapazität des Trägers in Kauf genommen wird oder nicht.

b) Die Armierung ist gegenüber dem Trägerquerschnitt mit der Querkraft \& um das Versatzmass

$$
v \cong 0.6(y+b)
$$

in Richtung des zunehmenden Momentes zu verschieben.

c) Die Längsschubarmierung kann im allgemeinen je zur Hälfte oben und unten in die Platte eingelegt und der Querbiegearmierung überlagert werden. Auf der Biegedruckseite der Platte kann die Längsschubarmierung entsprechend der gleichzeitig wirkenden Biegedruckkraft abgemindert werden.

d) Längs des ganzen Trägers ist in der Druckplatte je oben und unten eine konstruktive Mindestarmierung von 0.10 bis $0.15 \%$ einzulegen.

e) Die obere Schubspannungsgrenze ist auf rund 70 bis $80 \%$ des Wertes ohne Querbiegung abzumindern.

\section{Bemessung für den Gebrauchszustand (Rissesicherung)}

f) Der Vorspanngrad für die Beanspruchung "reine Querbiegung" und "Querbiegung mit Querzugkraft" wird mit

$$
k^{\prime}=\frac{M_{z U I, v}}{M_{g+p}}
$$

definiert. Bei gleichbleibender Vorspannung bewirkt die Querzugkraft $Z$, erfasst durch das Verhältnis $\gamma=Z \cdot d / M$, eine Abnahme des Vorspanngrades (Tabelle 7).

g) Der Vorspanngrad kann nach wirtschaftichen Gesichtspunkten festgelegt werden, wobei

- die Begrenzung nach unten im allgemeinen durch die Bedingung $k^{\prime} \geq M_{g} / M_{g}+p$ erfolgt (volle Vorspannung für ständige Lasten),

- die Begrenzung nach oben bedingt ist durch die Notwendigkeit, auch in vorgespannten Konstruktionen stets eine schlaffe Armierung anzuordnen.

h) Für ein gutes Risseverhalten ist die schlaffe Armierung in Form von eher vielen dünnen Stäben einzulegen. 
La référence [1] relate des essais de cinq poutres à Té en béton armé. Les dalles de compression étaient sollicitées pour deux poutres par un effort tranchant longitudinal isolé et pour les trois autres par un effort tranchant longitudinal accompagné d'une flexion transversale. Le présent rapport expose les résultats d'essais de deux nouvelies poutres, dont la dalle comprimée et partiellement précontrainte perpendiculairement à l'axe de la pièce. Ces deux récents essais permettent de mieux expliquer l'influence d'une précontrainte partielle appliquée simultanément aux sollicitations d'effort tranchant et de flexion transversale. Ce rapport s'attache particulièrement à la comparaison de poutres semblables, soit précontraintes, soit armées de manière conventionnelle.

Comme l'ont démontrés les essais décrits sous [1], le système triangulé peut être efficacement utilisé pour l'étude du comportement de la dalle de compression. Ce modèle sera d'ailleurs ici le seul appliqué et nous laisserons de côté la méthode de la thérie classique de la résistance des matériaux. L'armature transversale de la dalle (précontrainte et conventionnelle) fut déterminée à la base d'un dimensionnement plastique, de manière analogue aux poutres Q4 et $Q 5$ étudiées dans le rapport précédent [1].

Poutre Q6: Superposition des armatures issues du système triangulé et de la flexion transversale (fig. $5 a$ ).

Poutre Q7: Dimensionnement de l'armature transversale à la flexion accompagnée de la traction ( $f i g .5 b)$.

La précontrainte transversale de la dalle de compression fut maintenue constante tout au long de la poutre. Elle fut ainsi choisie, qu'avec une contrainte d'arête admissible de traction de $10 \mathrm{~kg} / \mathrm{cm}^{2}$, le degré de précontrainte $\mathrm{K}^{\prime}$ dans le domaine de la dalle sollicitée à la flexion transversale s'élève à 0.8 . Le degré de précontrainte atteindra respectivement la valeur 0.64 pour le domaine de la dalle sollicitée à la flexion transversale accompagnée de traction (Tab. 7).

Tous les autres paramètres, tel que qualité du béton, dimensions de la pièce, armatures de l'âme, etc. pouvant influencer les résultats furent maintenus constants.

L'avantage principal d'une précontrainte partielle perpendiculaire à l'axe de la pièce, par rapport à une solution composée uniquement d'armature d'acier conventionnelle, est sous aucun doute le meilleur comportement à la fissuration dans le domaine d'utilisation. Cette remarque est valable aussi bien pour une sollicitation combinée d'effort tranchant longitudinal et de flexion transversale, que pour une sollicitation d'effort tranchant isolé. Des fissures se forment d'ailleurs seulement pour de grandes charges. Leurs ouvertures ont été dans les présents essais avec dalles précontraintes pour des charges allant jusqu' à 1.4 fois la charge de service, plus petites que pour les mêmes dalles armées de manière conventionnelle. Dans le domaine de la charge théorique de rupture, la précontrainte transversale n'a plus d'influence sur l'armature transversale de la dalle. 
un comportement satisfaisant à la fissuration sous les charges de service. L'examen des résultats permet d'émettre les recommandations exposées ci-dessous.

\section{Dimensionnement à la rupture}

a) La capacité portante nécessaire de l'armature totale (précontrainte et conventionnelle) par unité de longueur de la poutre sera dimensionnée par les formules

$$
z=\frac{Q}{\cdot y} \cdot \frac{b-b_{0}}{b} \quad \text { resp. } z=\frac{Q}{\cdot y} \cdot \frac{b-b_{0}}{b}
$$

si l'on accepte dans quelques barres un écoulement avant que l'on atteigne la capacité portante à la flexion ou à l'effort tranchant de la poutre.

b) L'armature transversale sera par rapport à la section envisagée translatée dans la direction croissante des moments de flexion d'une quantité égale à

$$
v \cong 0.6(y+b)
$$

c) L'armature nécessaire à la reprise de l'effort tranchant sera généralement disposée également dans la nappe inférieure et supérieure de la dalle. L'armature nécessaire à la reprise de la flexion transversale y sera superposée. Une réduction de l'armature d'effort tranchant est permise dans la partie comprimée de la dalle d'une valeur correspondante à la force de compression de la flexion transversale.

d) L'armature minimale transversale de la dalle comprimée tout au long de la poutre sera au moins égale à $0.10 \div 0.15 \%$ pour chacune des deux nappes.

e) La contrainte tangentielle limite sera fixée à $75 \%$ de la valeur correspondante sous flexion transversale.

\section{Dimensionnement pour les charges de service (Fissuration)}

f) Le degré de précontrainte pour les sollicitations "flexion pure" et "flexion avec traction axiale" est définie par

$$
\kappa^{\prime}=\frac{M_{z U 1, V}}{M_{g+p}}
$$

La traction transversale peut être prise en compte par le paramètre $\gamma=Z \cdot d / M$ qui conduit à une diminution du degré de précontrainte (Tab. 7).

g) Le degré de précontrainte peut être fixé par des critères économiques, cependant:

- la limite inférieure est généralement déterminée par la condition $K^{\prime} \geq M_{g} / M_{g}{ } P$ (précontrainte totale pour les charges permanentes)

- la limite supérieure est donnée pas la nécessité de placer une armature conventionnelle constructive à côté de l'armature de précontrainte.

h) Un faible écartement des barres assure un meilleur comportement à la fissuration que peu de gros diamètres possédant un grand écartement relatif. 
Tests on five reinforced concrete T-section beams were reported in [1]. The compression flanges in two beams (Q1, Q2) were loaded for "longitudinal shear only". In the other three beams (Q3, Q4, Q5), the compression flanges were loaded for "longitudinal shear and transverse bending". In this report the results of tests on two further beams (Q6, Q7) are presented. The compression flanges of these beams were prestressed in the transverse direction. From these tests the influence of partial prestressing for longitudinal shear and transverse bending should become clearer (Fig. 12). Of particular interest is the comparison of similar beams with and without the transverse prestressing.

From the series of tests in [1] it was shown that design based upon the flange truss model was more suitable than that based upon the taking up of main tensile stresses. Therefore only the truss model was applied here in these later tests. The total required transverse reinforcement and prestressing steel in the flange was determined from a plastic design analogous to the earlier tested beams $Q 4$ and $Q 5$.

Beam Q6: "Superposition of reinforcement and prestressing steel from the flange truss model and from transverse bending" (Fig. 5a).

Beam Q7: "Reinforcement and prestressing steel for transverse bending with a transverse tensile force from the flange truss model" (Fig. 5b).

The transverse prestressing of the compression flange was executed unaltered along the length of the beam. It was chosen so that for an allowable edge tensile stress of $10 \mathrm{~kg} / \mathrm{cm}^{2}$, the calculated degree of prestressing $k$ ' had a value of 0.80 . This was for pure transverse bending of the flange. For transverse bending with a transverse tensile force from the longitudinal shear a value of $K^{\prime}=0.64$ was assumed (Tab. 7).

All other parameters such as beam dimensions, bending and shear reinforcement of the web, concrete quality etc. were taken unaltered from beams Q1 to Q5 (Fig. 6).

For "longitudinal shear with transverse bending" and also for "transverse bending only" the deciding advantage of partial transverse prestressing of the compression flange over the usage of only conventional reinforcement can be seen clearly. It lies in the significantly more favourable cracking behavior in the working load Iegion. Cracks first appeared at higher loads. Also until a load of 1.4 times the working load was reached, the cracks widths were smaller than those in the analogous flanges with only conventional reinforcement. In the region around calculated failure load, the transverse prestressing has no more influence upon the action of the transverse flange reinforcement.

The compression flanges should be designed for load capacity as well as for permissible crack behavior under working load conditions. From the test results the following preliminary recommendations can be made.

\section{Design for load capacity}

a) Following from longitudinal shear, the required yield capacity of prestressing 
steel and conventional reinforcement per unit length of beam can be designed from the formula

$$
z=\frac{Q}{5 \cdot y} \cdot \frac{b-b_{0}}{b} \text { or } \quad z=\frac{Q}{4 \cdot y} \cdot \frac{b-b_{o}}{b}
$$

depending on whether the yielding of a single reinforcement bar before the reaching of bending or web shear capacity is accepted or not.

b) The transverse reinforcement of the flange for a beam cross section with shear force $Q$ is displaced by a distance

$$
v \cong 0.6(y+b)
$$

in the direction of the increasing moment.

c) The longitudinal shear reinforcement in the flange can in general be placed with half as top reinforcement and half as bottom reinforcement. The transverse reinforcement can be superimposed upon this reinforcement. On the bending compression side of the flange the longitudinal shear reinforcement can be reduced by an amount corresponding to the simultaneous bending compression force.

d) A minimum reinforcement of $10-15 \%$ is placed top and bottom in the compression flange along the whole beam.

e) The maximum permissible shear strength is reduced to about $70-80 \%$ of the value for a beam without transverse bending.

\section{Design for working load conditions (crack control)}

f) The prestressing degree for the actions of "pure transverse bending" and "transverse bending with transverse tensile force" can be defined by

$$
k^{\prime}=\frac{M_{\text {allowable, }}}{M_{g+p}}
$$

With constant prestressing, the transverse tensile force $Z$, expressed by the relationship $\gamma=Z \cdot d / M$, causes a reduction of the prestressing degree

(Tab. 7).

g) The prestressing degree can be determined from an economical point of view, but

- the lower boundary in general results through the condition $k^{\prime} \geq M_{g} / M_{g+p}$ (full prestress for dead loads)

- the upper boundary is determined by the necessity in prestressed constructions to always place some conventional reinforcement.

h) For a favourable crack behavior, the reinforcement should preferrably consist of many small bars rather than fewer large bars. 
Der vorliegende Versuchsbericht ist im Rahmen der zweiten Phase des Forschungsprojektes "Teilweise Vorspannung im Brückenbau" ausgearbeitet worden. Dieses Programm wurde durch einen Beitrag aus dem

\section{Kredit zur Förderung der Strassenbauforschung}

des Eidgenössischen Departementes des Innern ermöglicht. Für diese grosszügige finanzielle Unterstützung möchten die Verfasser dem Departement des Innern und der Kommission für Strassenbauforschung mit ihrem Präsidenten, Herrn Dr. R. Ruckli, herzlich danken.

Den Firmen

Monteforno Stahl- und Walzwerke AG, Bodio

von Moos Stahl AG, Luzern

sind die Verfasser für die Zurverfügungsstellung von Armierungsstählen zu Dank verpflichtet.

Der Firma

Stahlton AG, Zürich

danken wir für ihre Unterstützung bei der Ausführung der Quervorspannung bestens.

Die Versuche konnten an der Eidg. Materialprüfungs- und Versuchsanstalt (EMPA) in Dübendorf durchgeführt werden. Hiefür sei Herrn Prof. Dr. Th. Erismann, Direktionspräsident, sowie Herrn Dr. M. Ladner, Vorsteher der Abteilung Massivbau, der beste Dank ausgesprochen.

Herr M. Baumann, dipl. Ing., betreute die messtechnischen Belange und Herr K. Bucher half bei der Durchführung der Versuche mit. Herr L. Sieger führte die Zeichnungen aus und Frl. S. Burki schrieb die Druckbogen. Für diese Unterstützung und die gute Zusammenarbeit danken die Verfasser herzlich. 
BEZEICHNUNGEN

\section{Geometrische Grössen}

\begin{tabular}{|c|c|}
\hline$H$ & Trägerhöhe \\
\hline h & statische Höhe \\
\hline$y, y_{p}$ & Hebelarme \\
\hline$b$ & Plattenbreite \\
\hline$b_{0}$ & Stegbreite \\
\hline d & Plattendicke \\
\hline$a$ & Schubspannweite \\
\hline$F_{\mathrm{e}}$ & schlaffe Plattenquerarmierung \\
\hline $\mathrm{F}_{\mathrm{s}}$ & vorgespannte Plattenquerarmierung \\
\hline$F_{e B}$ & Bügelarmierung \\
\hline$F_{\text {eL }}$ & Längsarmierung \\
\hline$t$ & Abstand \\
\hline$\emptyset$ & Durchmesser \\
\hline
\end{tabular}

\section{Kraftgrössen}

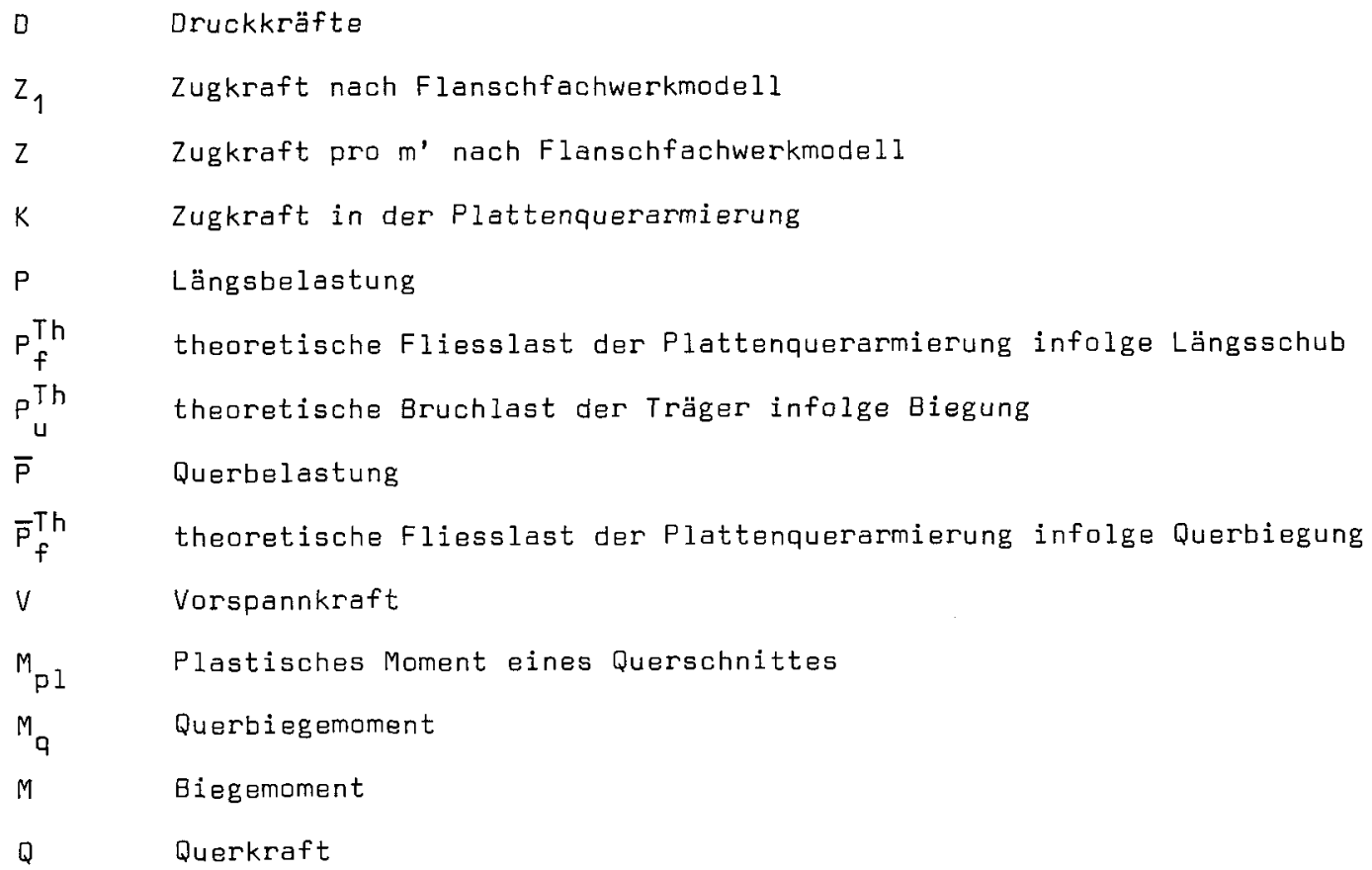

\section{Festigkeitswerte, Spannungen}

$\begin{array}{ll}\beta_{W} & \text { Würfeldruckfestigkeit } \\ \beta_{p} & \text { Prismendruckfestigkeit } \\ \beta_{b z} & \text { Biegezugfestigkeit } \\ \beta_{q z} & \text { Querzugfestigkeit } \\ \sigma_{e} & \text { Stahlspannung }\end{array}$




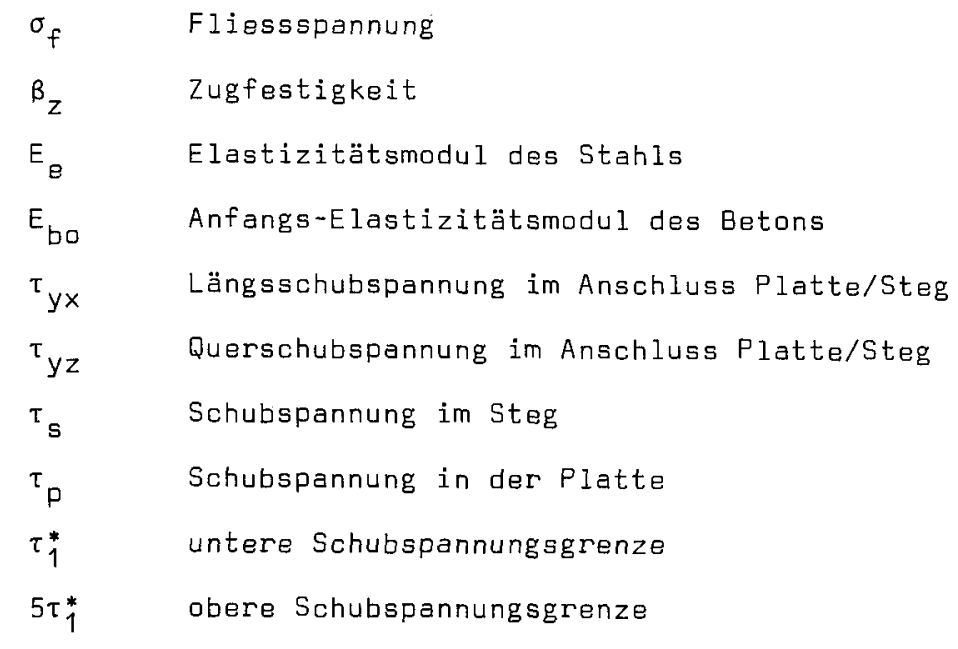

\section{Verformungen}

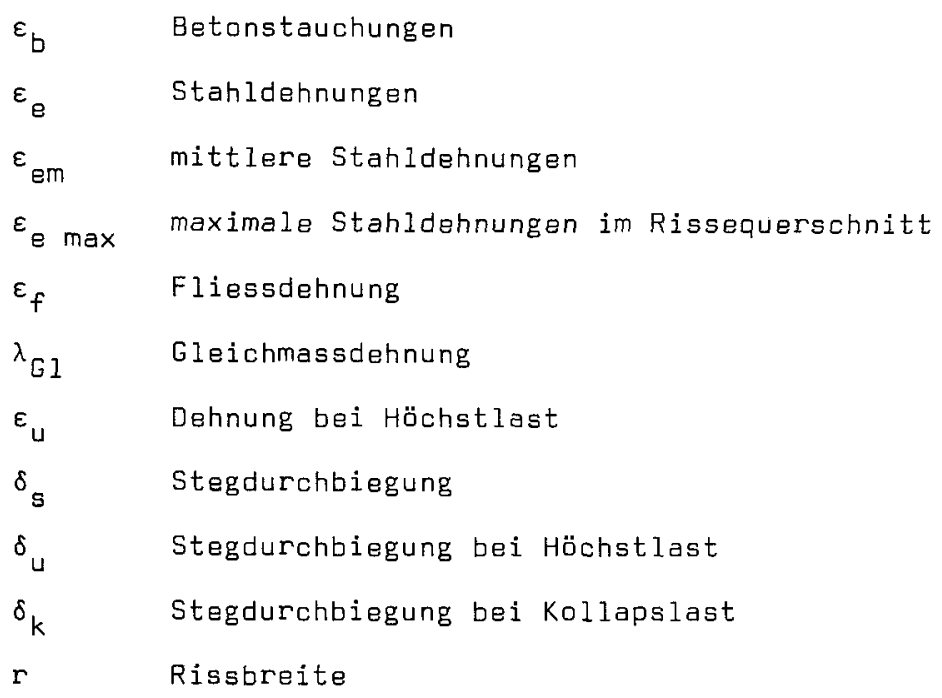

\section{Dimensionslose Parameter}

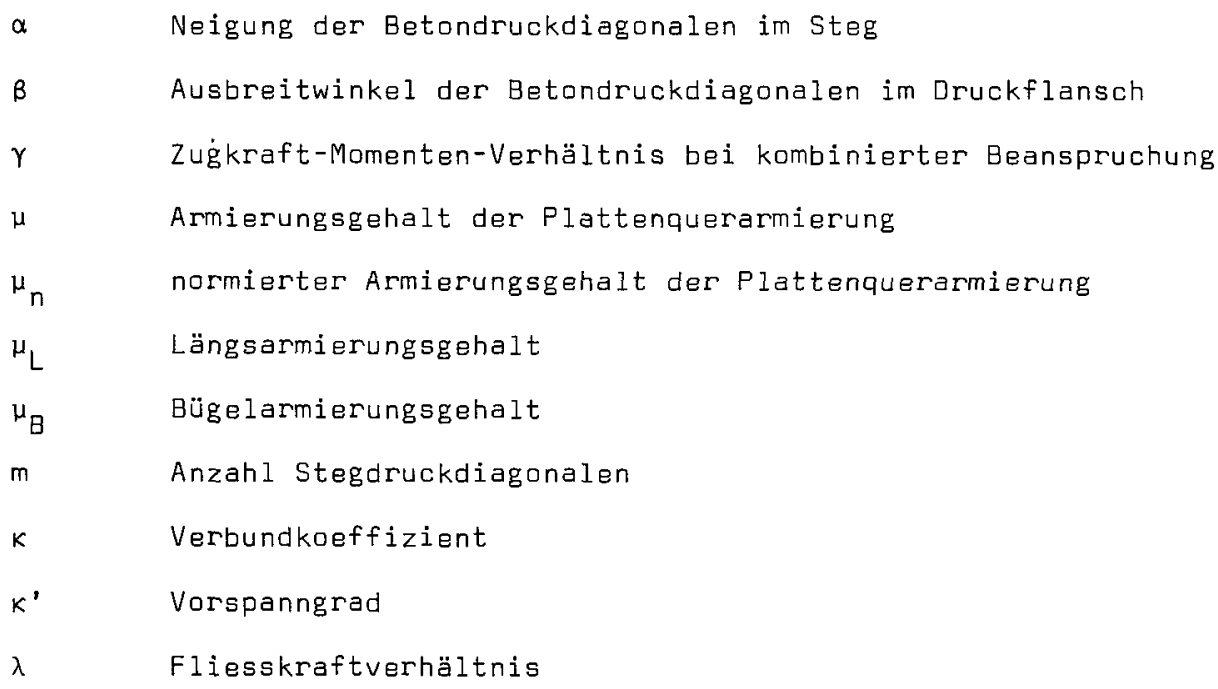




\section{Indizes}

\begin{tabular}{|c|c|}
\hline I, II, III & Stufen der Längs- bzw. Querbelastung \\
\hline b & Beton \\
\hline e & Stahl \\
\hline Th & theoretisch \\
\hline Ex & experimentell \\
\hline A & Anfangslast \\
\hline$E$ & Endlast \\
\hline$f$ & fliessen \\
\hline$u$ & Höchstlast \\
\hline k & Kollapslast \\
\hline d & dynamisch \\
\hline 5 & statisch, Spannstahl \\
\hline $\mathrm{g}$ & ständige Last \\
\hline$p$ & Nutzlast, in Platte \\
\hline ० & oben \\
\hline$u$ & unten \\
\hline L & längs \\
\hline$B$ & Bügel \\
\hline
\end{tabular}


[1] Badawy M., Bachmann H.: "Versuche über Längsschub und Querbiegung in Druckplatten von Betonträgern". Institut für Baustatik und Konstruktion, ETH Zürich, Bericht Nr. 6508-8, Juni 1977. Birkhäuser Verlag Basel und stuttgart.

[2] Badawy M.: "Längsschub und Querbiegung in Druckplatten von Stahlbeton-balken". Vortrag an der FBH/SIA-Tagung, ETH Zürich, Oktober 1976. Veröffentlicht in der Tagungsautographie, Verlag SIA Zürich. Bachmann H.: "Versuche über den Einfluss geneigter Spannglieder auf das Schubtragverhalten teilweise vorgespanter Betonbalken". Der Bauingenieur, 51. Jahrgang, Heft 7, Juli 1976. Institut für Baustatik und Konstruktion, ETH Zürich, Bericht Nr. 58, Birkhäuser Verlag Basel und Stuttgart, 1976.

Norm SIA 162 (1968): Norm für die Berechnung, Konstruktion und Ausführung von Bauwerken aus Beton, Stahlbeton und Spannbeton. Schweiz. Ingenieurund Architekten-Verein (SIA), Zürich. 
Klammerwerte = Voriationskoeffizienten in \%

Stähle

\begin{tabular}{|c|c|c|c|c|c|c|c|}
\hline $\begin{array}{l}\varnothing \\
\mathrm{mm}\end{array}$ & $\begin{array}{l}\mathrm{Fe} \\
\mathrm{cm}^{2}\end{array}$ & $\begin{array}{r}\sigma_{f d}\left(\varepsilon^{\prime}\right) \\
t / \mathrm{cm}^{2}\end{array}$ & $\begin{array}{c}\sigma_{f s}(\varepsilon=0) \\
t / c^{2}\end{array}$ & $\begin{array}{c}\beta_{z d}\left(\varepsilon^{\prime}\right) \\
t / \mathrm{cm}^{2}\end{array}$ & {$\left[\begin{array}{c}\beta_{z s}\left(\varepsilon^{\prime}=0\right) \\
t / \mathrm{cm}^{2}\end{array}\right.$} & $\begin{array}{l}\lambda_{g l} \\
\%\end{array}$ & Stahlqualität \\
\hline $\begin{array}{c}4 \\
\text { (12 Proben) }\end{array}$ & 0.1256 & $\begin{array}{c}17.90 \\
(1.1)\end{array}$ & $\begin{array}{r}17.20 \\
(1.2)\end{array}$ & $\begin{array}{r}19.70 \\
(1.5)\end{array}$ & $\begin{array}{c}18.80 \\
(1.7)\end{array}$ & $\begin{array}{c}3.7 \\
(0.9)\end{array}$ & SPANNDRAHT \\
\hline $\begin{array}{c}6 \\
\text { (12Proben) }\end{array}$ & $\begin{array}{r}0.281 \\
(20)\end{array}$ & $\begin{array}{l}4.92 \\
(5.4)\end{array}$ & $\begin{array}{l}4.75 \\
(5.7)\end{array}$ & $\begin{array}{l}5.42 \\
(6.4)\end{array}$ & $\begin{array}{l}5.18 \\
(7.0)\end{array}$ & $\begin{array}{c}6.6 \\
(2.1)\end{array}$ & TOR (wärmebehandelt) \\
\hline 12 & 1.08 & 5.19 & 4.95 & 7.00 & 6.66 & 16.2 & BOX - ULTRA \\
\hline 30 & 6.96 & 5.79 & 5.54 & 7.92 & & 13.8 & BOX - ULTRA \\
\hline
\end{tabular}

Tabelle 1: Eigenschaften der Stähle

\section{Beton}

\begin{tabular}{lll|}
\hline Sand $0-4 \mathrm{~mm}$ & $830 \mathrm{~kg} / \mathrm{m}^{3}$ \\
Sand $4-8 \mathrm{~mm}$ & $450 " ~$ \\
Kies $8-16 \mathrm{~mm}$ & $600 " ~$ \\
Zement & $300 ~ "$ \\
Wasser & $183 "$ \\
W/Z-Faktor & 0.61 \\
Raumgewicht & $2363 "$ \\
Konsistenz & plastisch \\
\hline
\end{tabular}

Tabelle 2: Zusammensetzung des Betons

\section{Zementmörtel}

\begin{tabular}{|c|c|c|}
\hline Sand $0-1 \mathrm{~mm}$ & \multicolumn{2}{|c|}{$740 \mathrm{~kg} / \mathrm{m}^{3}$} \\
\hline Sand $1-4 \mathrm{~mm}$ & 740 & $"$ \\
\hline Zement & 420 & $"$ \\
\hline Wosser & 252 & $"$ \\
\hline Zusatz (Verflüssiger & 2 & $"$ \\
\hline W/Z -Faktor & 0.60 & \\
\hline Raumgewicht & 2160 & $"$ \\
\hline Konsistenz & erdfe & \\
\hline
\end{tabular}

Tabelle 5: Zusammensetzung des Zementmörtels

\section{Beton}

\begin{tabular}{|c|c|c|c|c|}
\hline Träger & $\beta_{w}\left(\mathrm{~kg} / \mathrm{cm}^{2}\right)$ & $\beta_{\mathrm{p}}\left(\mathrm{kg} / \mathrm{cm}^{2}\right)$ & $\beta_{\mathrm{bz}}\left(\mathrm{kg} / \mathrm{cm}^{2}\right)$ & $\beta_{\mathrm{bz}}\left(\mathrm{kg} / \mathrm{cm}^{2}\right)$ \\
\hline$Q 6 / Q 7$ & 360 & 289 & 53 & 23 \\
\hline
\end{tabular}

Tabelle 3 : Konventionelle Festigkeitswerte

\begin{tabular}{|c|c|c|c|c|c|}
\hline$\beta_{\mathrm{pd}} / \beta_{\mathrm{w}}$ & $\beta_{\mathrm{ps}}^{2^{\prime} / \beta_{\mathrm{w}}}$ & $\beta_{\mathrm{ps}}^{30^{\prime}} / \beta_{\mathrm{w}}$ & $\varepsilon_{\mathrm{u}}$ & $\varepsilon_{\max }$ & $E_{\mathrm{bo}} / \sqrt{\beta_{\mathrm{w}}}$ \\
\hline 0.72 & 0.65 & 0.59 & $1.7 \% \circ$ & $3.0 \% \mathrm{0}$ & 18670 \\
\hline
\end{tabular}

Tabelle 4: Spannungs-Dehnungsverhalten der Betonprismen (Werte von Q 1-Q5)

Zementmörtel

\begin{tabular}{|c|c|c|}
\hline Träger & $\beta_{w}\left(\mathrm{~kg} / \mathrm{cm}^{2}\right)$ & $\beta_{\mathrm{b} z}\left(\mathrm{~kg} / \mathrm{cm}^{2}\right)$ \\
\hline$Q 6 / Q 7$ & 282 & 53 \\
\hline
\end{tabular}

Tabelle 6: Konventionelle Festigkeitswerte des Zementmörtels 
Vorspanngrad: $\kappa^{\prime}=\frac{M_{\text {zul.v }}}{M_{g+p}}$

$M_{\text {zul.v }}$ : zuld̈ssiges Moment für volle Vorspannung

$M_{g+p}$ : Moment unter Gebrauchslast $\left(\frac{M p l}{1.8}\right)$

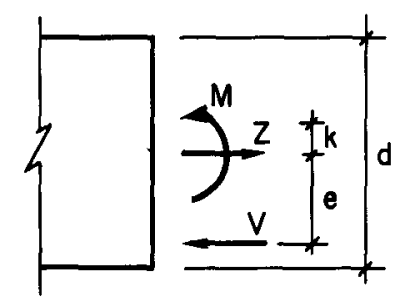

Querschnittswerte:

$d, e, F, k, W$

Querschnittskröfte :

$Z, M, V, y=\frac{Z}{M / d}$

Untere Betonrandspannung am ungerissenen Querschnitt:

$$
\begin{aligned}
& \sigma_{r}=\frac{M_{2 u l . v}}{W}+\frac{z}{F}-\frac{V \cdot e}{W}-\frac{V}{F} \\
& \sigma_{r}=M_{\text {zul.v }} \cdot \frac{1+\gamma \frac{k}{d}}{W}-v \cdot \frac{e+k}{W}
\end{aligned}
$$

Zulässiges Moment für volle Vorspannung :

$$
M_{z u l . v}=\frac{\sigma_{r} \cdot W+V(e+k)}{1+\gamma \frac{k}{d}}
$$

\begin{tabular}{|l|c|c|c|c|c|c|}
\hline \multirow{2}{*}{ Bereiche } & \multicolumn{5}{|c|}{ Betonsponnungen om Zugrand $\sigma_{\mathrm{r}}\left[\mathrm{kg} / \mathrm{cm}^{2}\right]$} \\
\cline { 5 - 7 } & $\gamma=\frac{Z}{\mathrm{M} / \mathrm{d}}$ & 0 & 10 & 20 & 30 & 40 \\
\hline \multirow{2}{*}{$\begin{array}{l}\text { reine Querbiegung } \\
\text { mit } \\
\text { Querzugkraft }\end{array}$} & 0 & 0.52 & 0.80 & 1.07 & 1.35 & 1.62 \\
\hline & 1 & 0.45 & 0.69 & 0.92 & 1.16 & 1.39 \\
\cline { 2 - 7 } & 1.5 & 0.42 & 0.64 & 0.86 & 1.08 & 1.30 \\
\hline
\end{tabular}

Tabelle 7 : Einfluss von zulässiger Betonzugspannung und Verhältnis $\frac{Z}{M / d}$ auf den Vorspanngrad $k$ 'der Druckplatten von $Q 6$ und $Q 7$ 


\begin{tabular}{|r|r|r|r|l|}
\hline \multicolumn{5}{|c|}{ Träger $Q 6$} \\
\hline 15 & $P_{A}(t)$ & $P_{E}(t)$ & $\bar{P}_{A}(t)$ & $\bar{P}_{E}(t)$ \\
\hline 1 & 1.0 & 1.0 & 0.16 & 0.16 \\
4 & 1.0 & 1.0 & 0.16 & 0.16 \\
5 & 1.0 & 1.0 & 1.64 & 1.64 \\
6 & 24.0 & 23.3 & 1.64 & 1.64 \\
7 & 34.0 & 23.0 & 2.28 & 2.15 \\
8 & 33.0 & 31.6 & 2.28 & 2.15 \\
9 & 43.0 & 32.3 & 2.91 & 2.79 \\
10 & 48.0 & 32.6 & 2.91 & 2.79 \\
11 & 51.0 & 41.5 & 2.91 & 2.75 \\
12 & 54.0 & 51.9 & 2.91 & 2.87 \\
13 & 56.0 & 53.3 & 2.91 & 2.78 \\
14 & 56.0 & 53.0 & 2.91 & 2.82 \\
15 & 56.0 & 52.4 & 2.91 & 2.84 \\
\hline
\end{tabular}

Tabelie 8 : Belastungsprotokoll

Träger Q 6

\begin{tabular}{|c|c|c|c|c|}
\hline \multicolumn{5}{|c|}{ Träger $Q 7$} \\
\hline LS & $P_{A}(t)$ & $P_{E}(t)$ & $\bar{P}_{A}(t)$ & $\bar{P}_{E}(t)$ \\
\hline 1 & 1.0 & 1.0 & 0.16 & 0.16 \\
3 & 1.0 & 1.0 & 0.16 & 0.16 \\
4 & 1.0 & 1.0 & 1.64 & 1.64 \\
5 & 24.0 & 233 & 1.64 & 1.64 \\
6 & 34.0 & 23.7 & 2.28 & 2.15 \\
7 & 33.0 & 31.7 & 2.28 & 2.15 \\
8 & 33.0 & 31.8 & 2.91 & 2.78 \\
9 & 43.0 & 41.4 & 2.91 & 2.75 \\
10 & 48.0 & 46.2 & 2.91 & 2.78 \\
11 & 51.0 & 49.7 & 2.91 & 2.74 \\
12 & 54.0 & 52.3 & 2.91 & 2.78 \\
13 & 56.0 & 53.7 & 2.91 & 2.81 \\
14 & 56.0 & 53.2 & 2.91 & 2.82 \\
15 & 56.0 & 53.0 & 2.91 & 2.83 \\
\hline
\end{tabular}

Tabelle 9 : Belastungsprotokoll Träger Q7

\begin{tabular}{|c|c|c|c|c|c|c|c|c|}
\hline \multirow{5}{*}{ 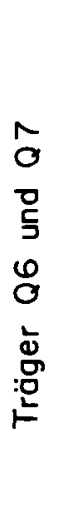 } & \multicolumn{6}{|c|}{ Theoretische Werte } & \multirow{2}{*}{\multicolumn{2}{|c|}{$\frac{\text { Exp. Werte }}{\text { Gemessen }}$}} \\
\hline & \multicolumn{4}{|c|}{ Vorberechnet } & \multicolumn{2}{|c|}{ Nachberechnet } & & \\
\hline & \multicolumn{2}{|c|}{ Steg } & \multicolumn{2}{|c|}{ Platte } & Steg & Platte & Steg & Platte \\
\hline & $\begin{array}{c}\text { Bruchlast } \\
(t)\end{array}$ & $\begin{array}{c}\tau_{s} \\
\left(\mathrm{~kg} / \mathrm{cm}^{2}\right)\end{array}$ & $\begin{array}{l}\text { Fliesslas } t \\
\qquad(t)\end{array}$ & $\begin{array}{c}\tau_{p} \\
\left(\mathrm{~kg} / \mathrm{cm}^{2}\right)\end{array}$ & $\begin{array}{c}\text { Bruchlast } \\
(t)\end{array}$ & $\begin{array}{l}\text { Fliessiast } \\
\qquad(t)\end{array}$ & $\begin{array}{c}\text { Bruchlast } \\
(\dagger)\end{array}$ & $\begin{array}{c}\text { Fliesslost } \\
(t)\end{array}$ \\
\hline & $P_{u}^{T h}=55$ & 50 & $\begin{array}{l}P_{f}^{T h}=43 \\
\vec{P}_{f}^{T h}=2.91\end{array}$ & $\begin{array}{l}\tau_{y x}=34.2 \\
\tau_{y z}=3.8\end{array}$ & $P_{u A}^{T h}=60$ & $\begin{array}{l}P_{f A}^{T h}=44.2 \\
\bar{P}_{f A}^{T h}=3.0\end{array}$ & $P_{U A}^{E x}=56$ & $P_{f A}^{E X} \simeq 53$ \\
\hline
\end{tabular}

Tabelle 10: Rechnerische und experimentelle Werte 


\begin{tabular}{|c|c|c|c|c|c|c|c|}
\hline & & \multicolumn{3}{|c|}{$\left(K_{\text {Tot }}^{E x}-K_{M_{q}}^{T h}\right):\left(K_{\text {Tot }}^{T h}-K_{M_{q}}^{T h}\right)$} & \multicolumn{3}{|c|}{$\left(K_{\text {Tot,neu }}^{\text {Ex }}-K_{M_{q}}^{T h}\right):\left(K_{\text {Tot }}^{T h}-K_{M_{q}}^{T h}\right)$} \\
\hline & & Theorie & $\begin{array}{l}\text { Geme } \\
\text { Spitzenwerte }\end{array}$ & $\begin{array}{l}\text { sene } \\
\text { Miftelwerte }\end{array}$ & Theorie & $\begin{array}{r}\text { Gemes } \\
\text { Spitzenwerte }\end{array}$ & $\begin{array}{l}\text { sene } \\
\text { Mittelwerte }\end{array}$ \\
\hline \multirow{2}{*}{$\begin{array}{c}\text { Träger } \\
\text { Q6 }\end{array}$} & linker Teil & $100 \%$ & $68 \%$ & $52 \%$ & $100 \%$ & $98 \%$ & $70 \%$ \\
\hline & rechter Teil & $100 \%$ & $86 \%$ & $62 \%$ & $100 \%$ & $100 \%$ & $81 \%$ \\
\hline \multirow{2}{*}{$\begin{array}{c}\text { Träger } \\
\text { Q7 }\end{array}$} & linker Teil & $100 \%$ & $82 \%$ & $68 \%$ & $100 \%$ & $100 \%$ & $82 \%$ \\
\hline & rechter Teil & $100 \%$ & $62 \%$ & $55 \%$ & $100 \%$ & $83 \%$ & $68 \%$ \\
\hline & & \multicolumn{3}{|c|}{$Z=\frac{Q}{4 y}$} & \multicolumn{3}{|c|}{$z=\frac{Q}{4 y} \cdot \frac{b-b_{0}}{b}$} \\
\hline
\end{tabular}

Tabelle 11: Gemessene Zugkräfte der oberen Plattenarmierung verglichen mit den theoretischen Fliesskräften nach Flanschfachwerkmodellen im Bereich "kombinierte Beanspruchung" 

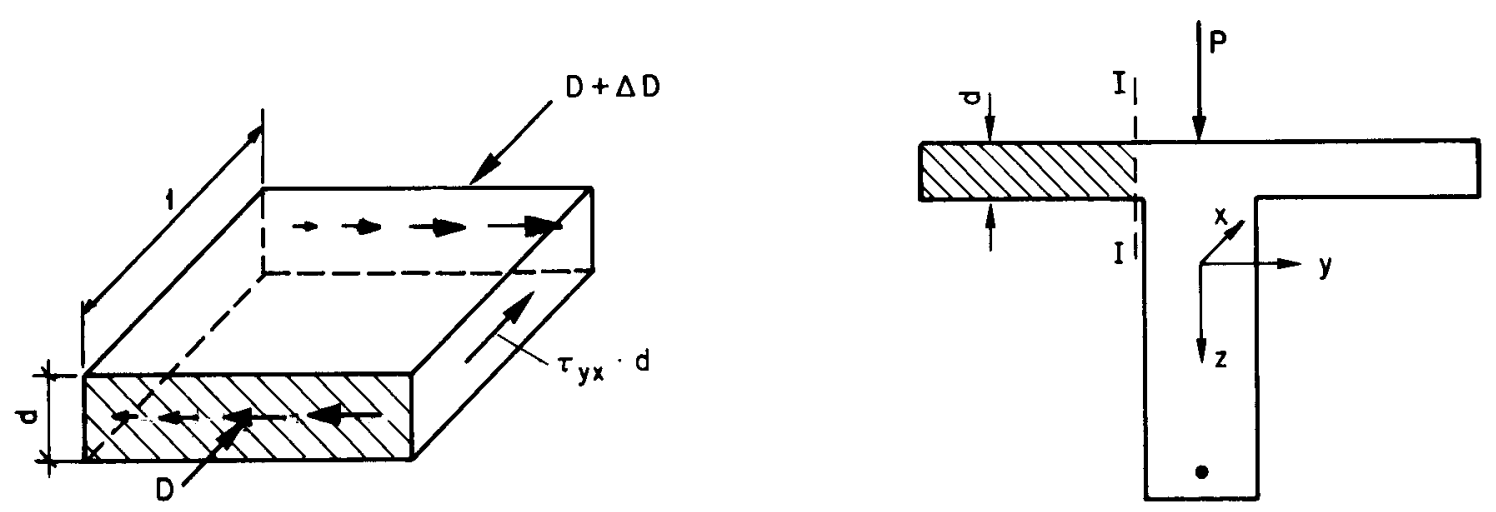

$\underline{\text { a-Löngsschub allein }}$
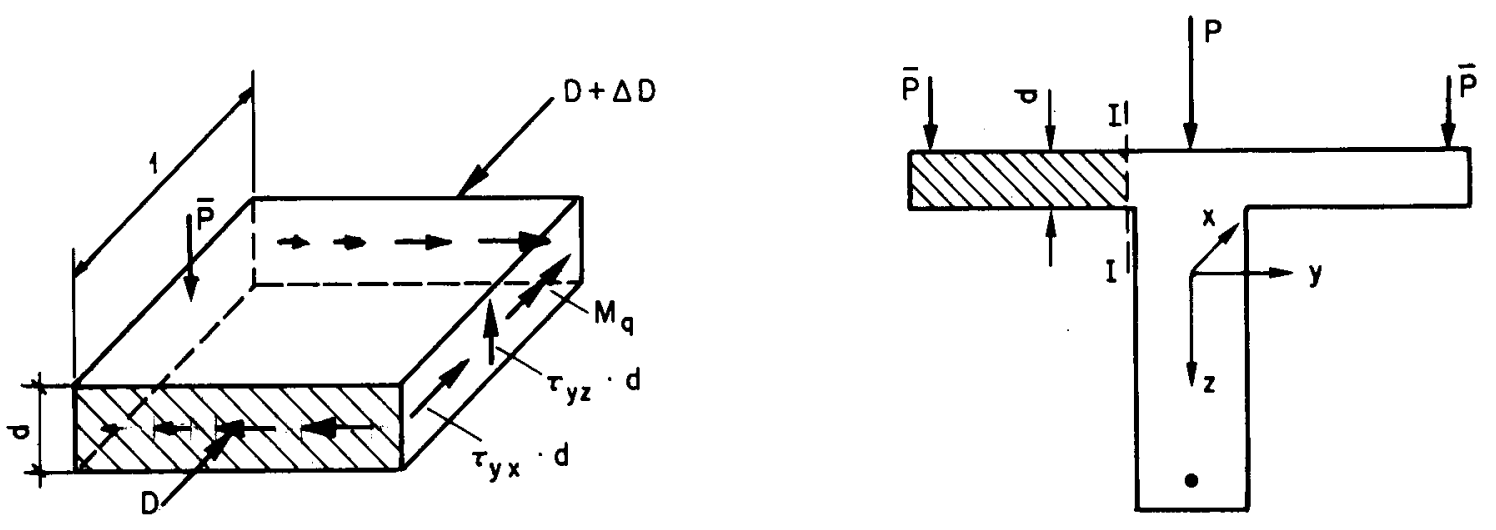

b-Löngsschub + Querbiegung
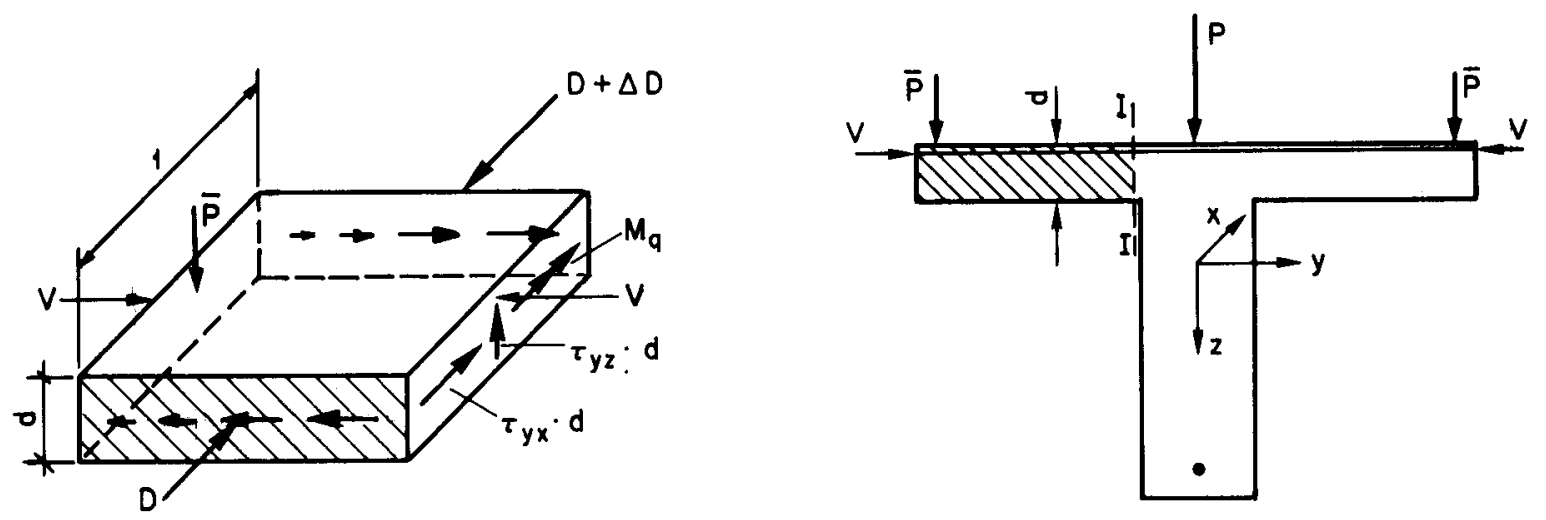

c-Löngsschub + Querbiegung + Quervorspannung

Bild 1: Problemstellung 
Räumliches Fachwerk

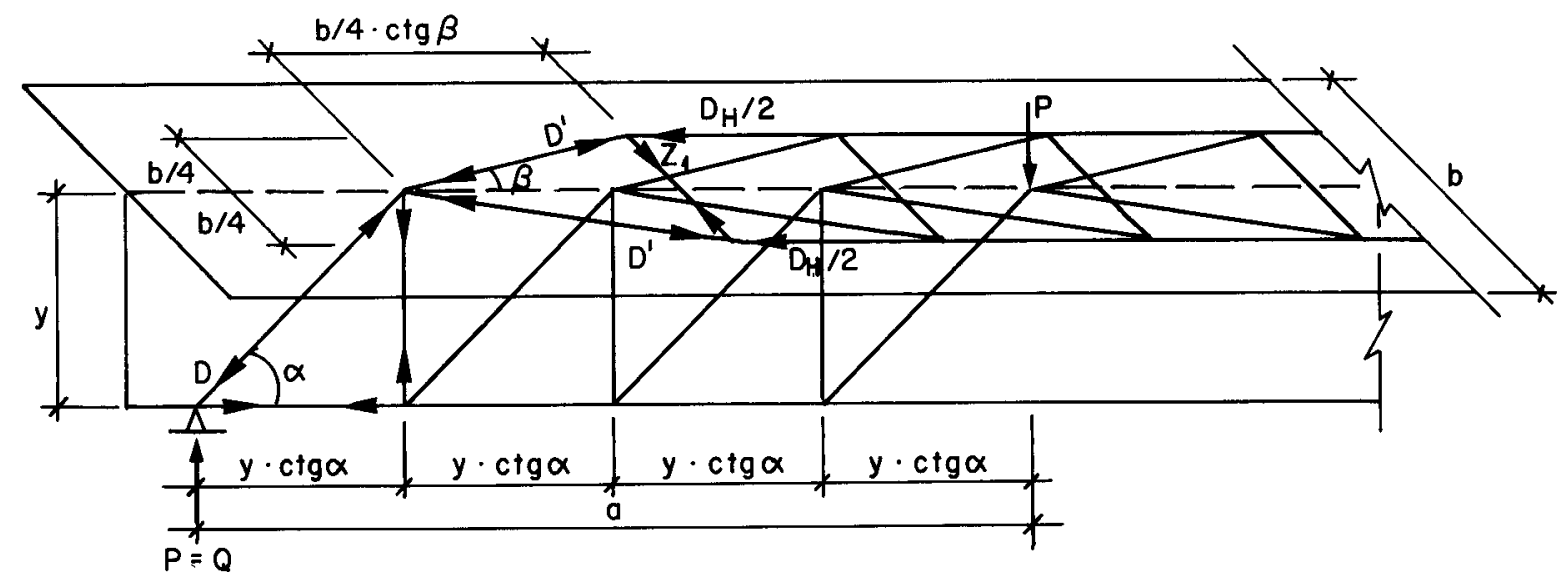

$D=\frac{Q}{\sin \alpha} ; \frac{D_{H}}{2}=\frac{D}{2} \cos \alpha=\frac{Q}{2} \operatorname{ctg} \alpha$

$Z_{1}=\frac{D_{H}}{2} \cdot \operatorname{tg} \beta=\frac{Q}{2} \operatorname{ctg} \alpha \cdot \operatorname{tg} \beta$

$Z_{\text {prom' }}=\frac{Q}{2 \cdot y} \operatorname{tg} \beta$

Kraftausbreitung in Scheiben

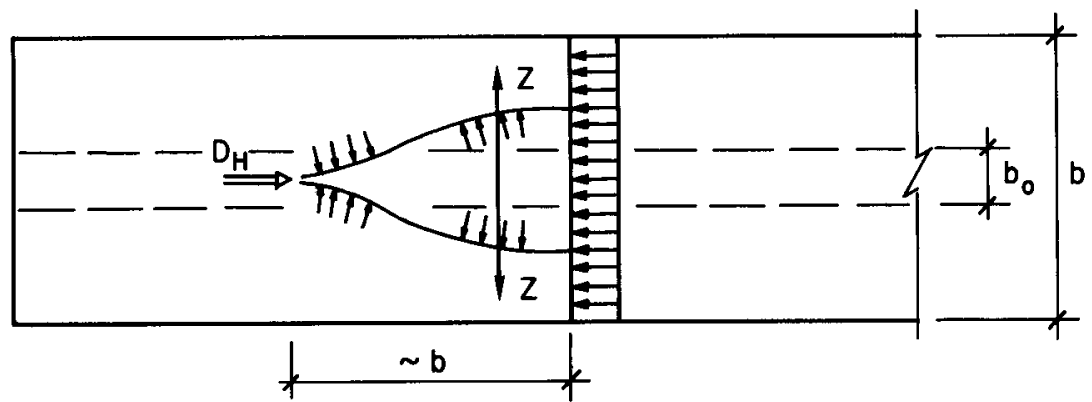

$Z \simeq \frac{D_{H}}{4} ; \operatorname{tg} \beta \simeq 0,5$

$\rightarrow Z_{\text {prom }}=\frac{Q}{4 \cdot y}$

Einfaches Flanschfachwerk

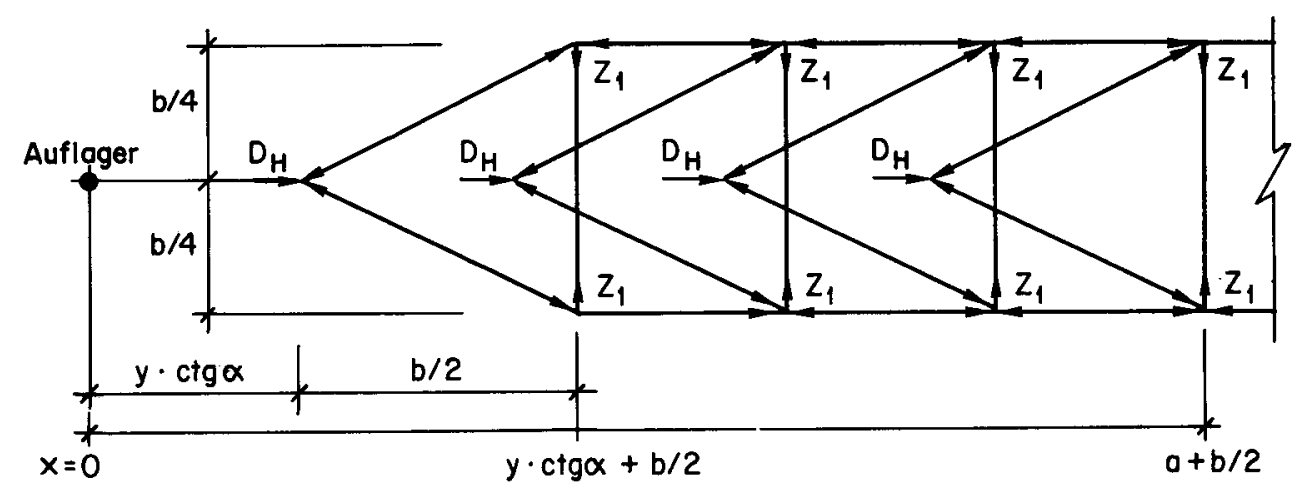

Bild 2 : Flanschfachwerk - Modell 


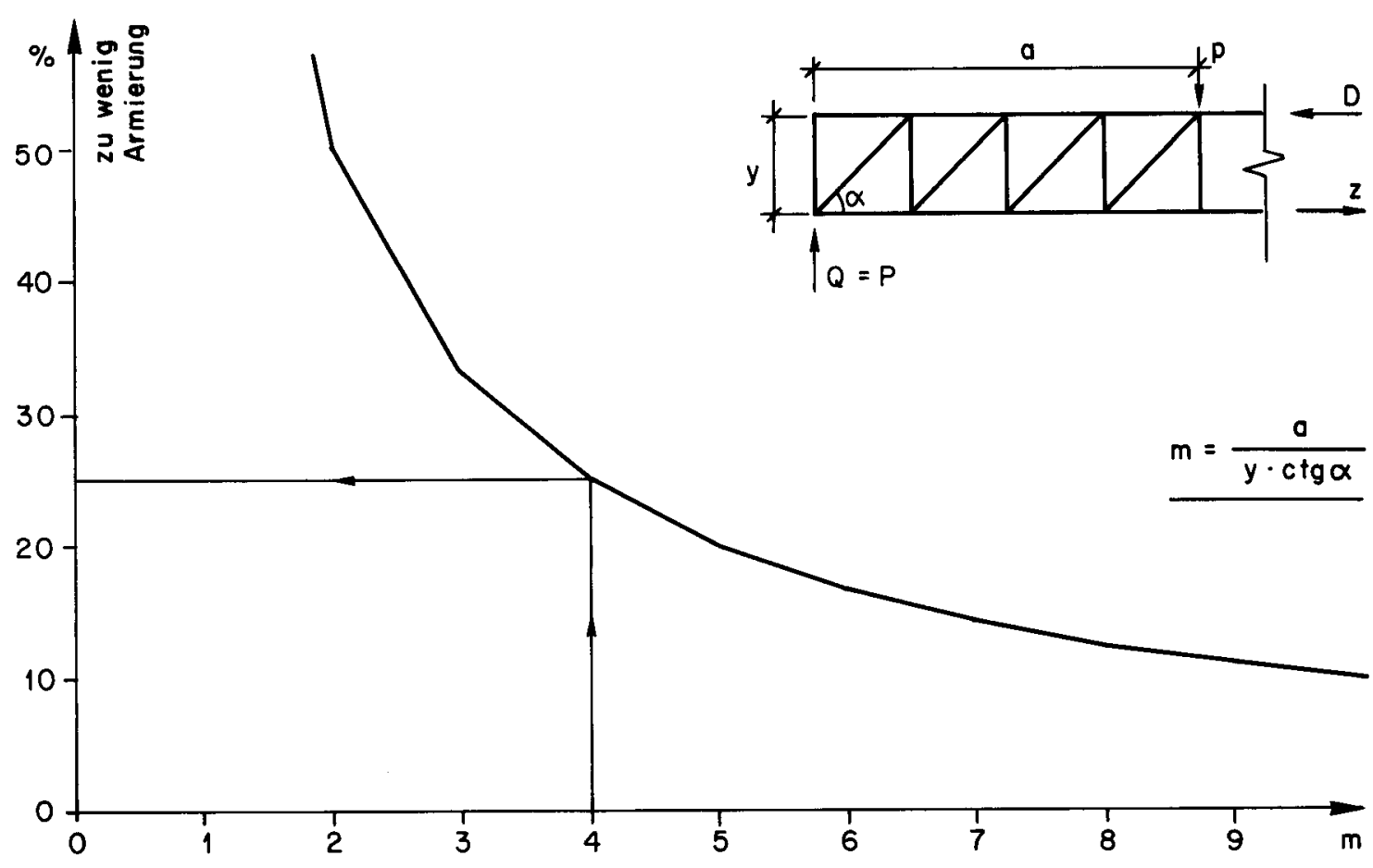

Bild 3: Einfluss des Verhältnisses $\frac{a}{y \cdot \operatorname{ctg} \alpha}$ auf die Gesamtarmierung

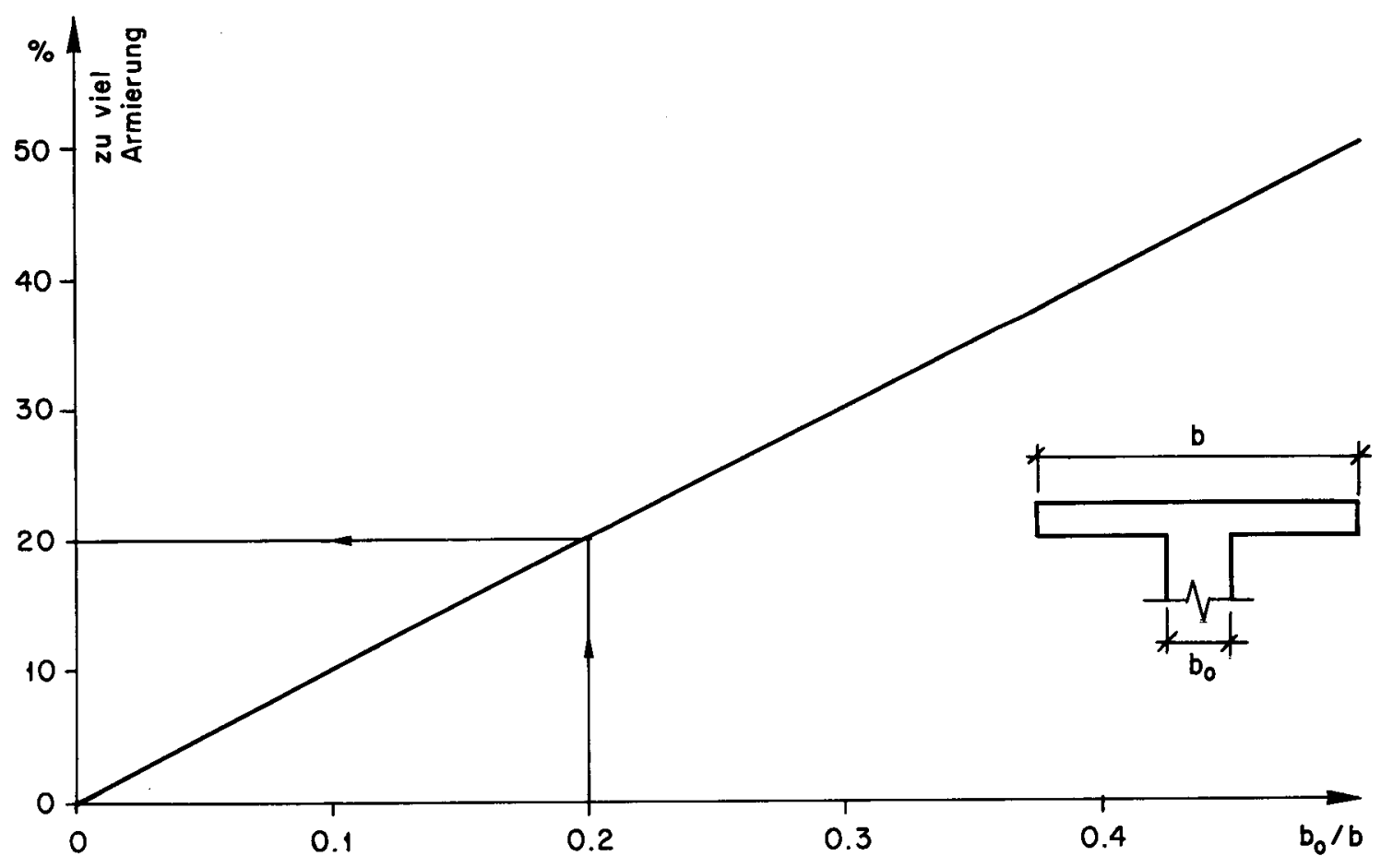

Bild 4: Einfluss des Verhältnisses $\frac{b_{o}}{b}$ auf die Armierung 
a) Superposition der Armierungen aus "Flanschfachwerk" und Querbiegung
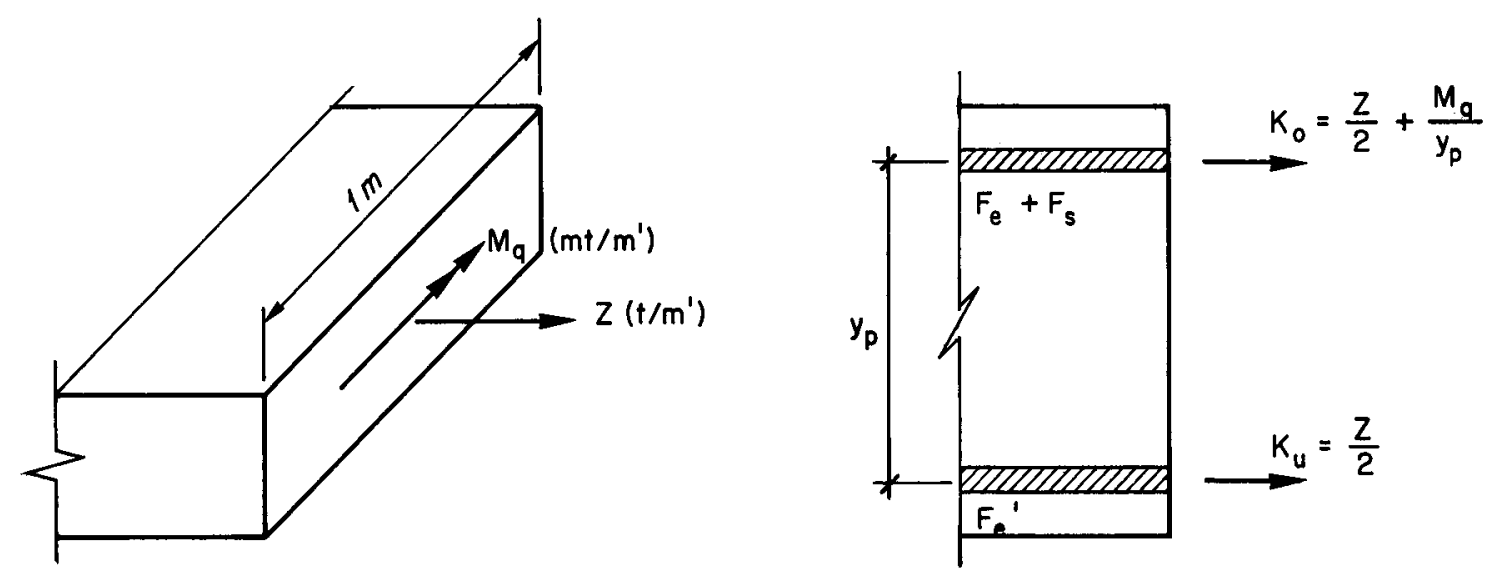

$y_{p}$ : innerer Hebelarm

b) Armierung für Querbiegung mit Querzugkraft aus "Flanschfachwerk"
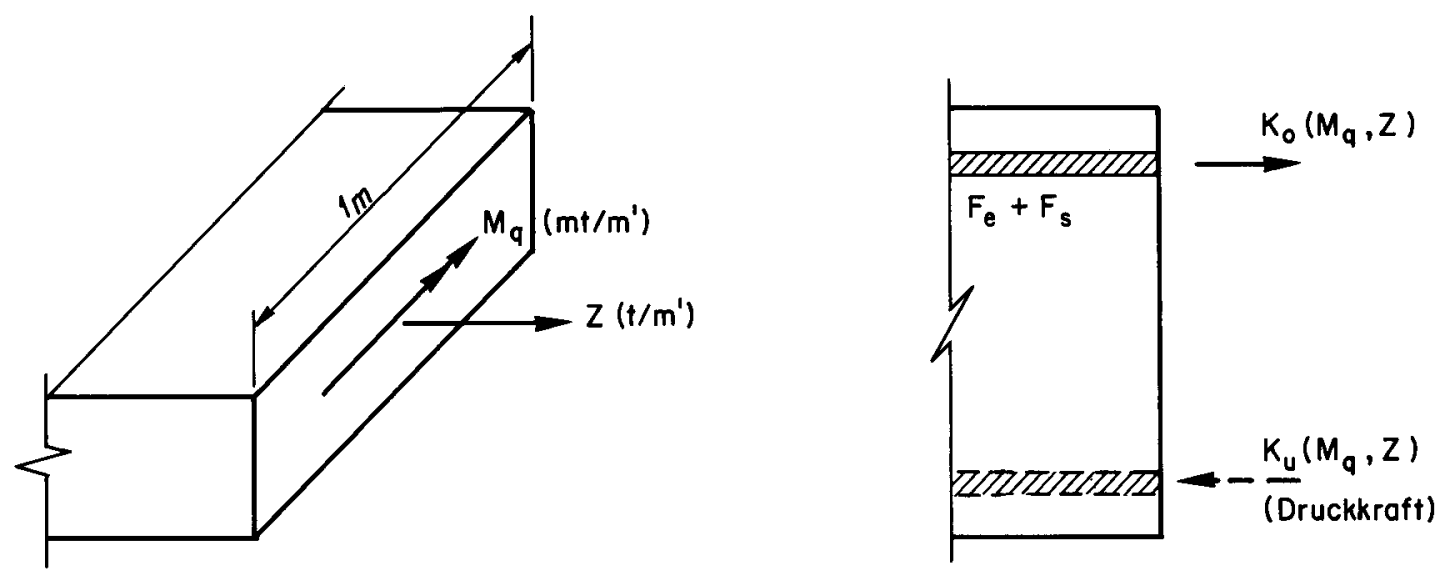

Bild 5: Bemessungsmodelle für Längsschub mit Querbiegung 

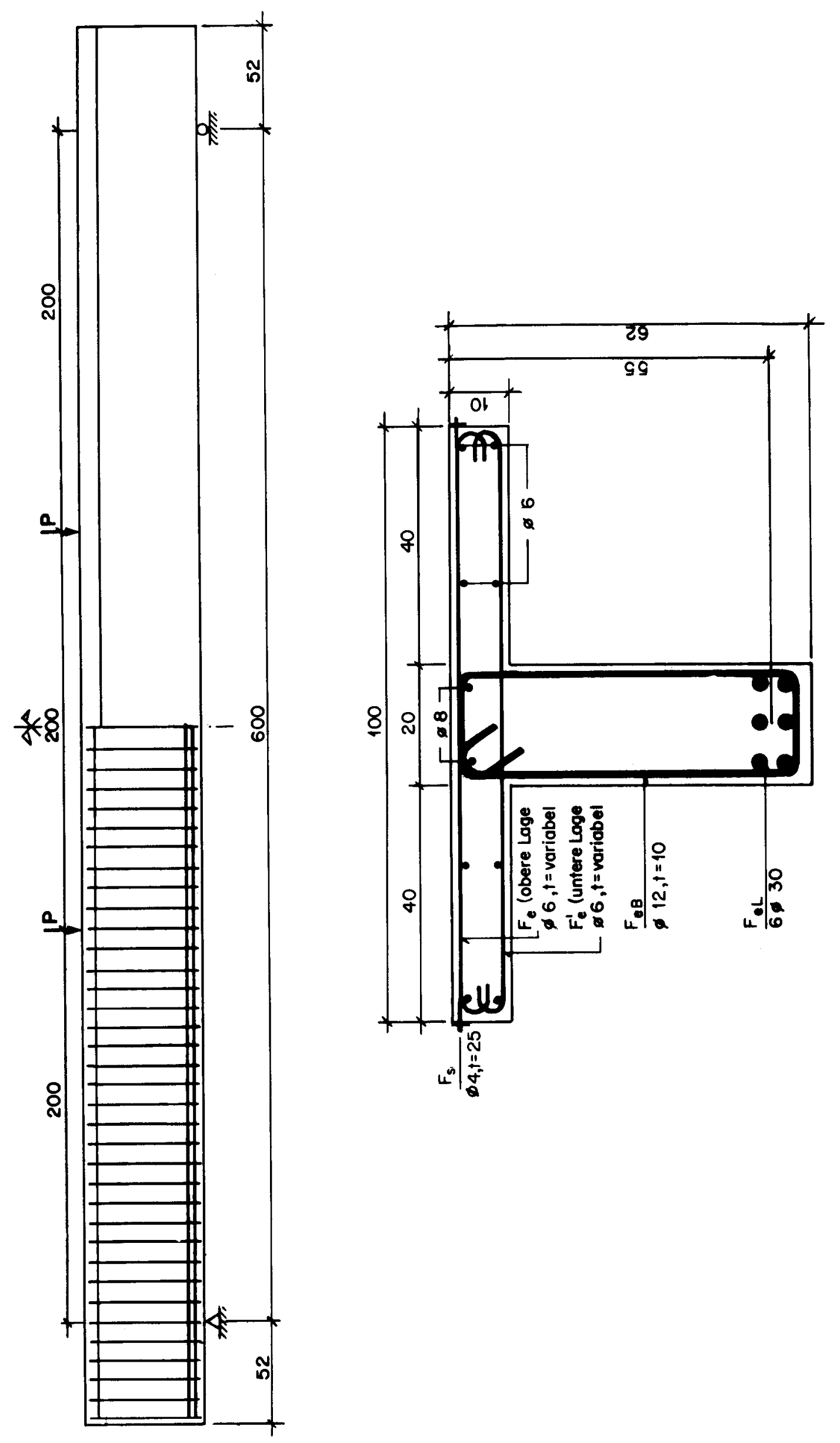

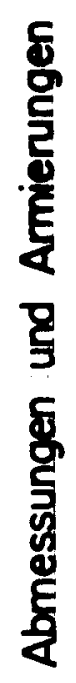
$\ddot{\varphi}$ 믐 

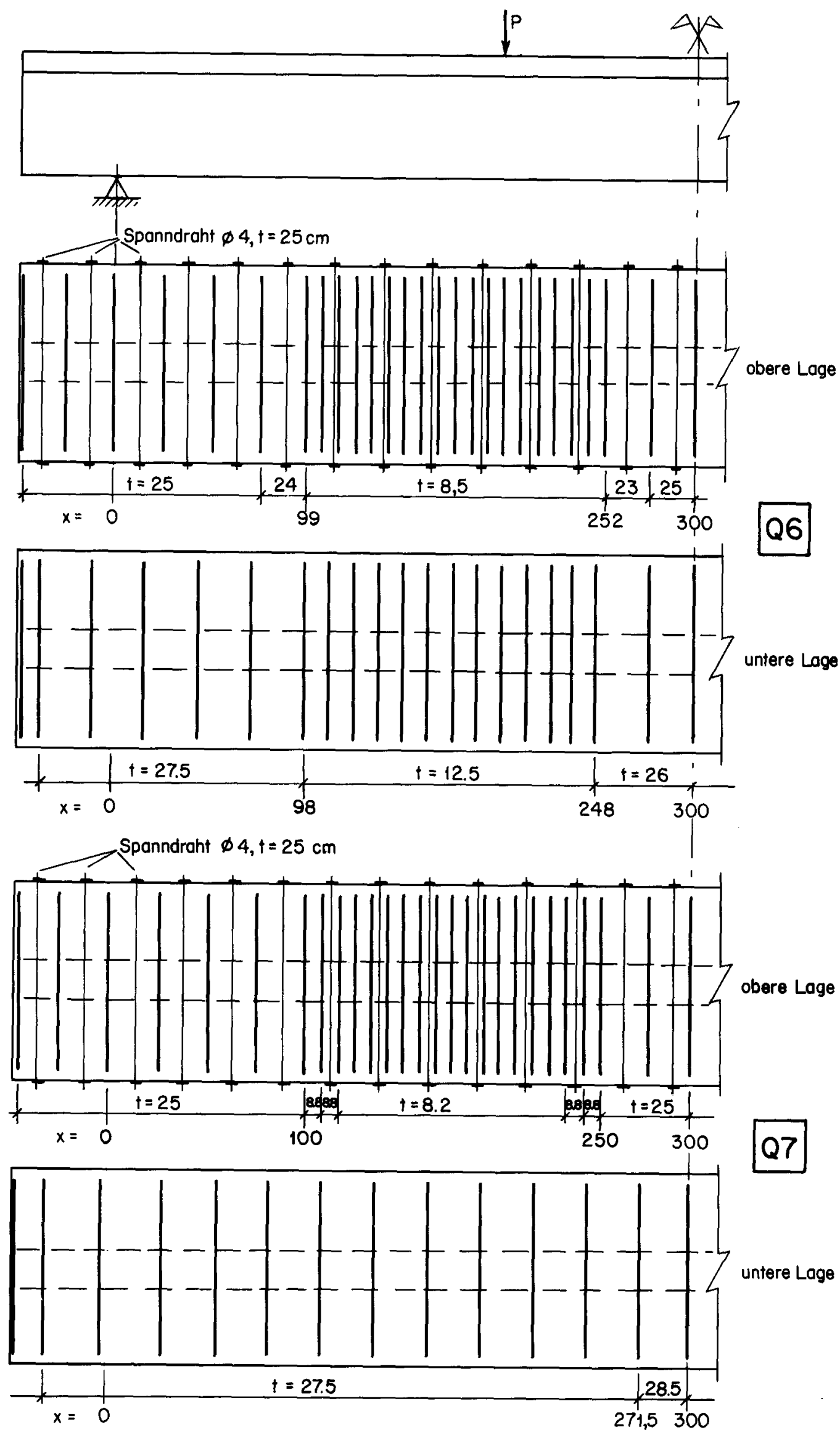

Bild 7: Plattenarmierung $\left(F_{e}, F_{e}^{\prime}=\varnothing 6, F_{s} \varnothing 4\right)$ 

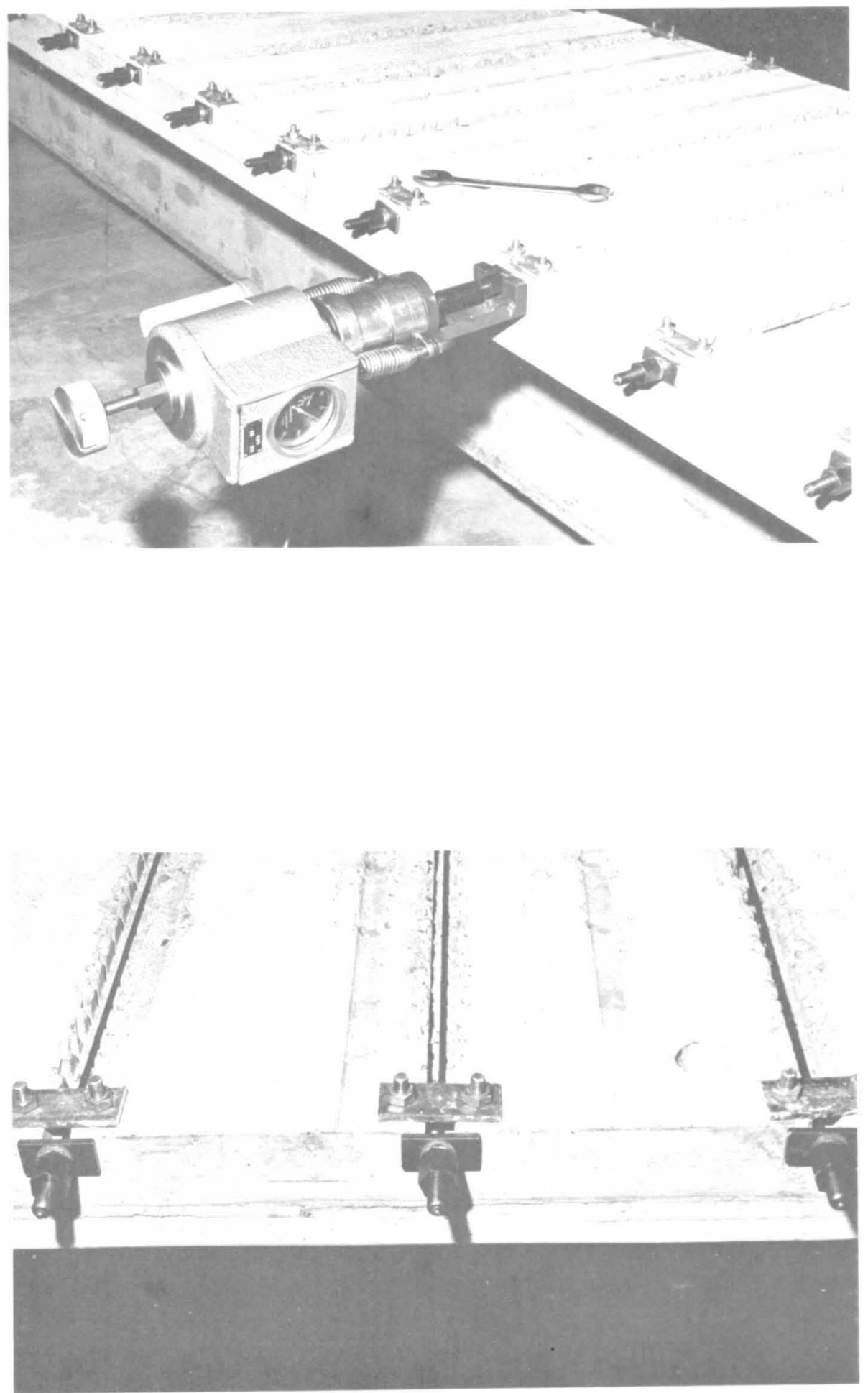

Bild 8: Quervorspannung der Platte 


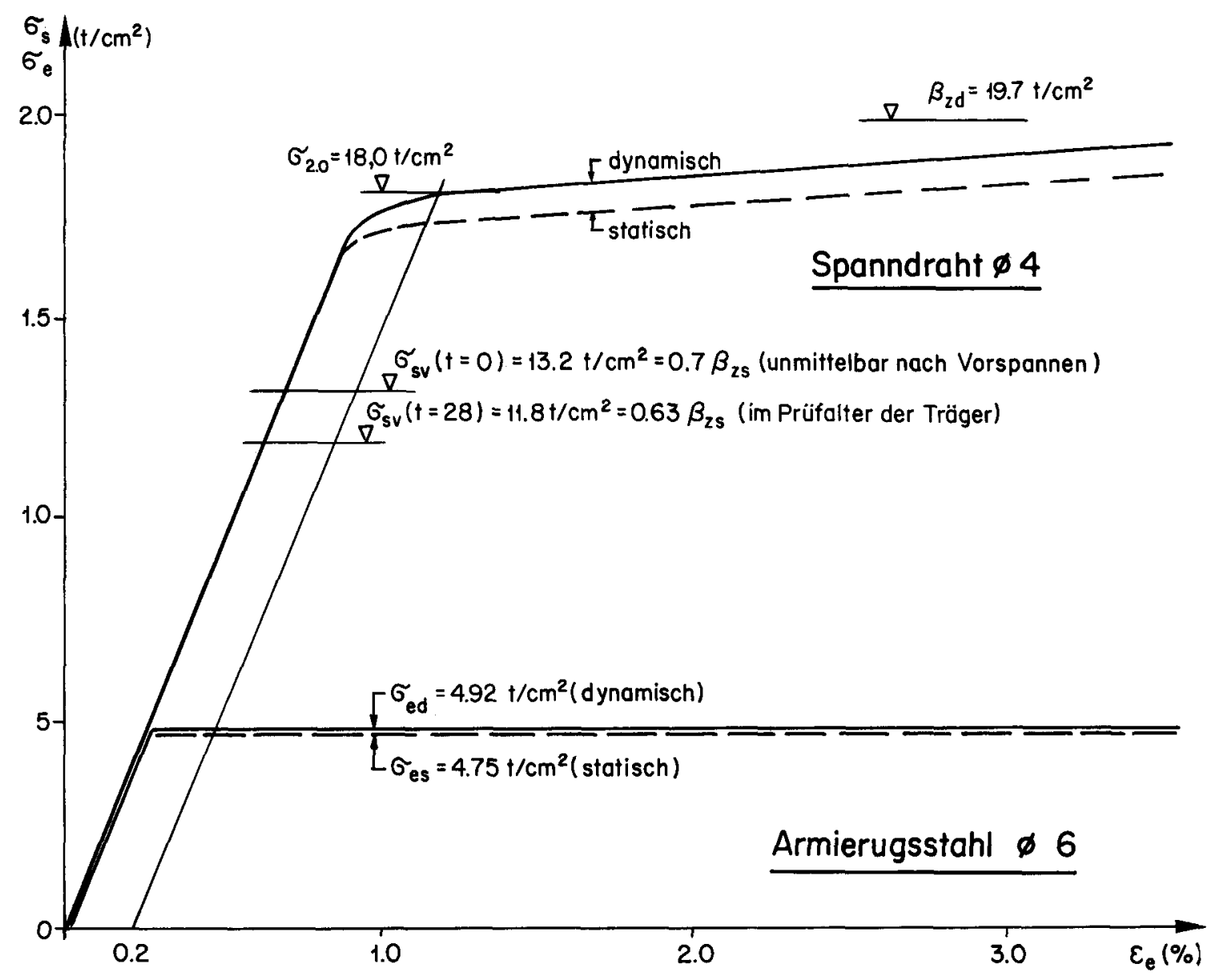

Bild 9: $\widetilde{\sigma}-\varepsilon$-Diagramme der Plattenarmierung

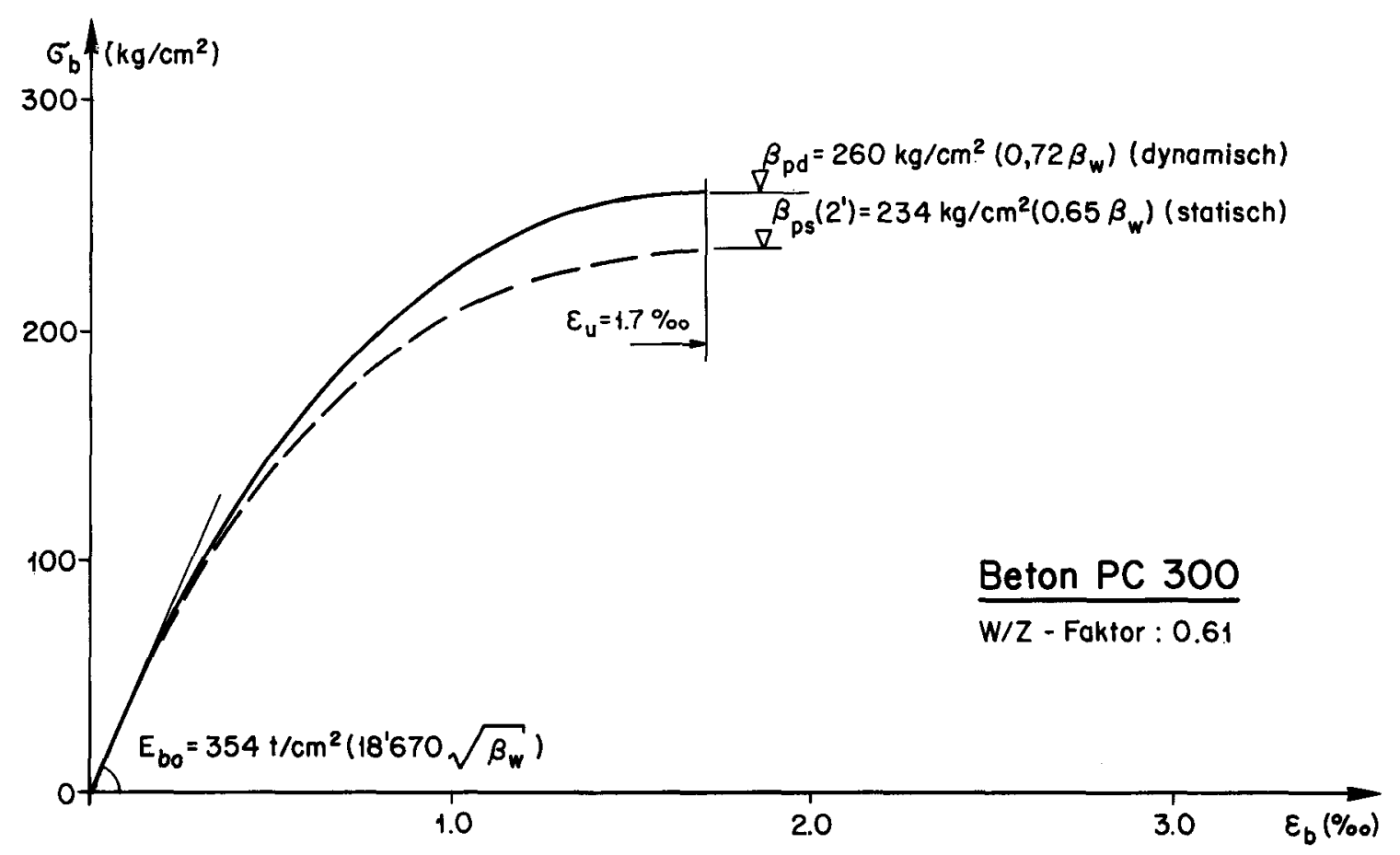

Bild 10: $\sigma-\varepsilon$-Diagramm des Betons 


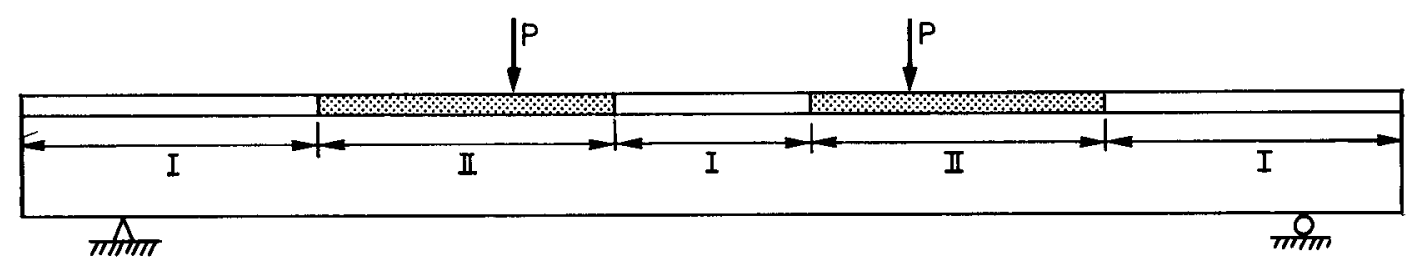

I Bereiche "reine Querbiegung"

II Bereiche „kombinierte Beanspruchung"

Balken Q6 $\quad F_{e}=F_{e}\left(M_{q}\right)+F_{e}(Z)$

WIA theoretisch erforderliche Fliesskroft

$\square$ konstruktive Minimalarmierung

Fliesskraft der oberen Armierungslage

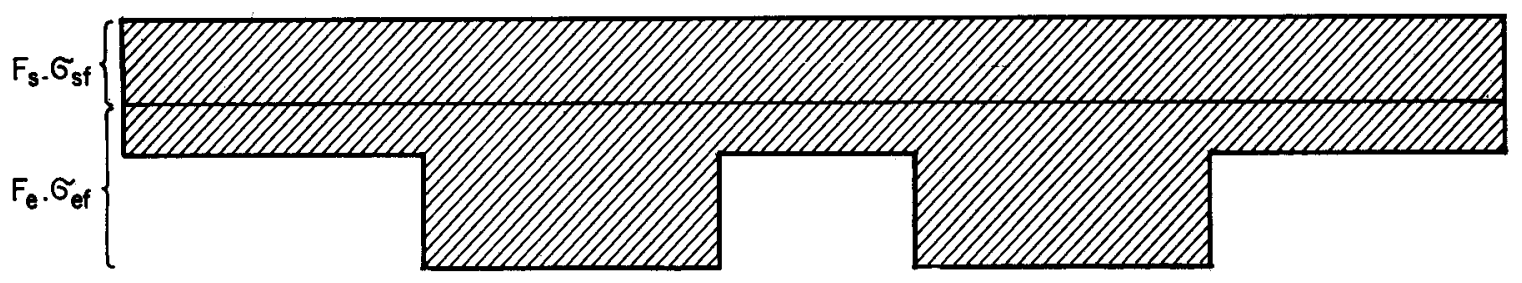

Fliesskraft der unteren Armierungslage

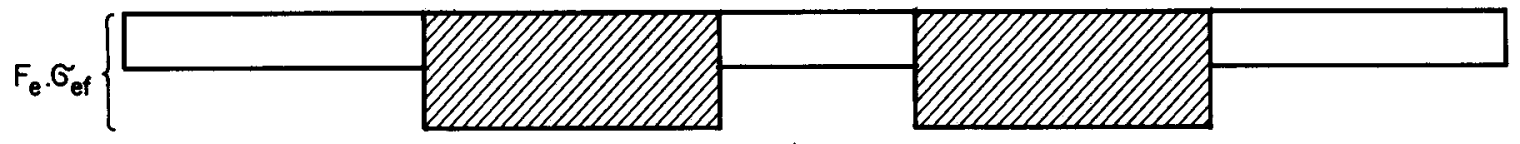

Balken Q7 $F_{e}=F_{e}\left(M_{q}+Z\right)$

Fliesskraft der oberen Armierungslage

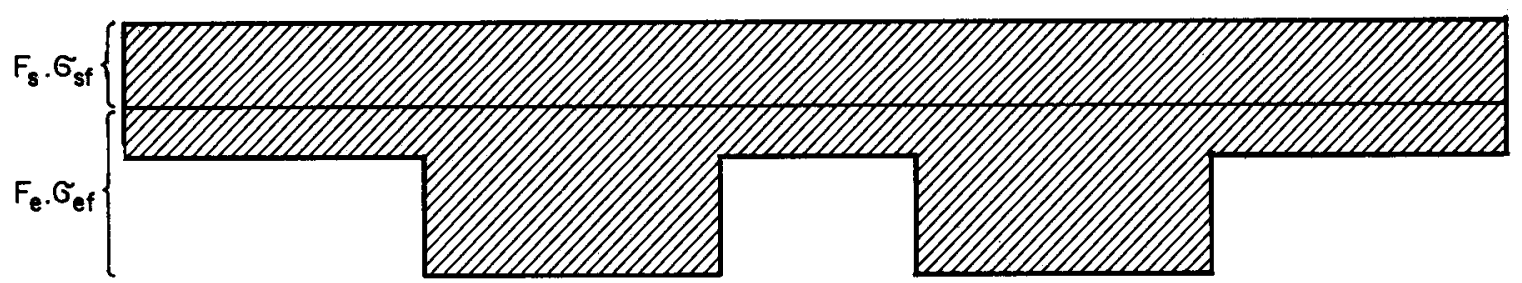

Fliesskraft der unteren Armierungslage

$F_{\mathrm{e}} \cdot \sigma_{\mathrm{ef}}\{$

Bild 11: Fliesskräfte der Plattenarmierung 

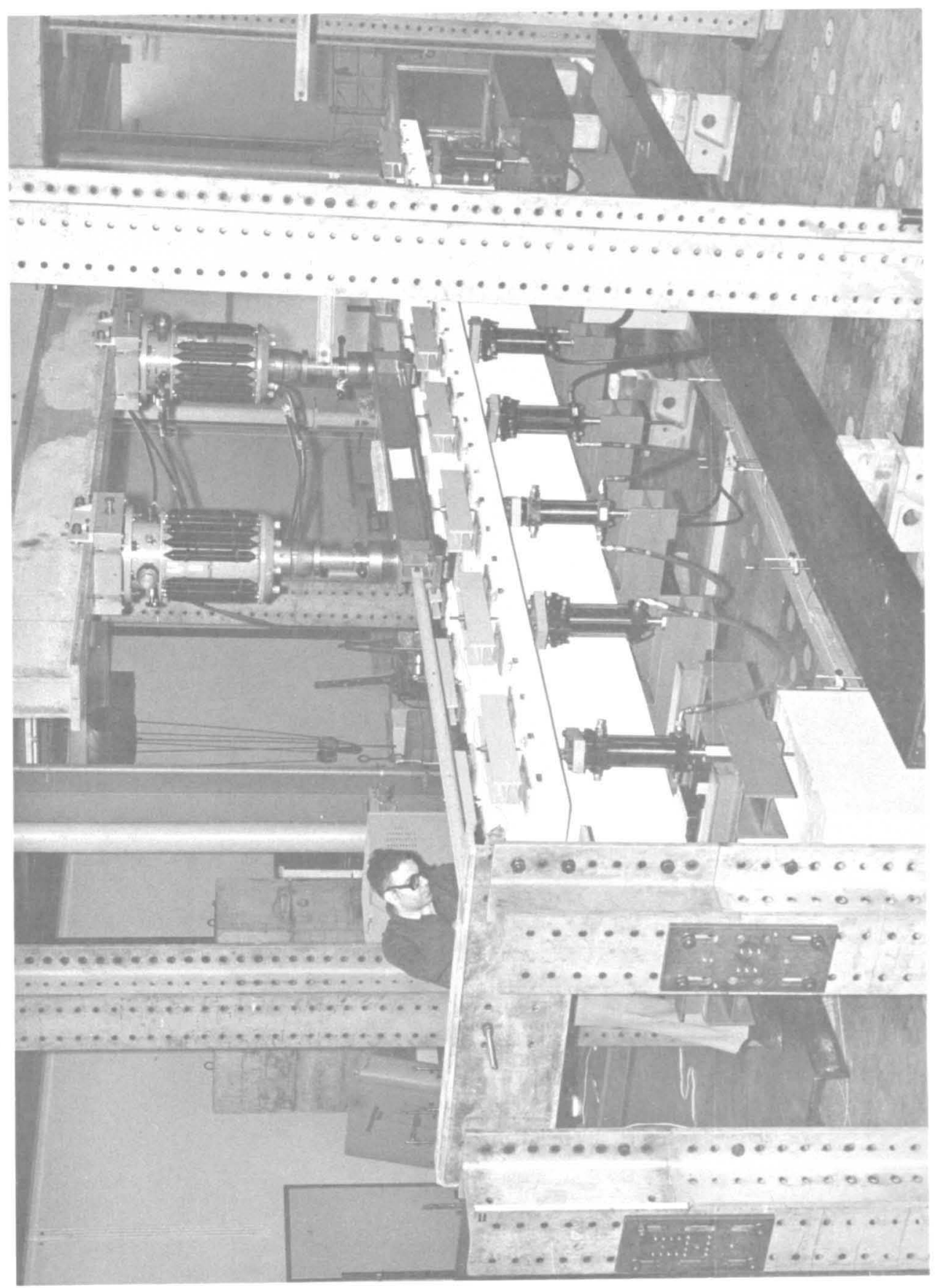
Loststellung $P$ für Löngsbeanspruchung

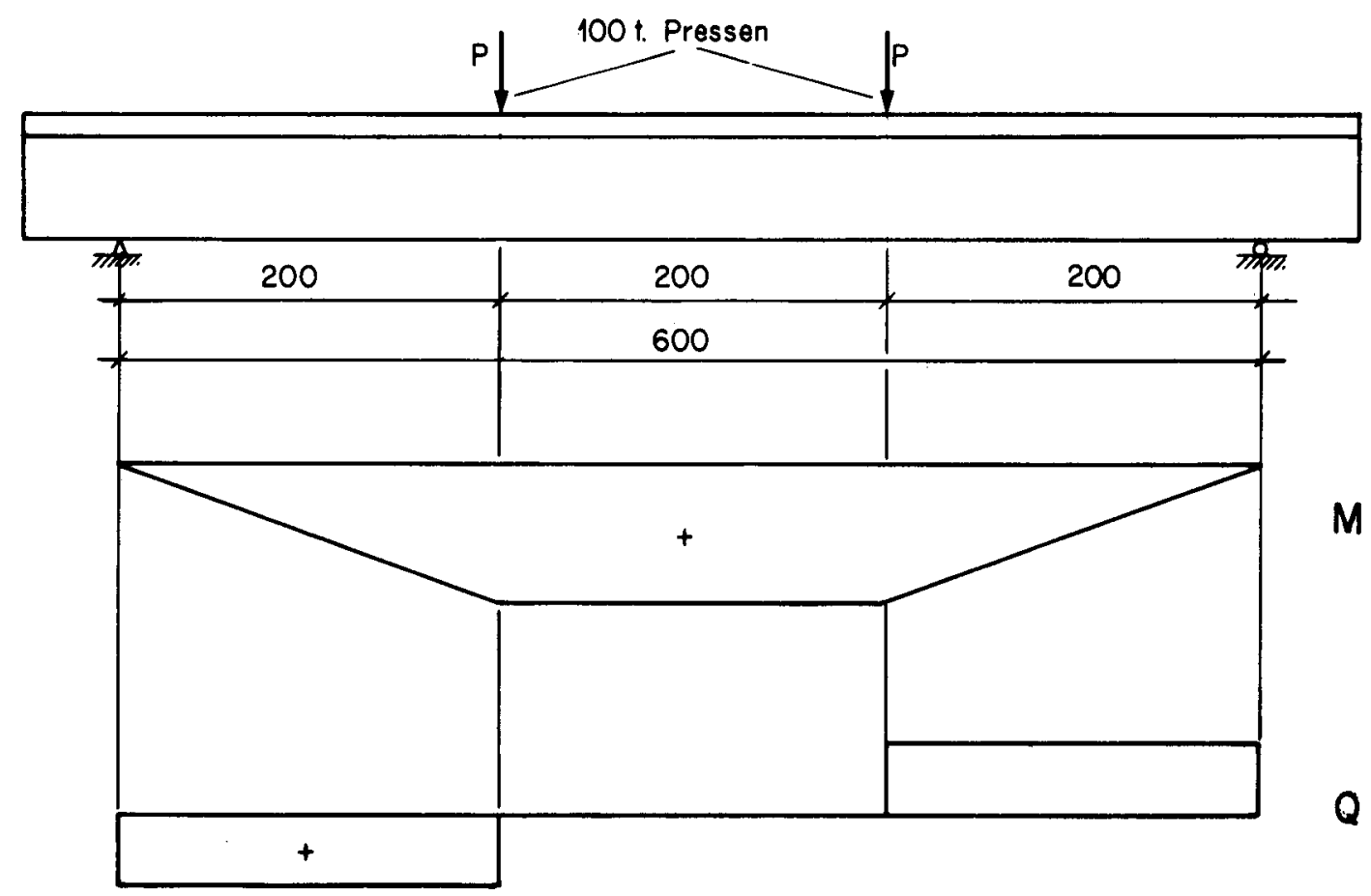

Loststellung $P$ für Querbeanspruchung
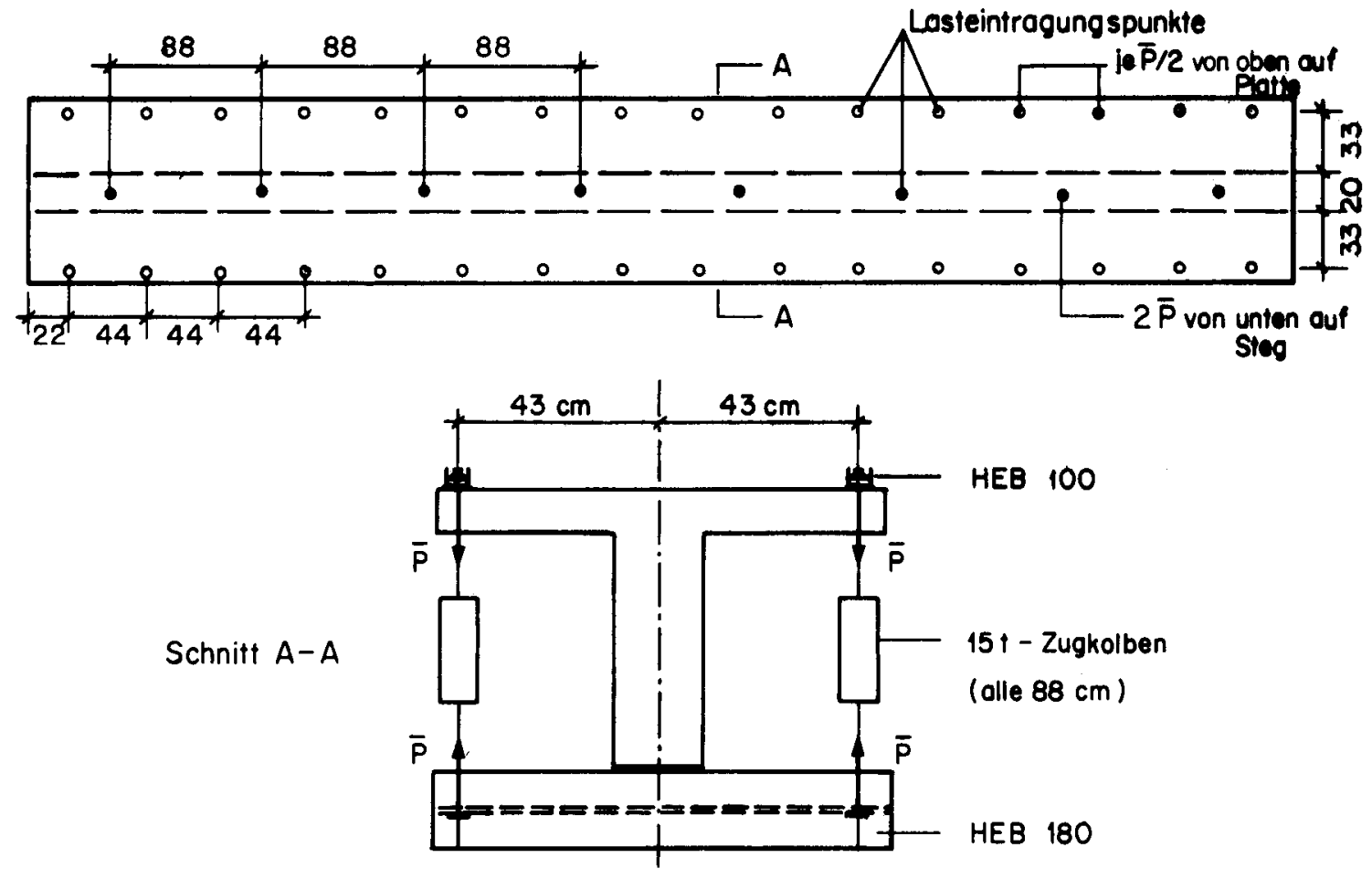

Bild 13: Belastungsanordnung für Längs-und Querbeanspruchung 

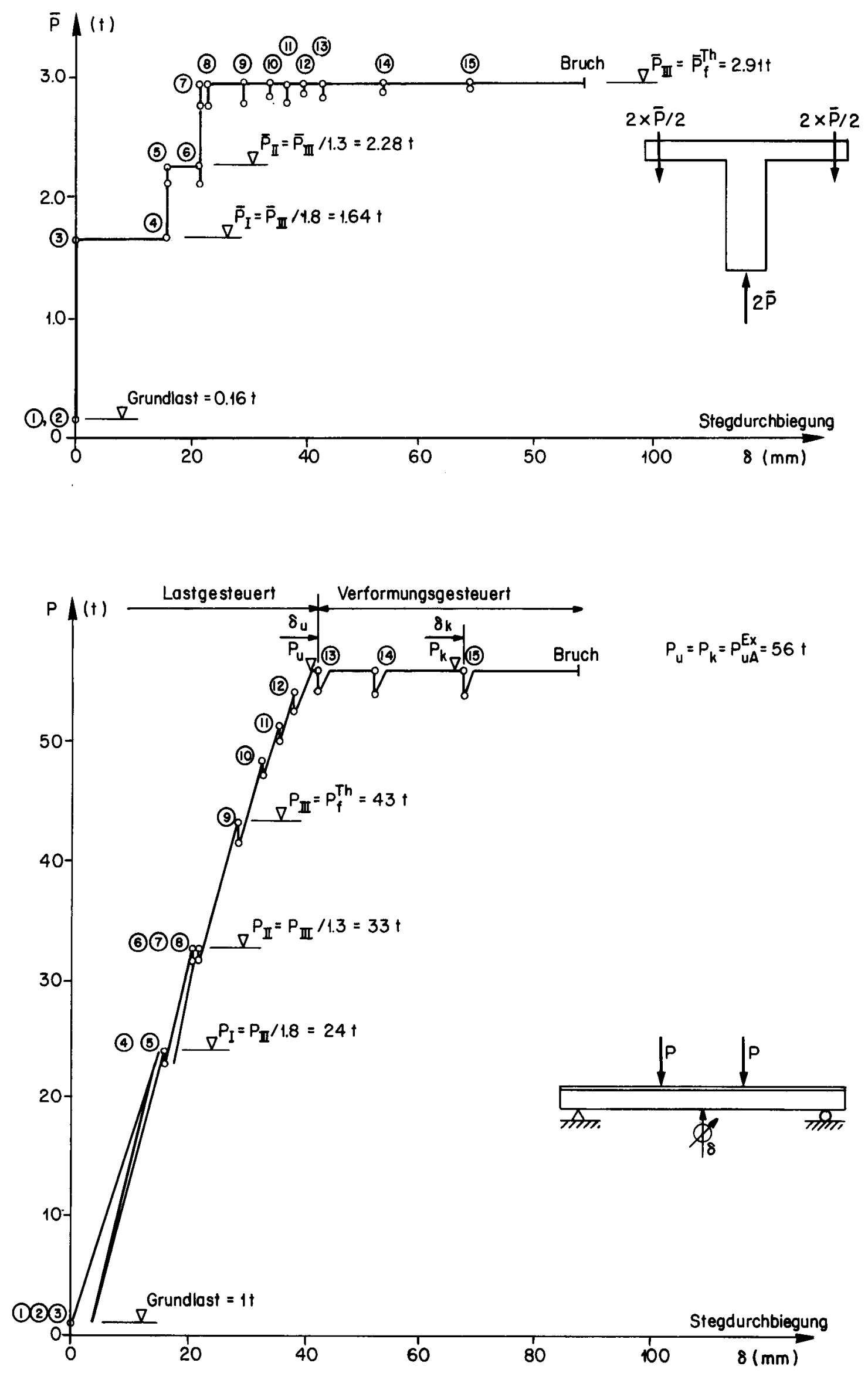

Bild 14: Belastungsablauf bei Balken Q 6 

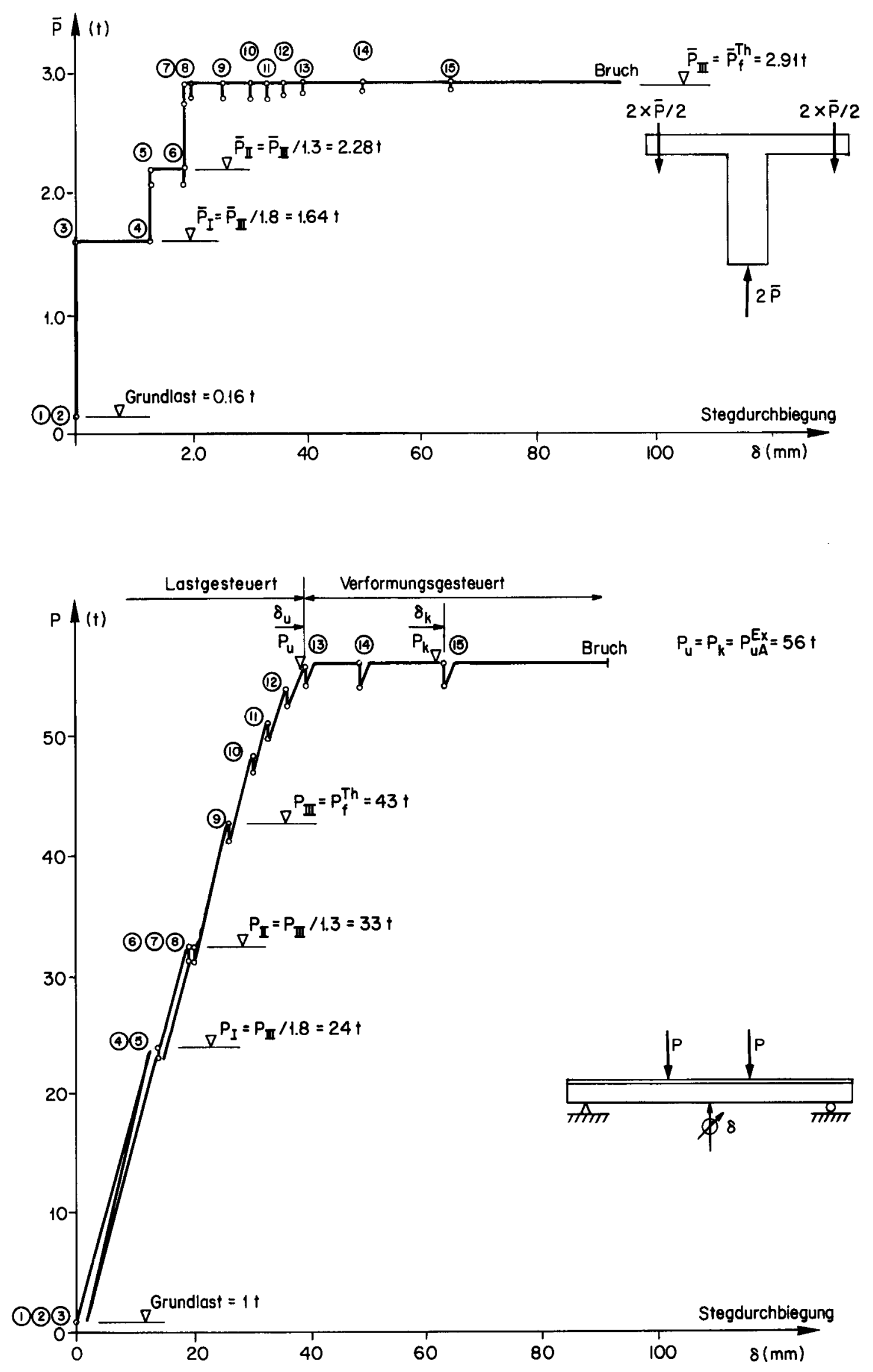

Bild 15: Belastungsablauf bei Balken $Q 7$ 

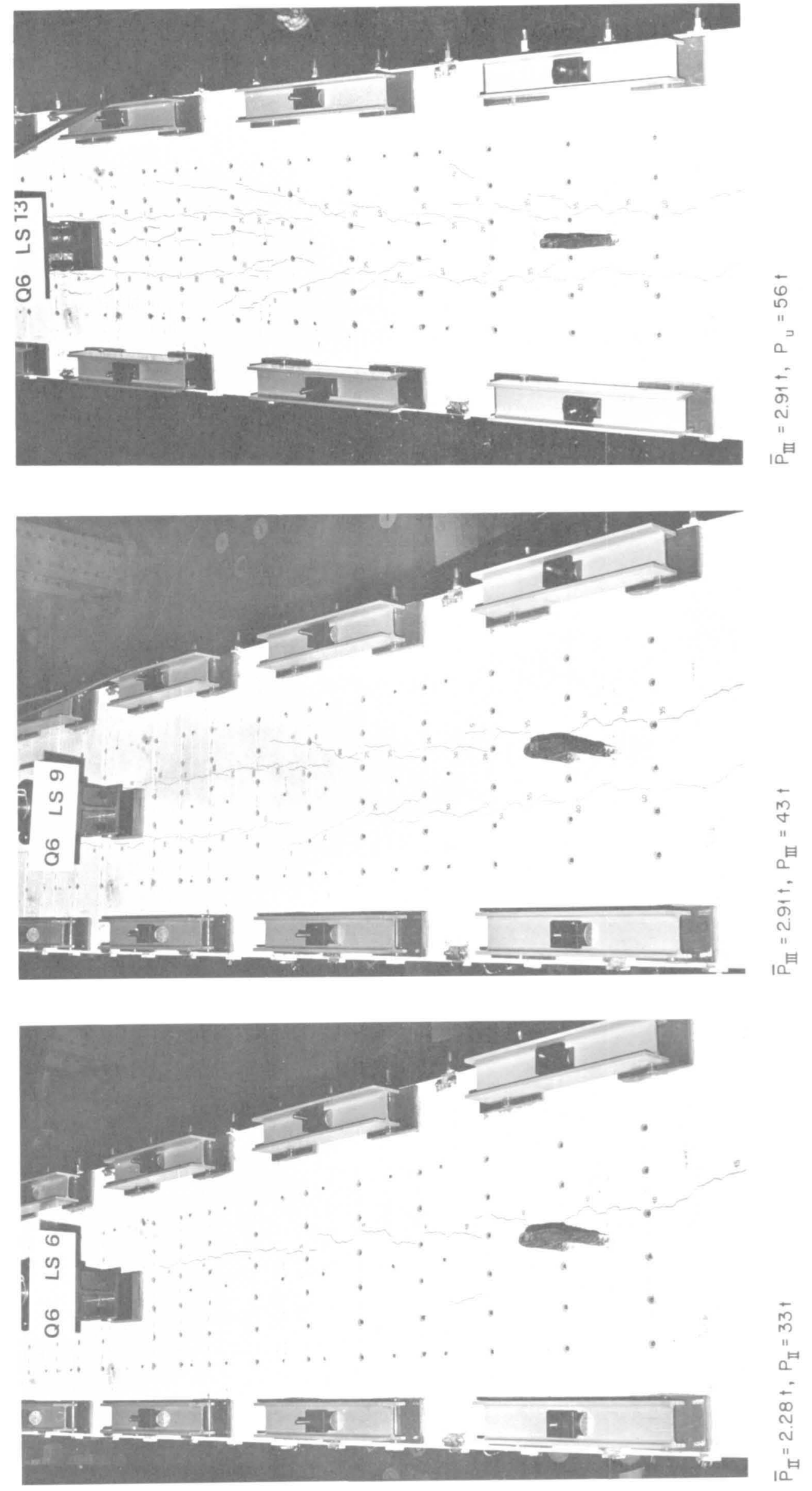


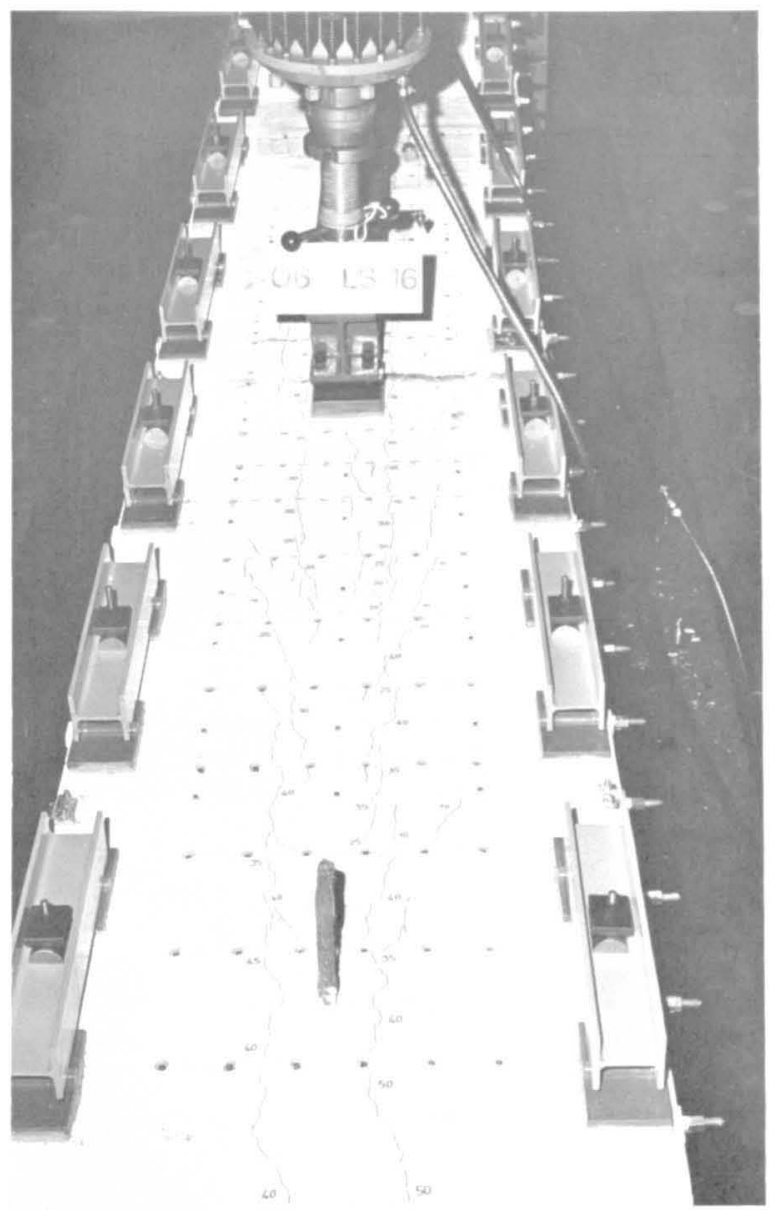

$\bar{P}_{\text {III }}=2.91 \dagger, P_{k}=56 \dagger$

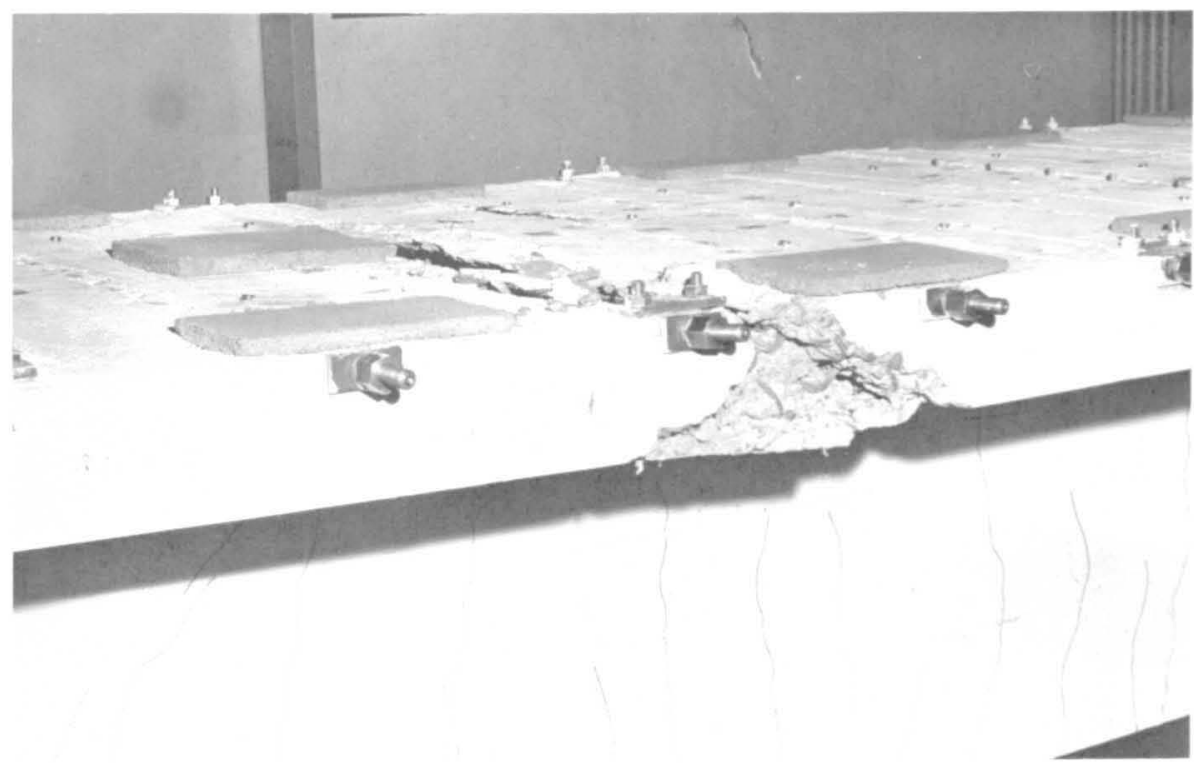

Bild 17: Bruchbilder der Platte bei Balken Q 6 

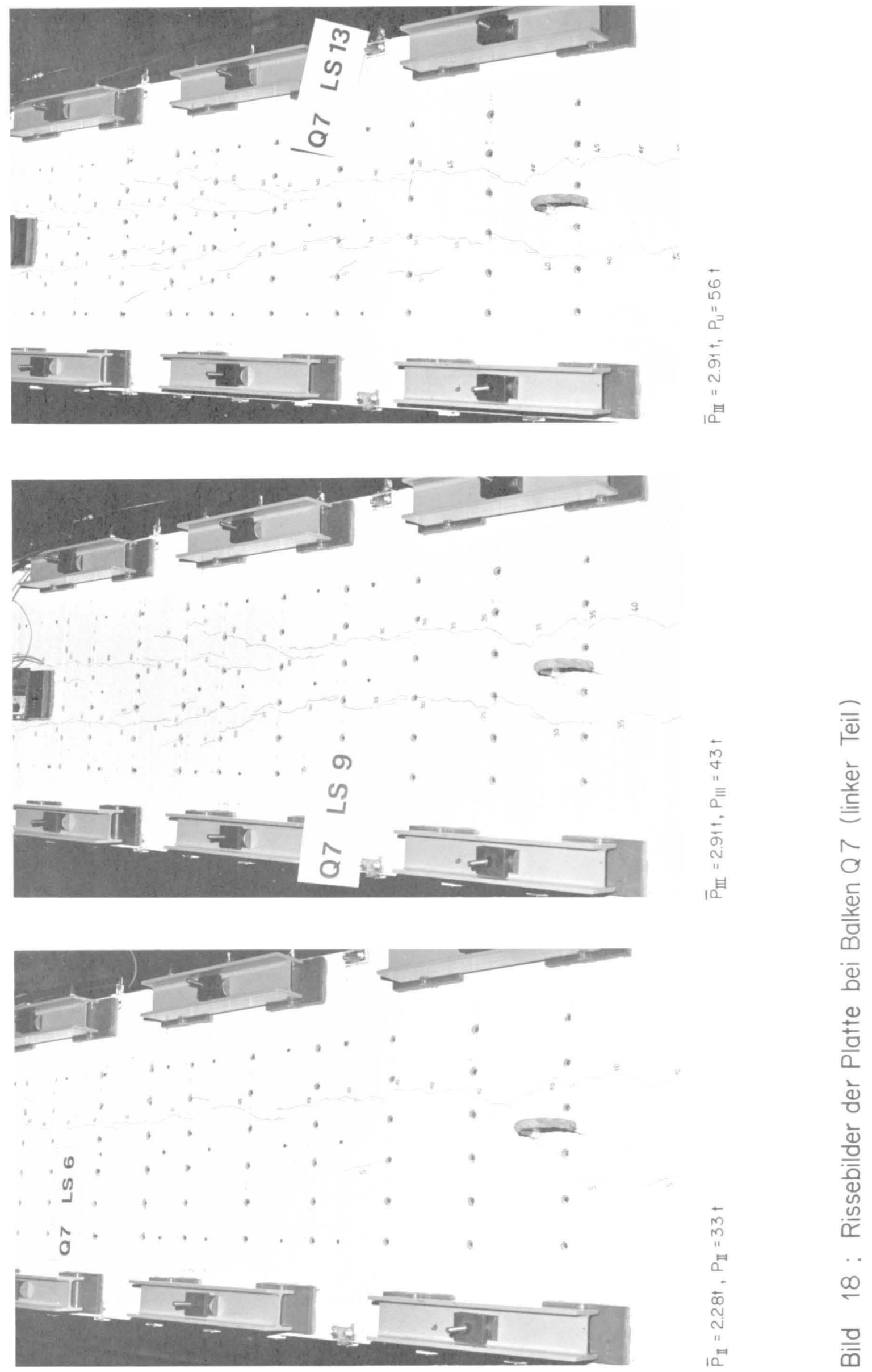


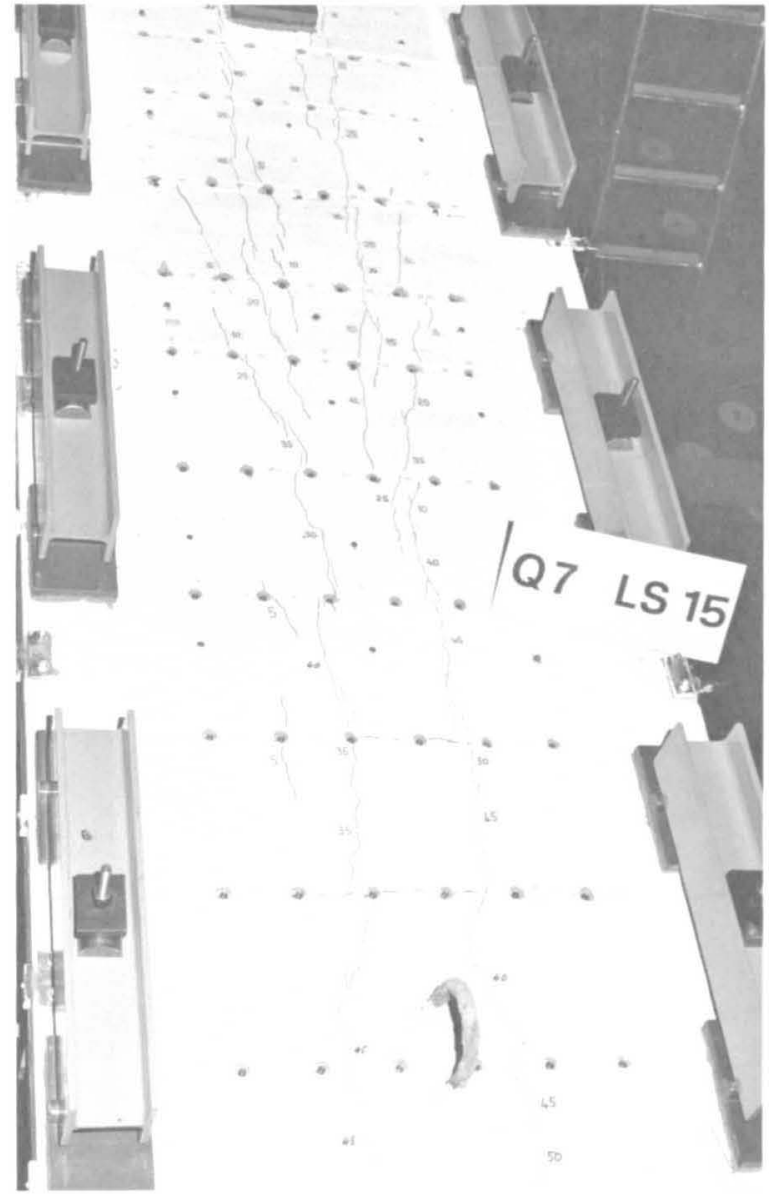

$\bar{P}_{\text {III }}=2.91 \dagger, P_{k}=56 \dagger$
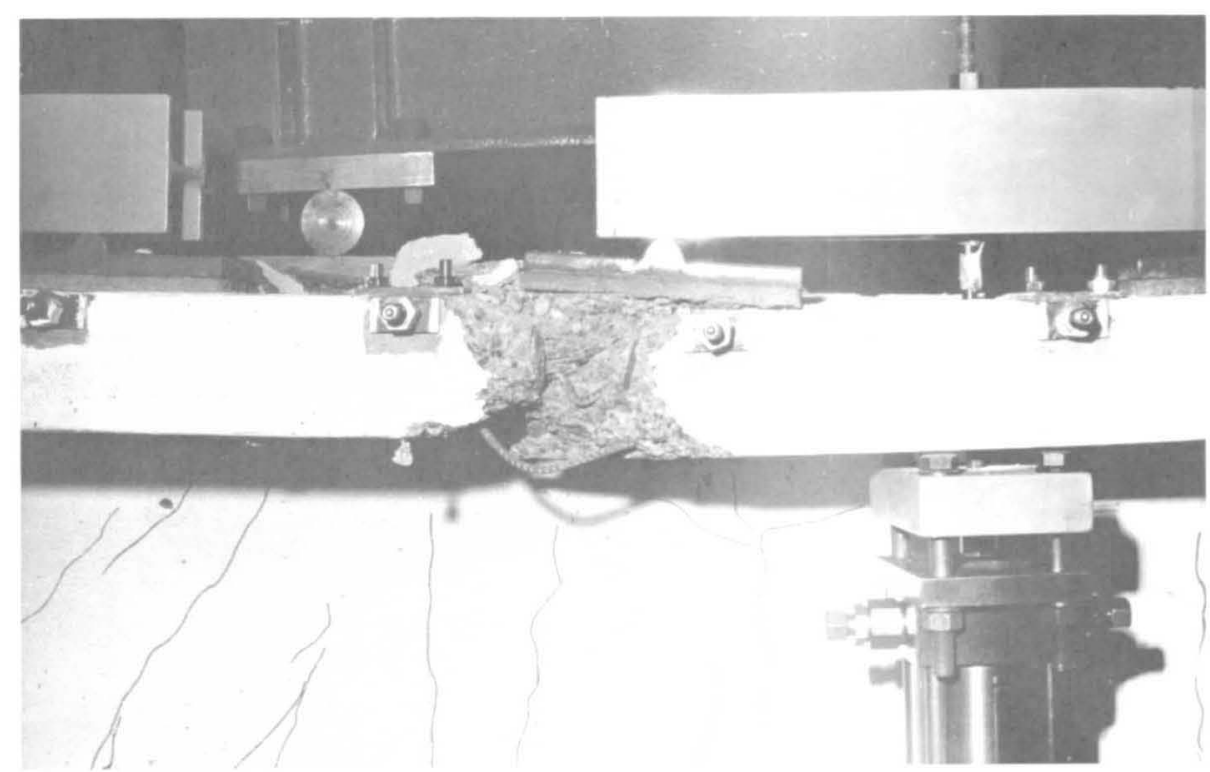

Bild 19: Bruchbilder der Platte bei Balken Q7 
Stegdurchbiegung $\delta_{\mathrm{s}}$ :
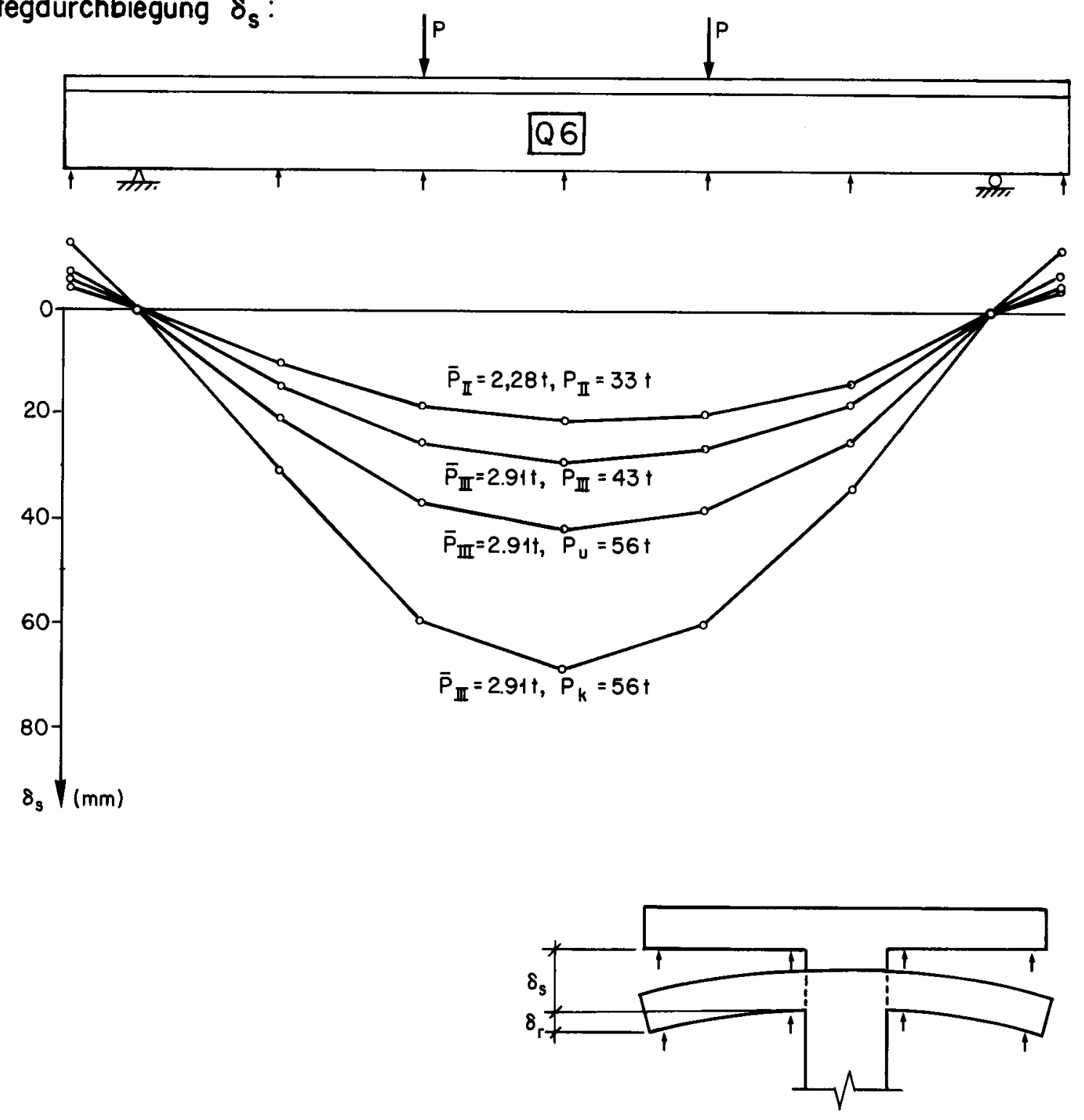

Relative Plattendurchbiegung $\delta_{\mathrm{r}}$ :

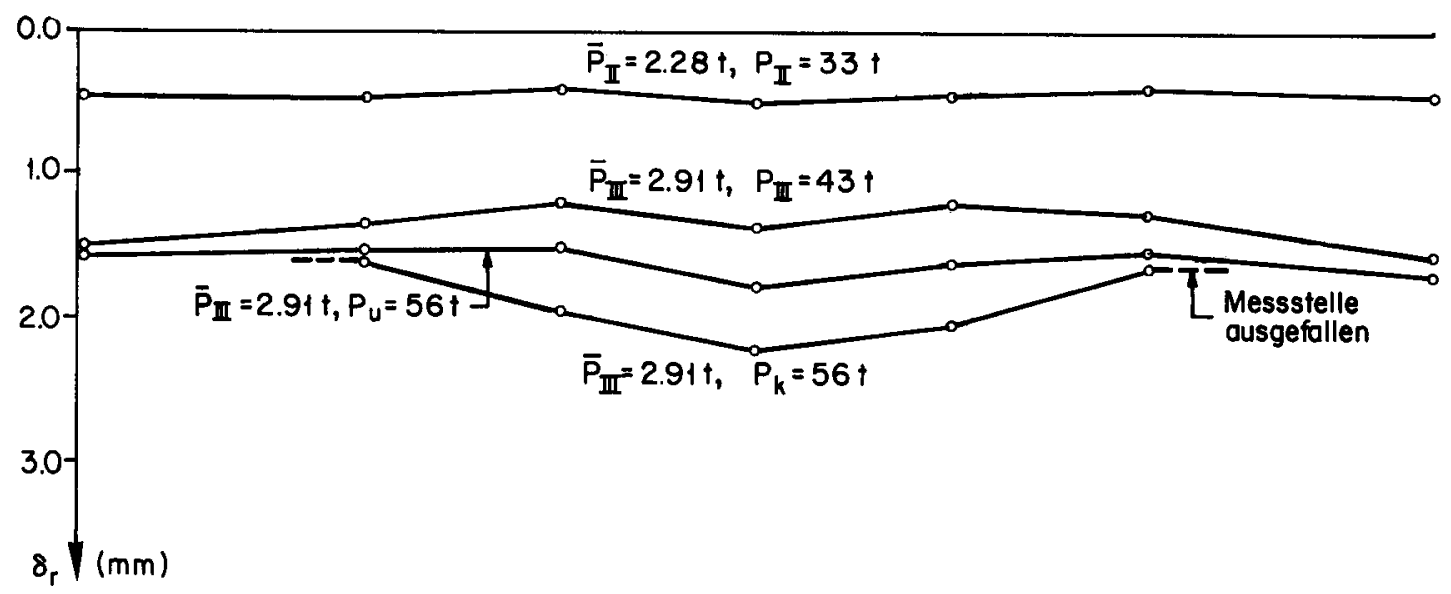

Bild 20: Durchbiegungen längs des Balkens Q6 

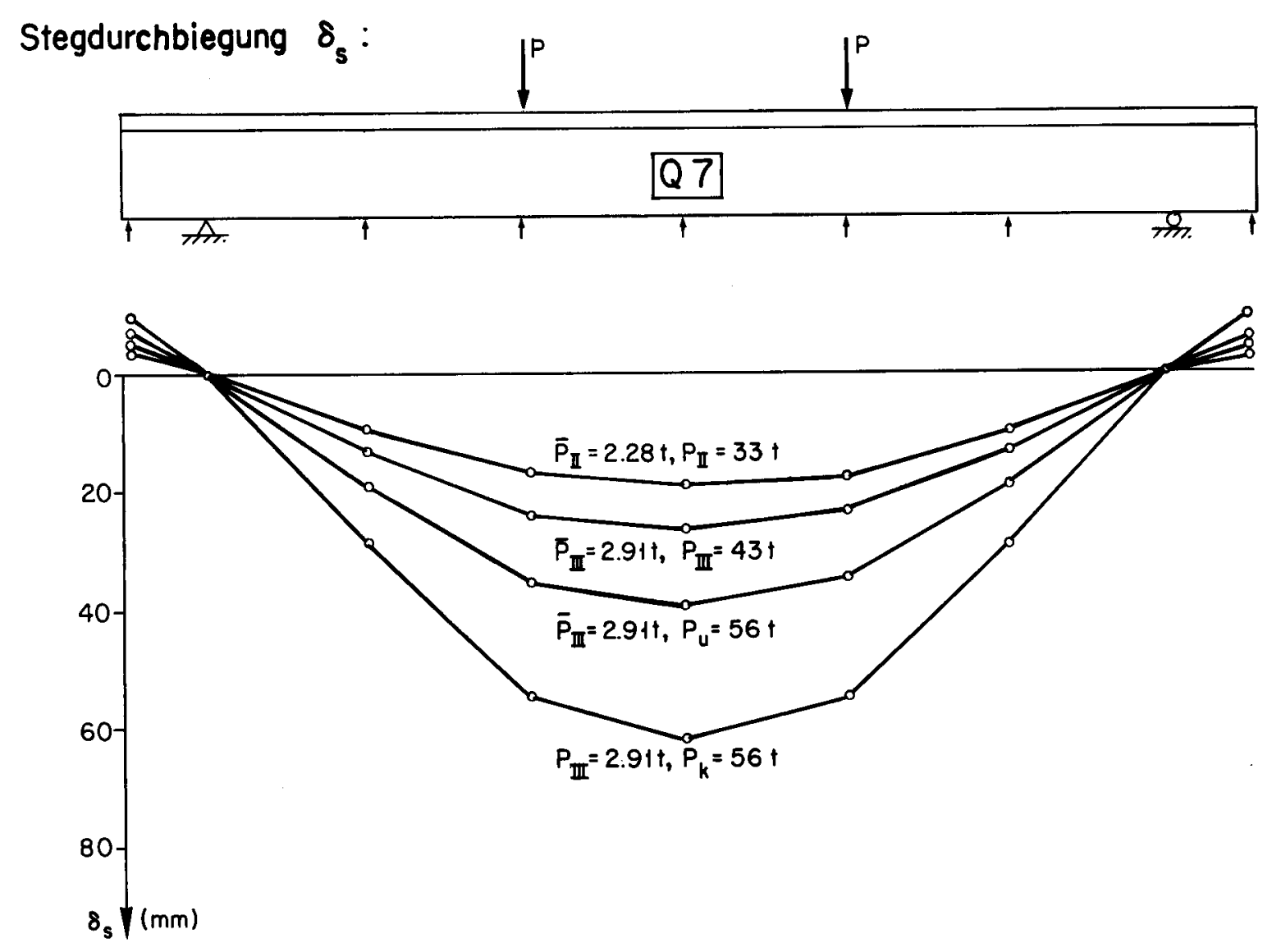

Relative Plattendurchbiegung $\delta_{\mathbf{r}}$ :

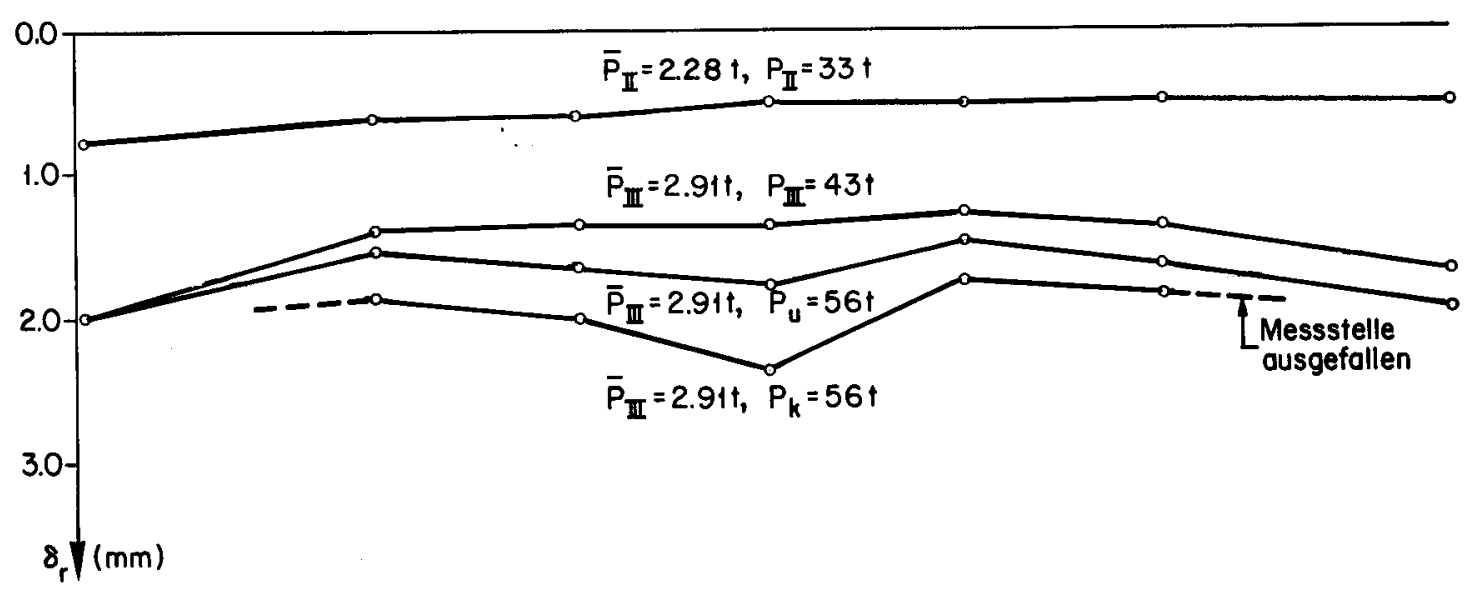

Bild 21: Durchbiegungen längs des Balkens Q7 


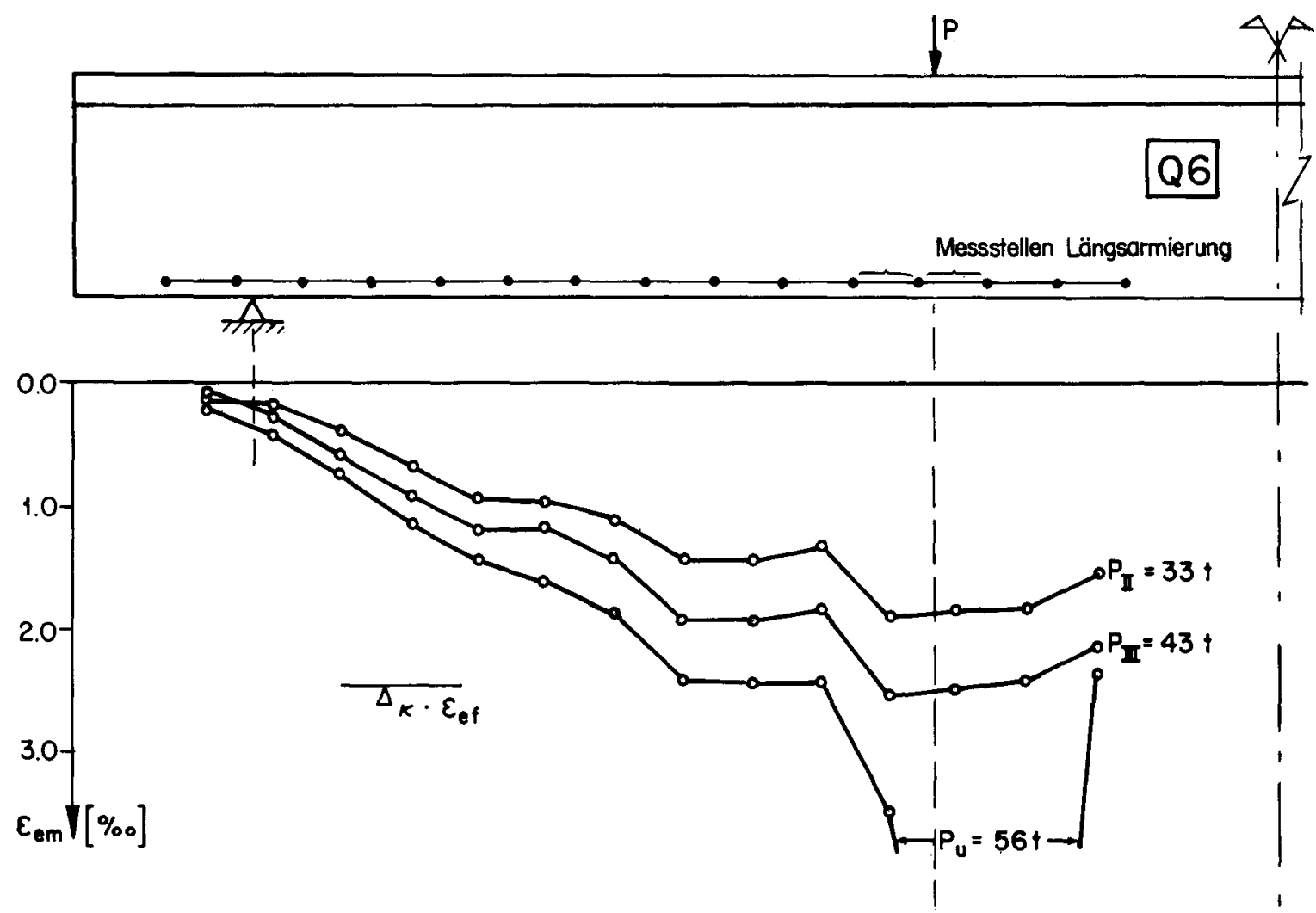

Bild 22: Dehnung der Längsarmierung bei Balken Q 6

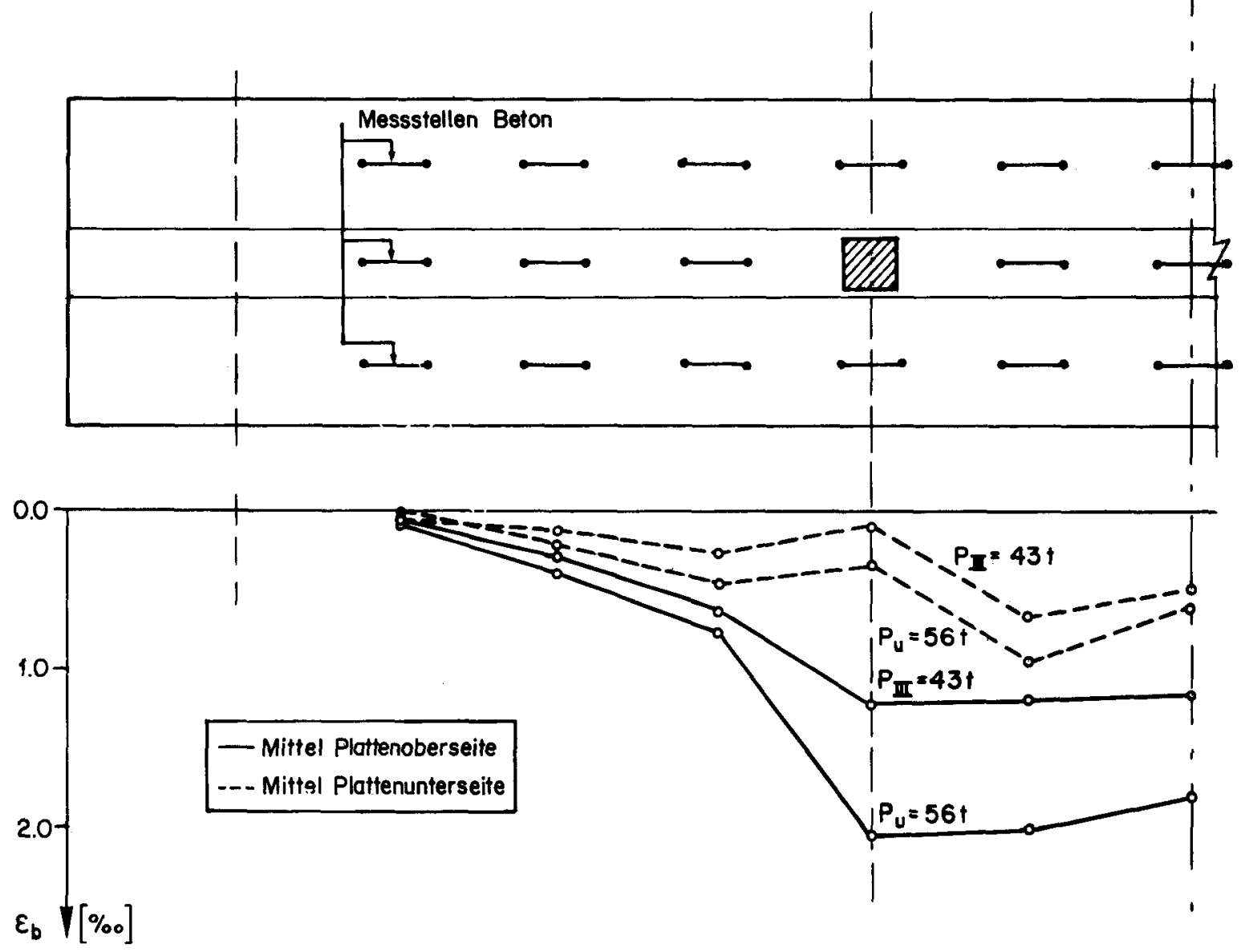

Bild 23: Betonstauchungen bei Balken $Q 6$ 


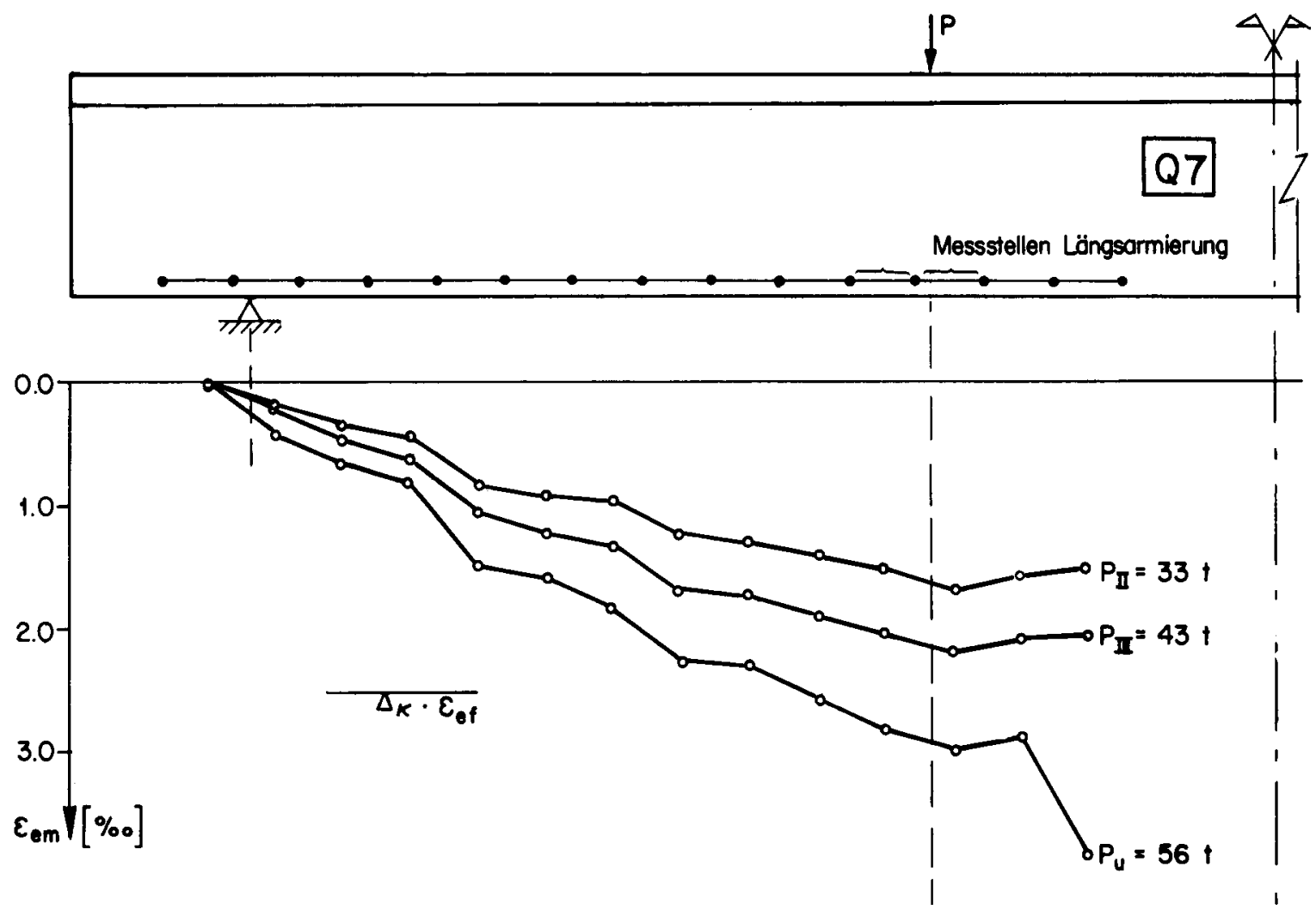

Bild 24: Dehnung der Längsarmierung bei Balken Q 7

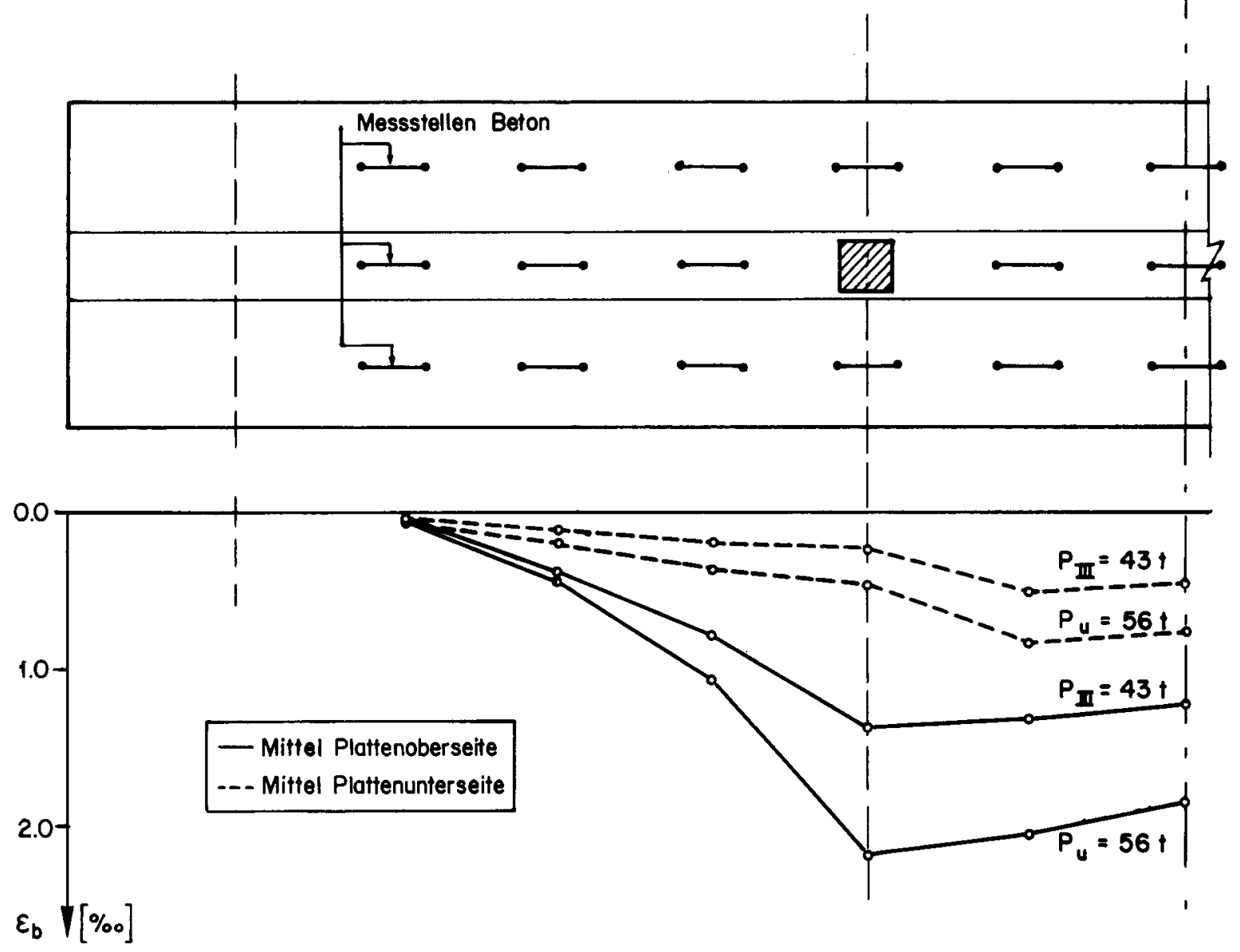

Bild 25: Betonstauchungen bei Balken Q7 


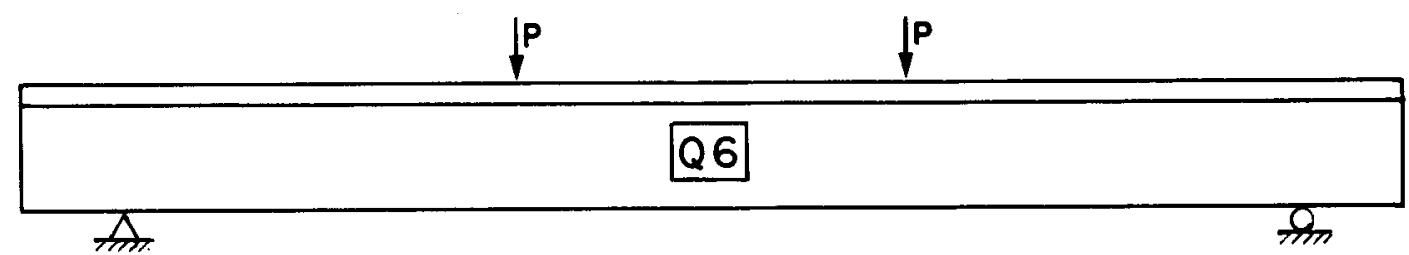

Obere Lage der Plattenarmierung

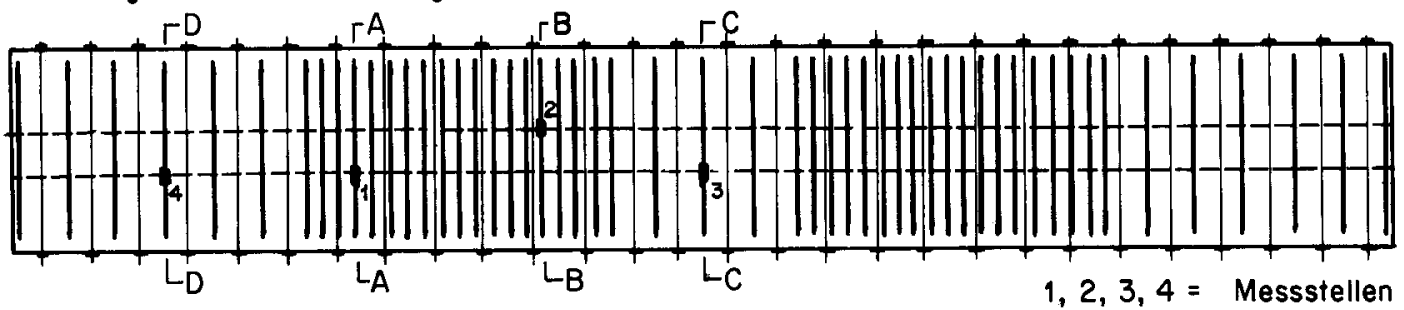

Untere Loge der Plattenarmierung
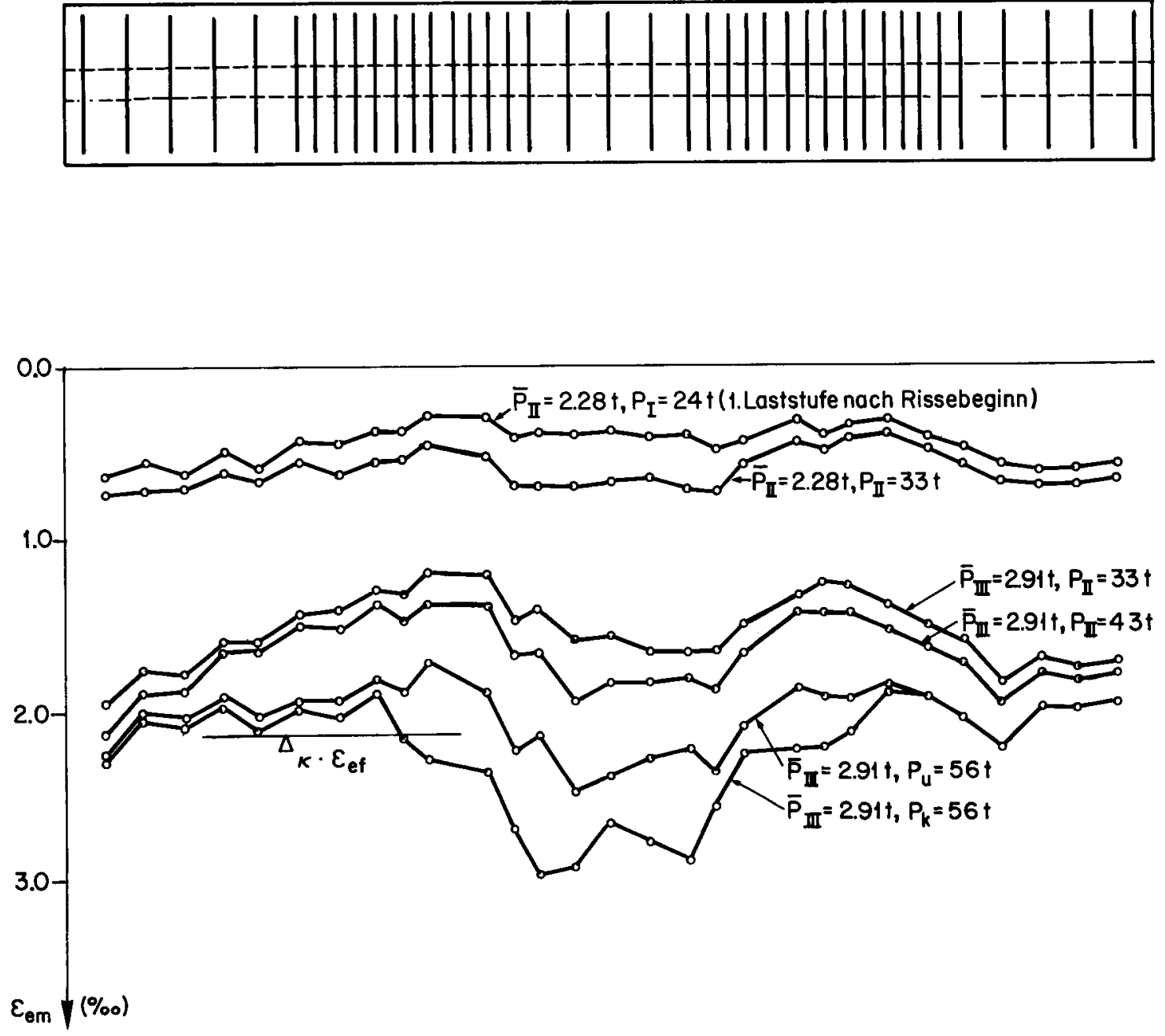

Bild 26-a: Dehnung der oberen Plattenarmierung bei Balken Q6 

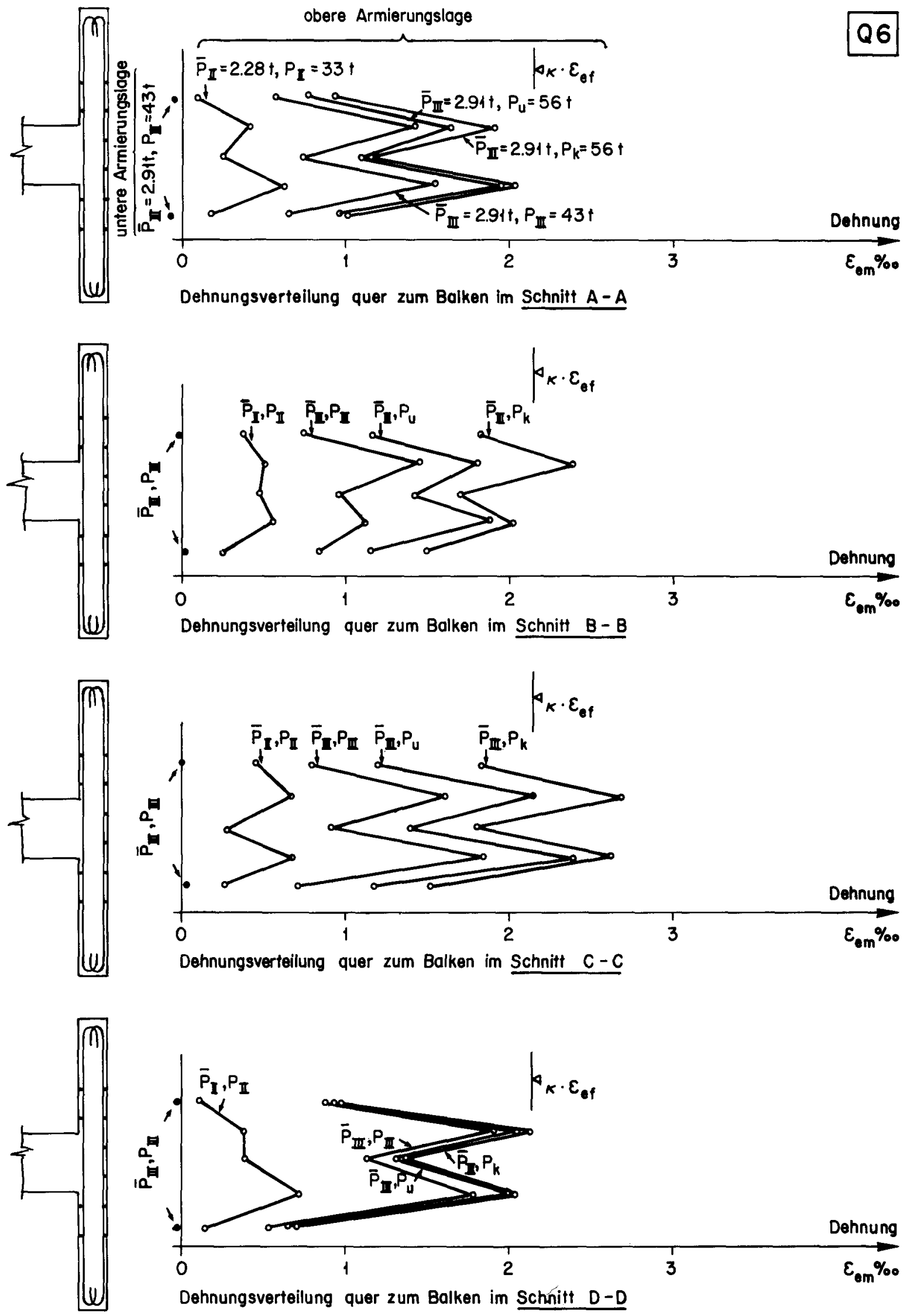

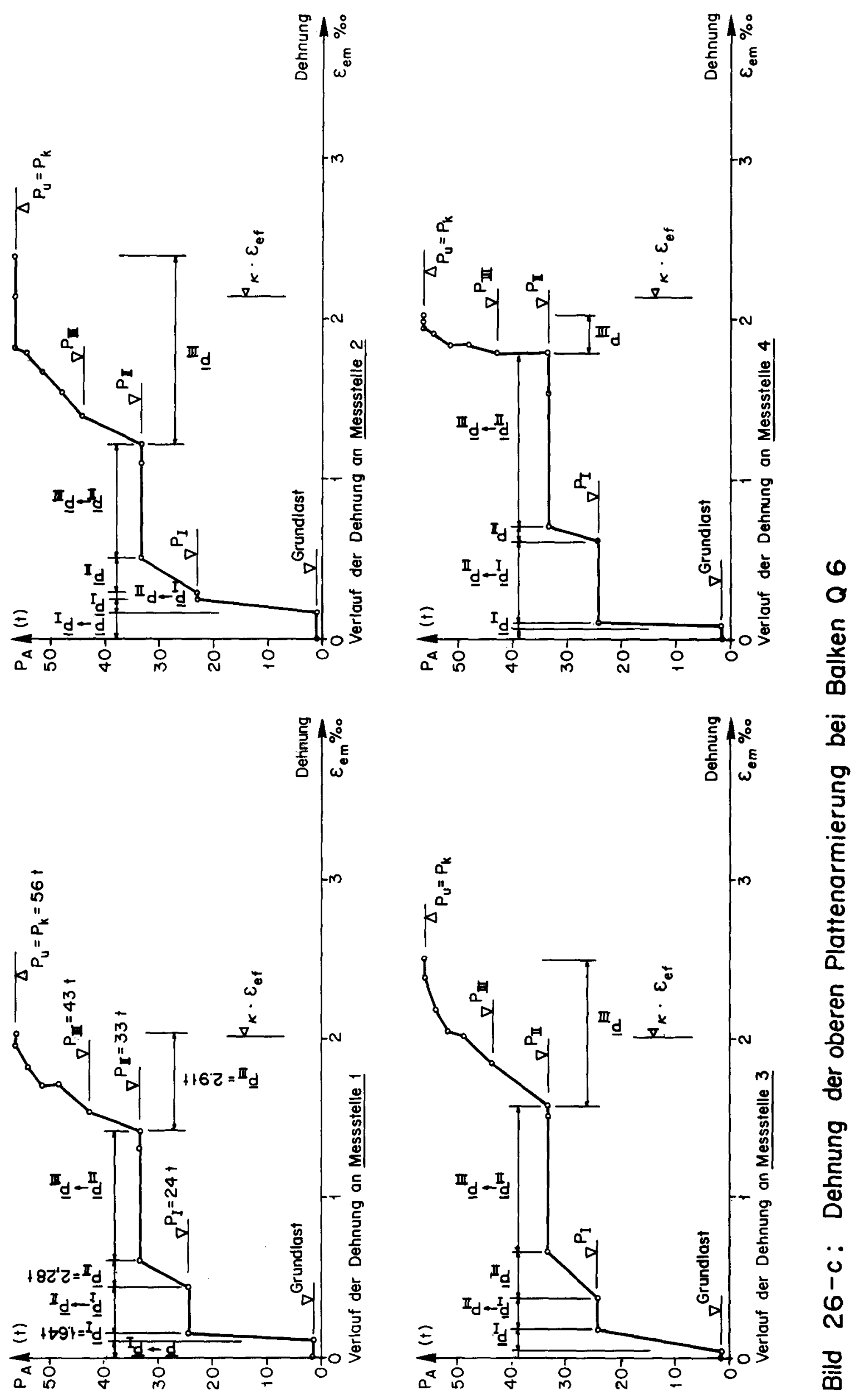


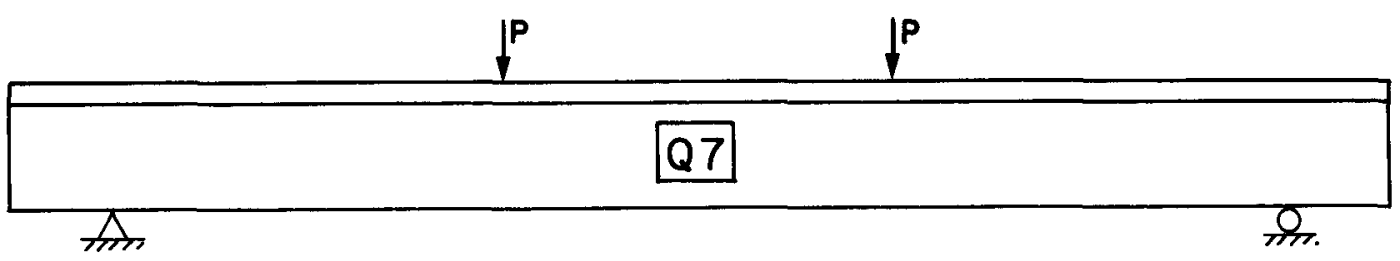

Obere Lage der Plattenarmierung

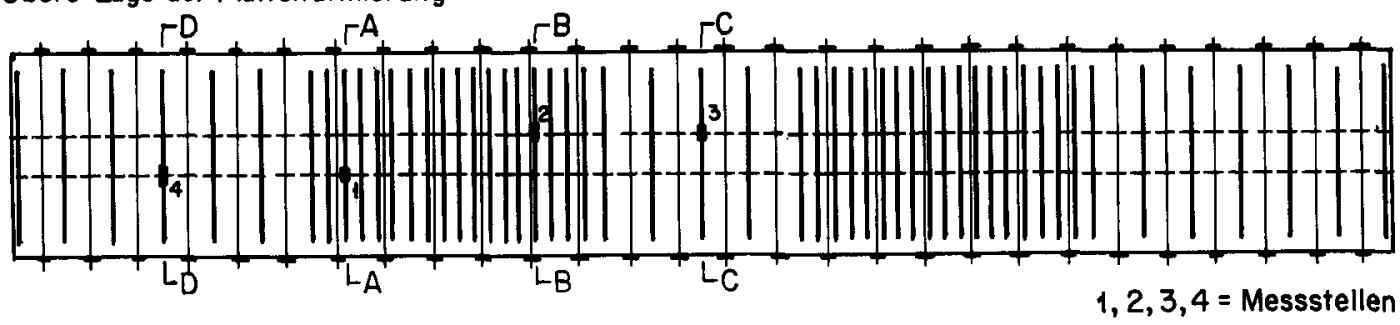

Untere Loge der Plattenarmierung
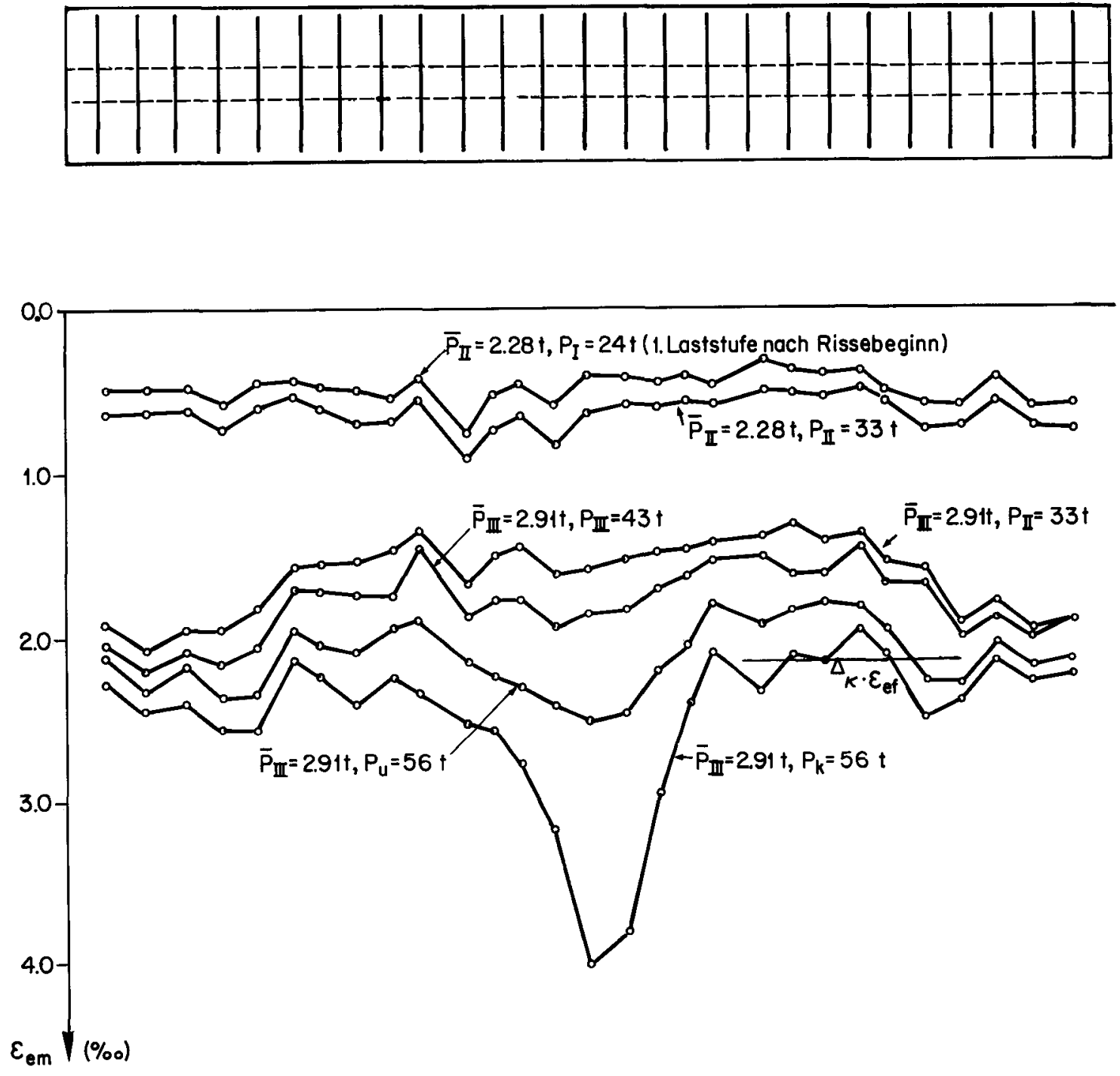

Bild 27-a: Dehnung der oberen Plattenarmierung bei Balken Q7 

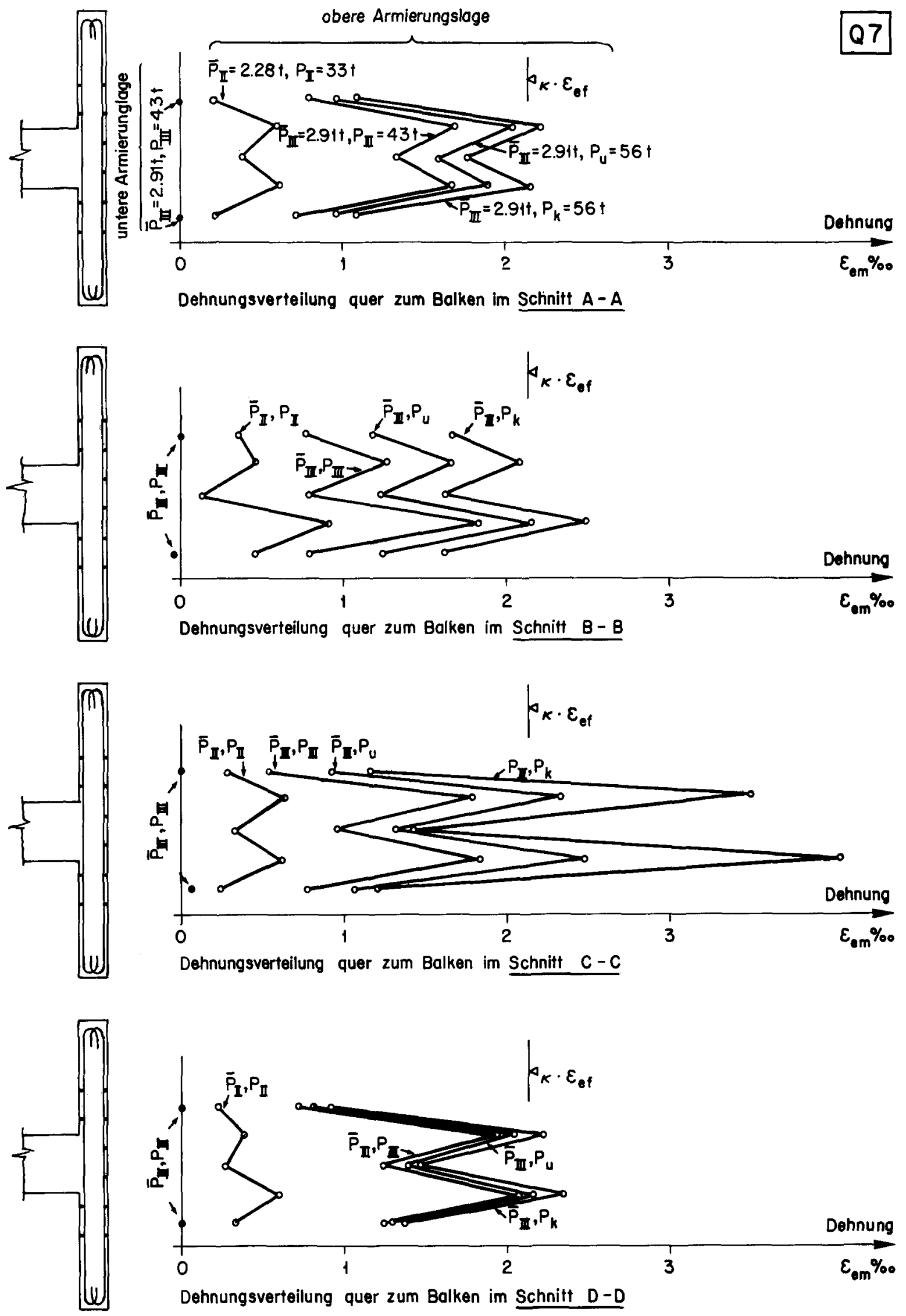

Bild 27-b: Dehnung der Plattenarmierung bei Balken Q7 

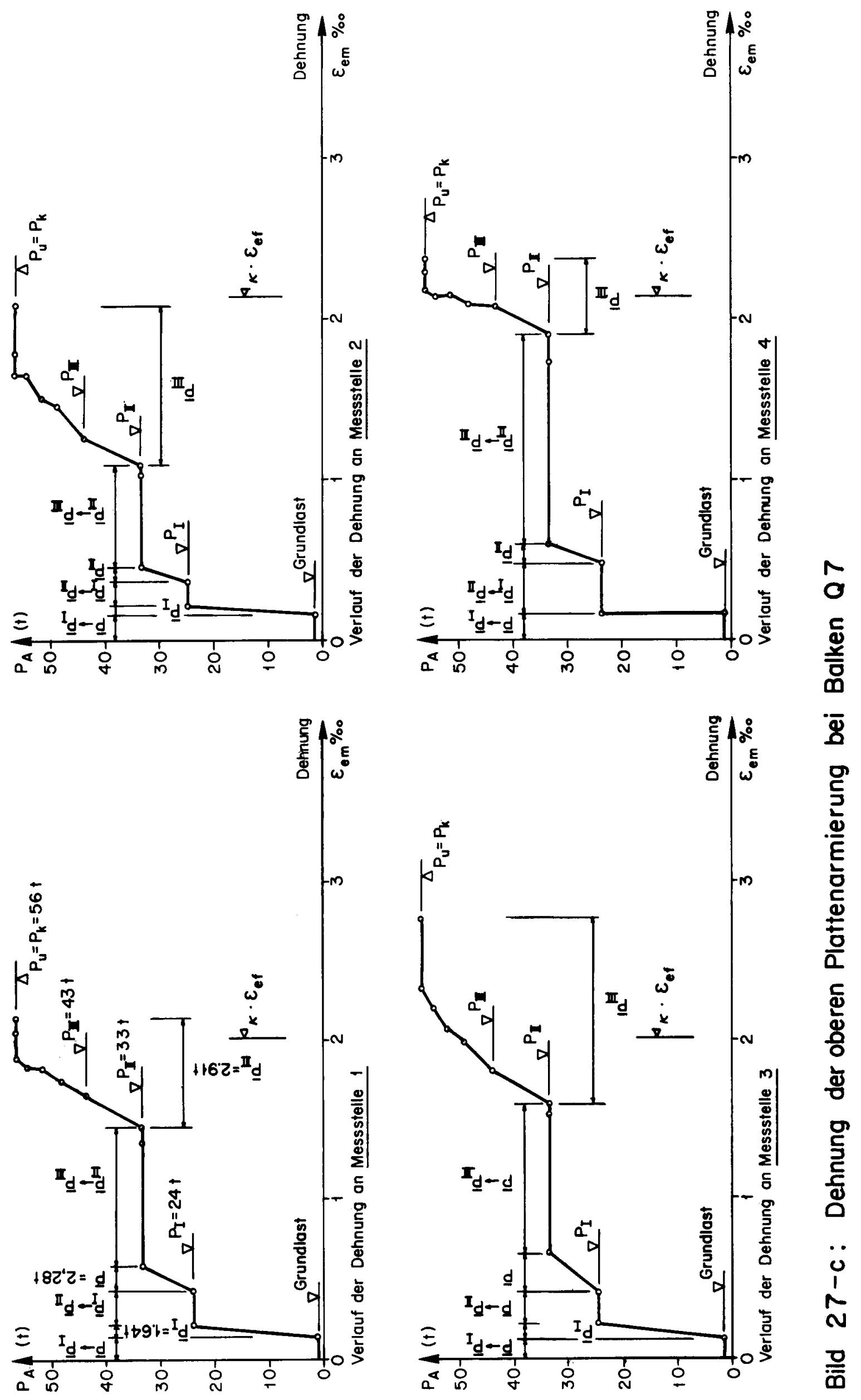

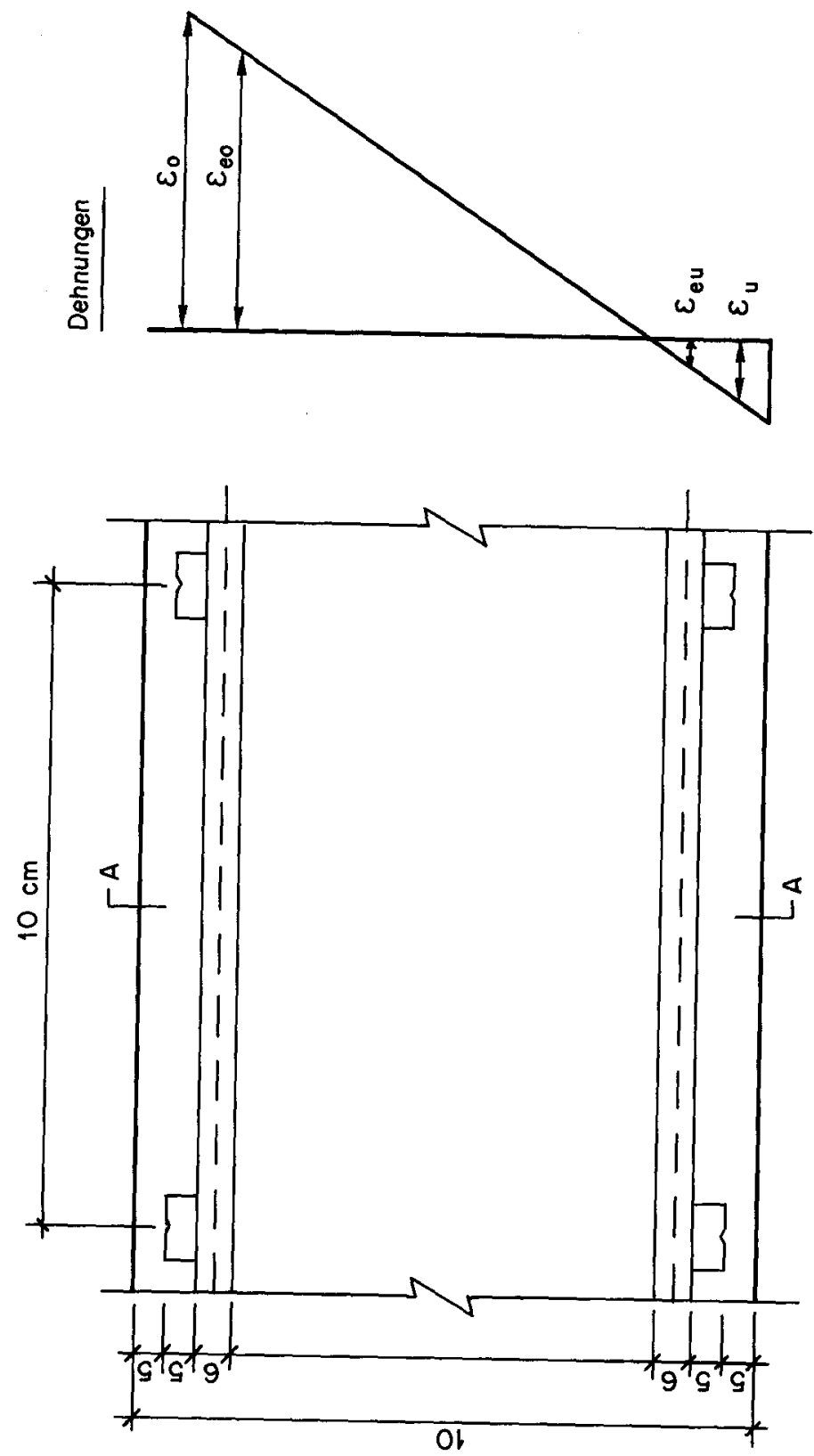

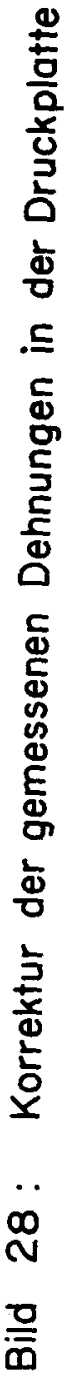




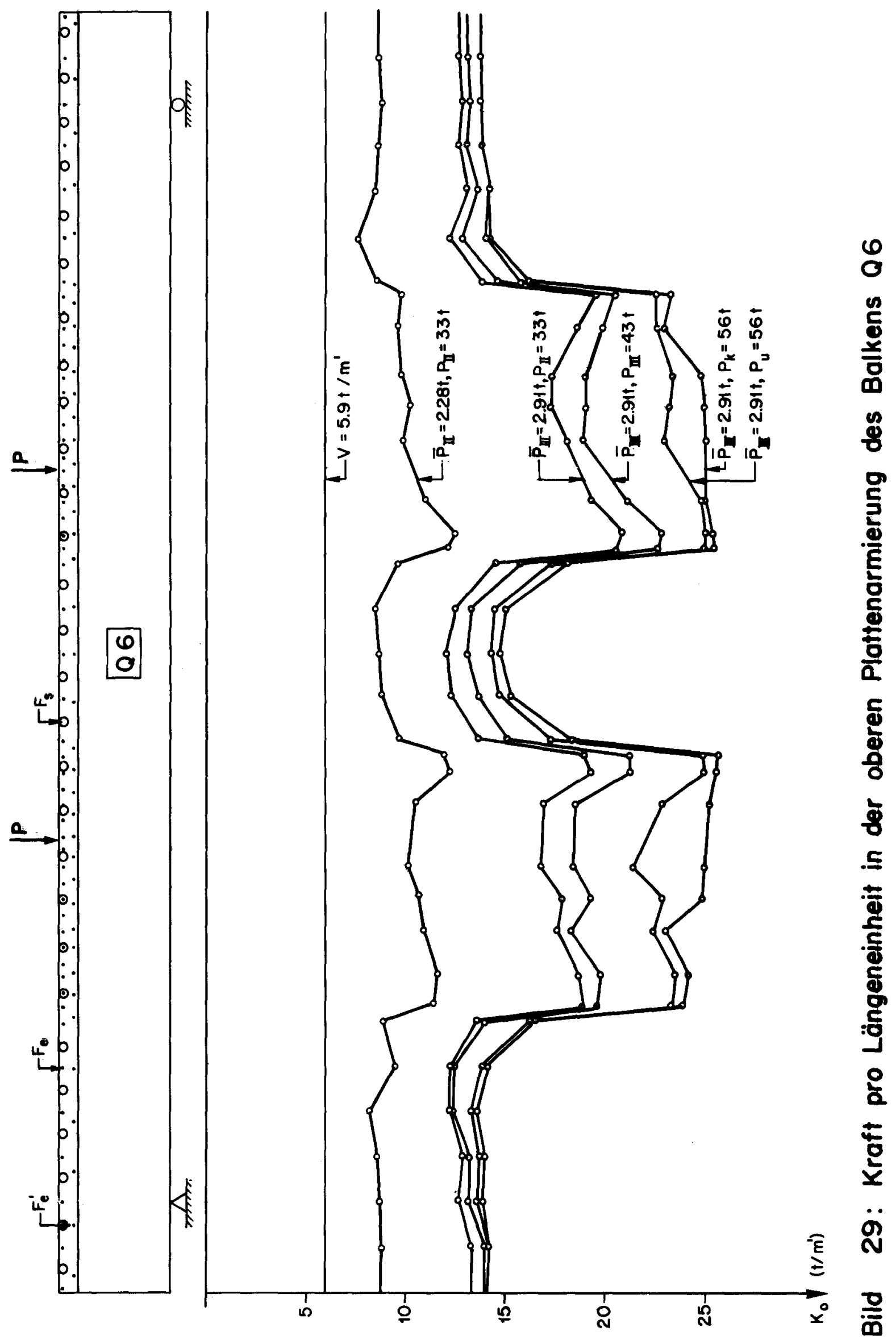




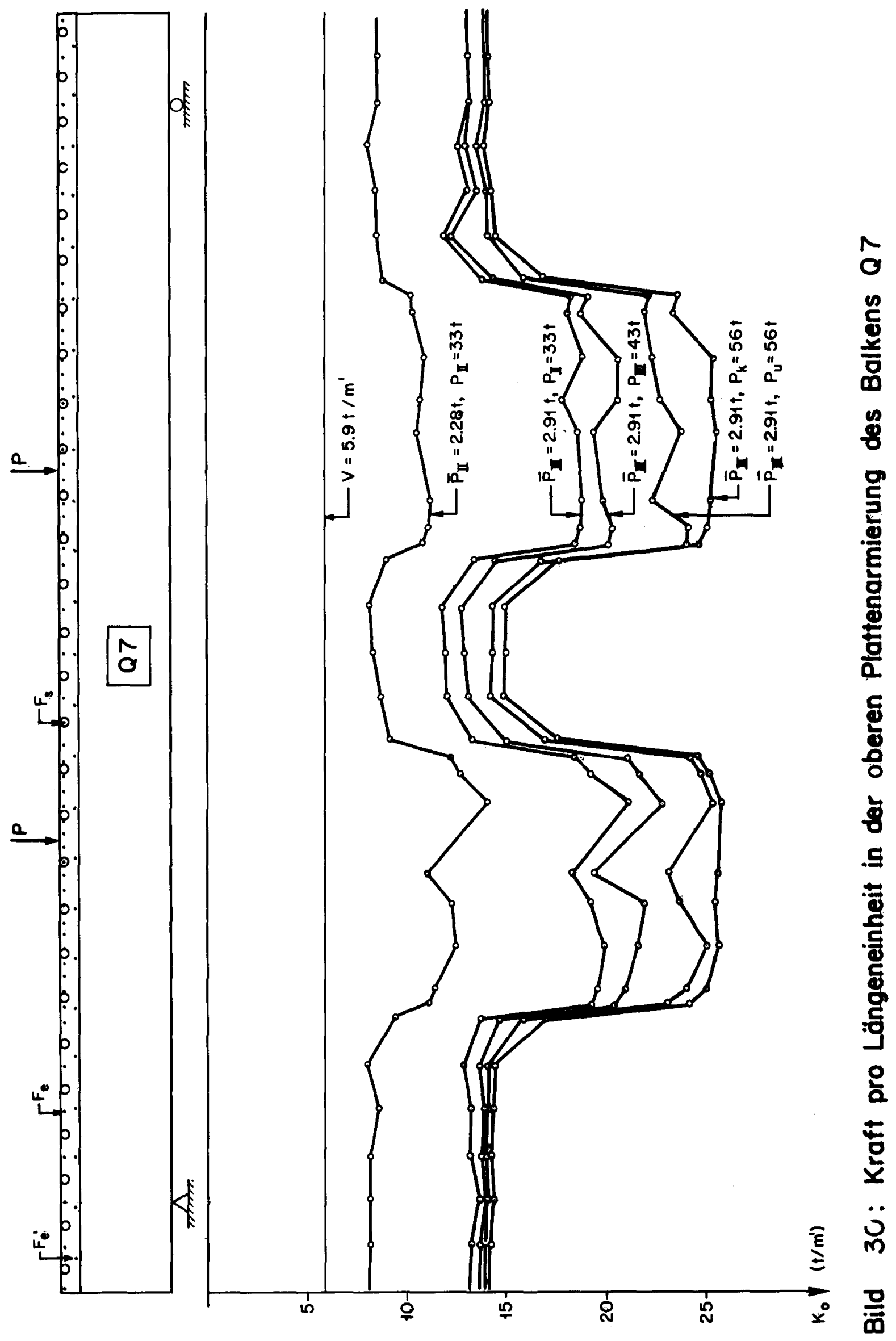




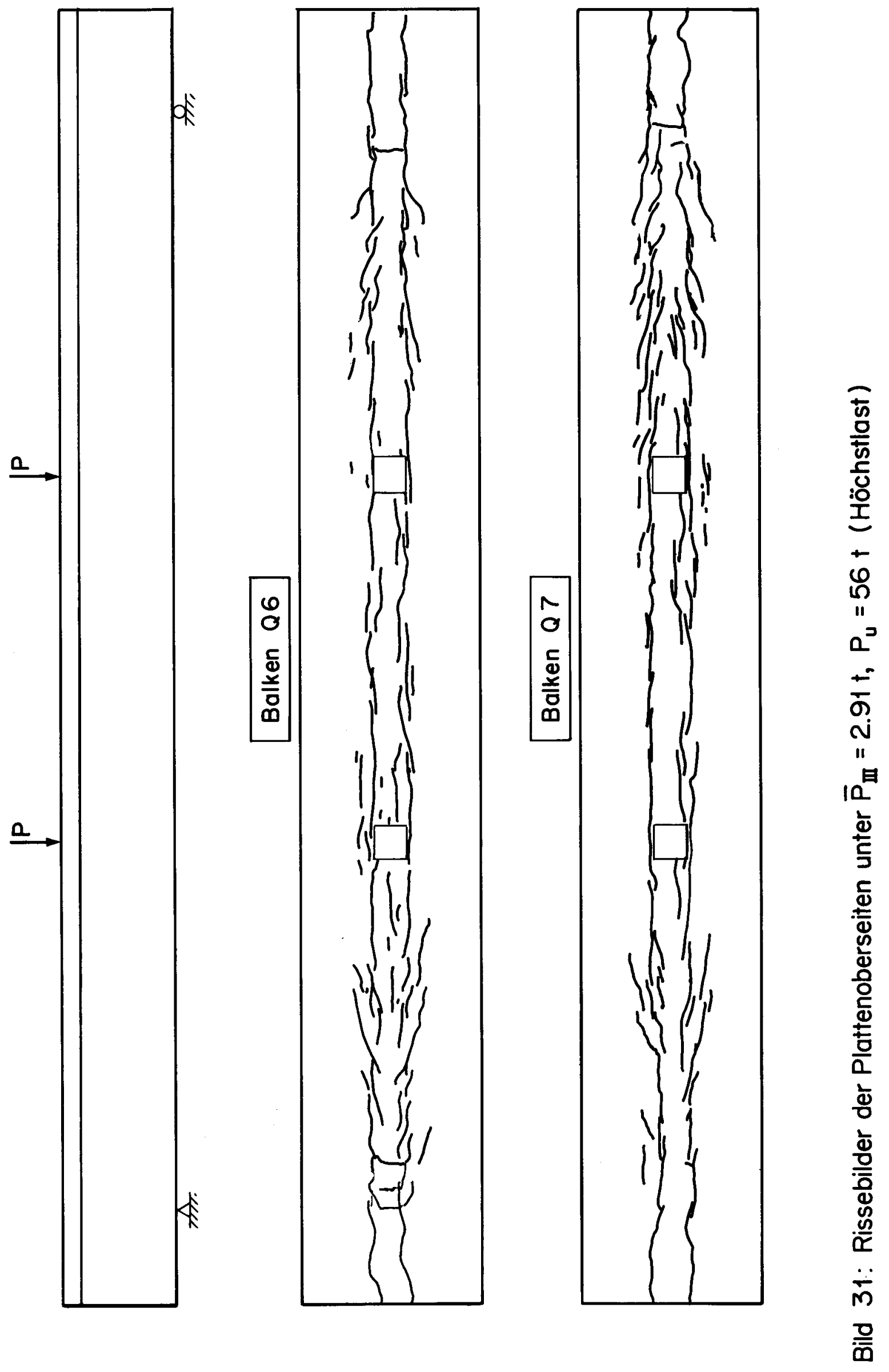




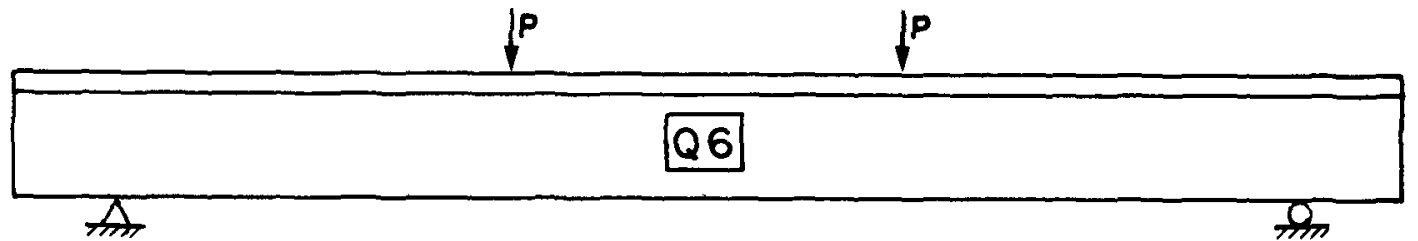

Obere Loge der Plottenarmierung

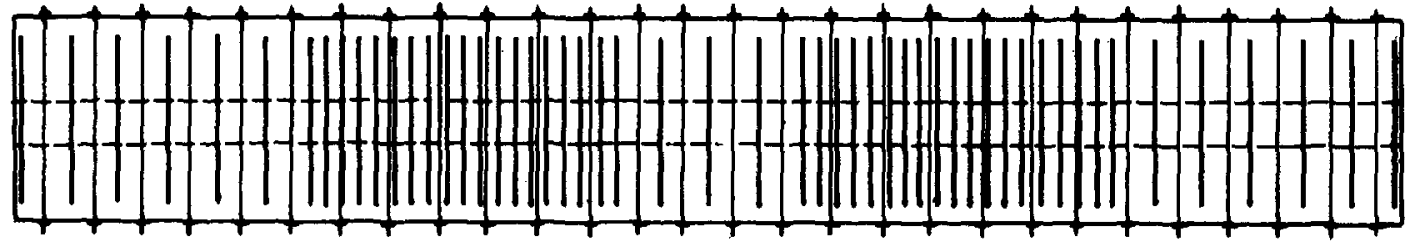

Maximale Rissbreite längs der Platte
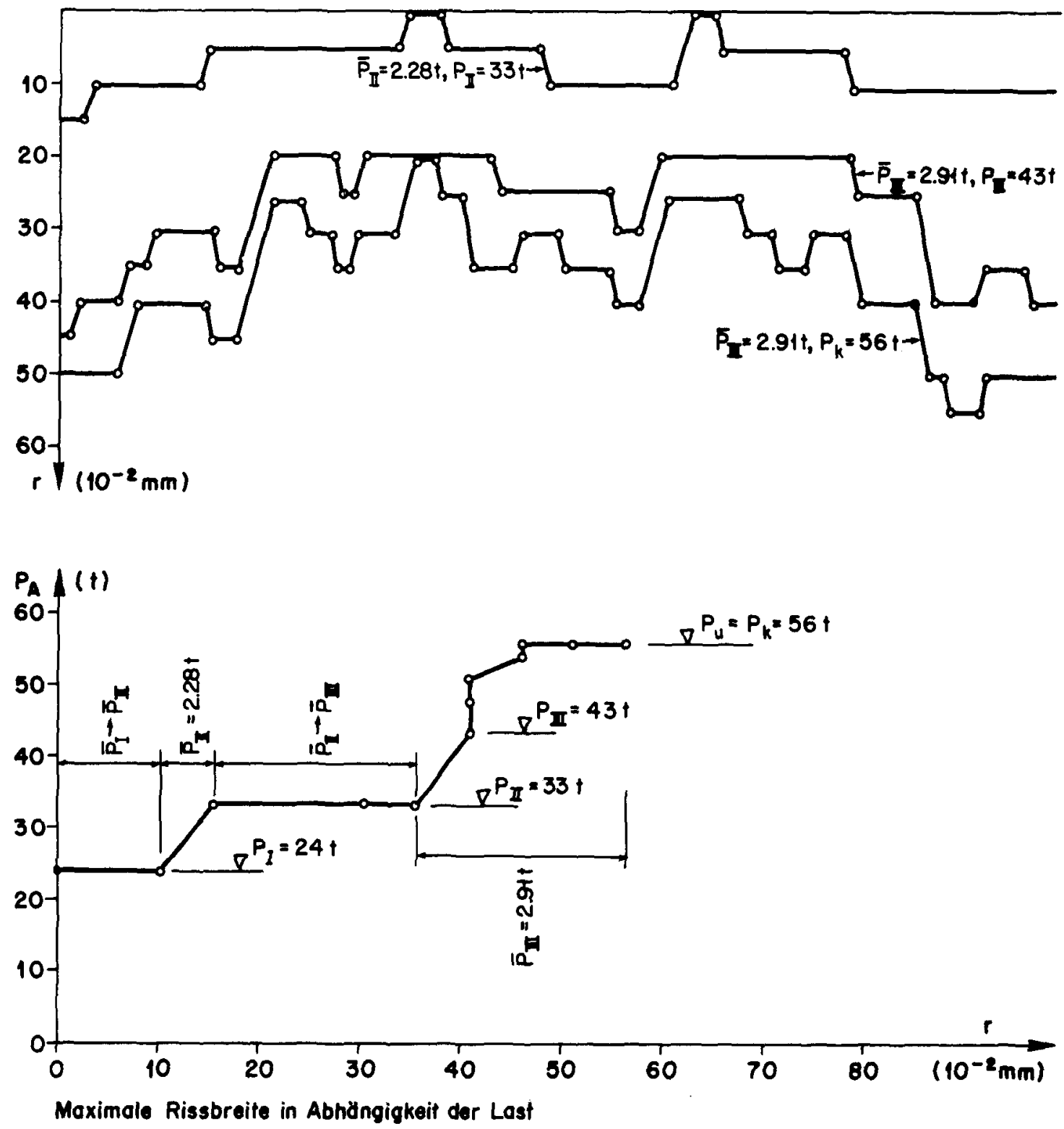

Bild 32: Rissbreiten auf der Plattenoberseite bei Balken $Q 6$ 


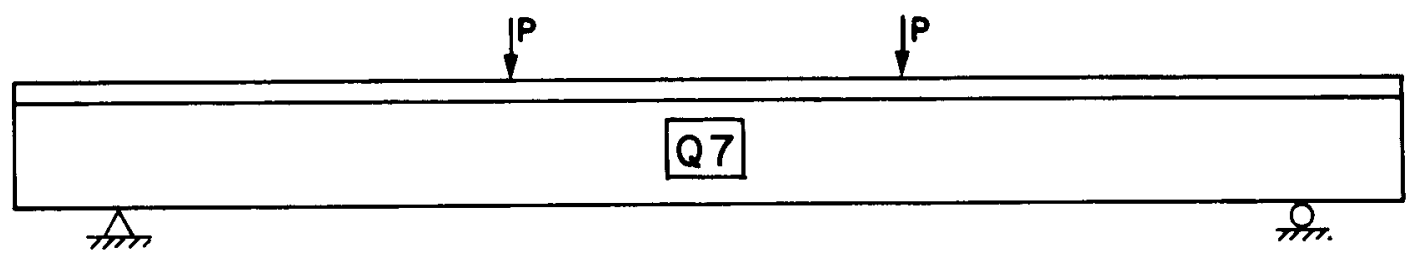

Obere Lage der Plattenarmierung

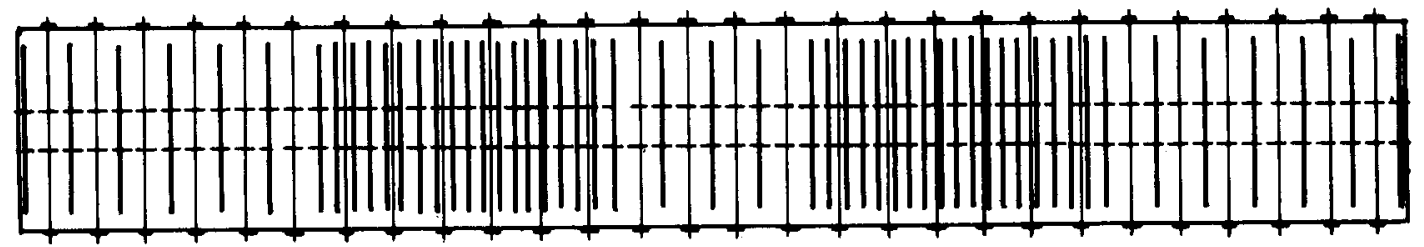

Moximale Risabraite lungs der Platte
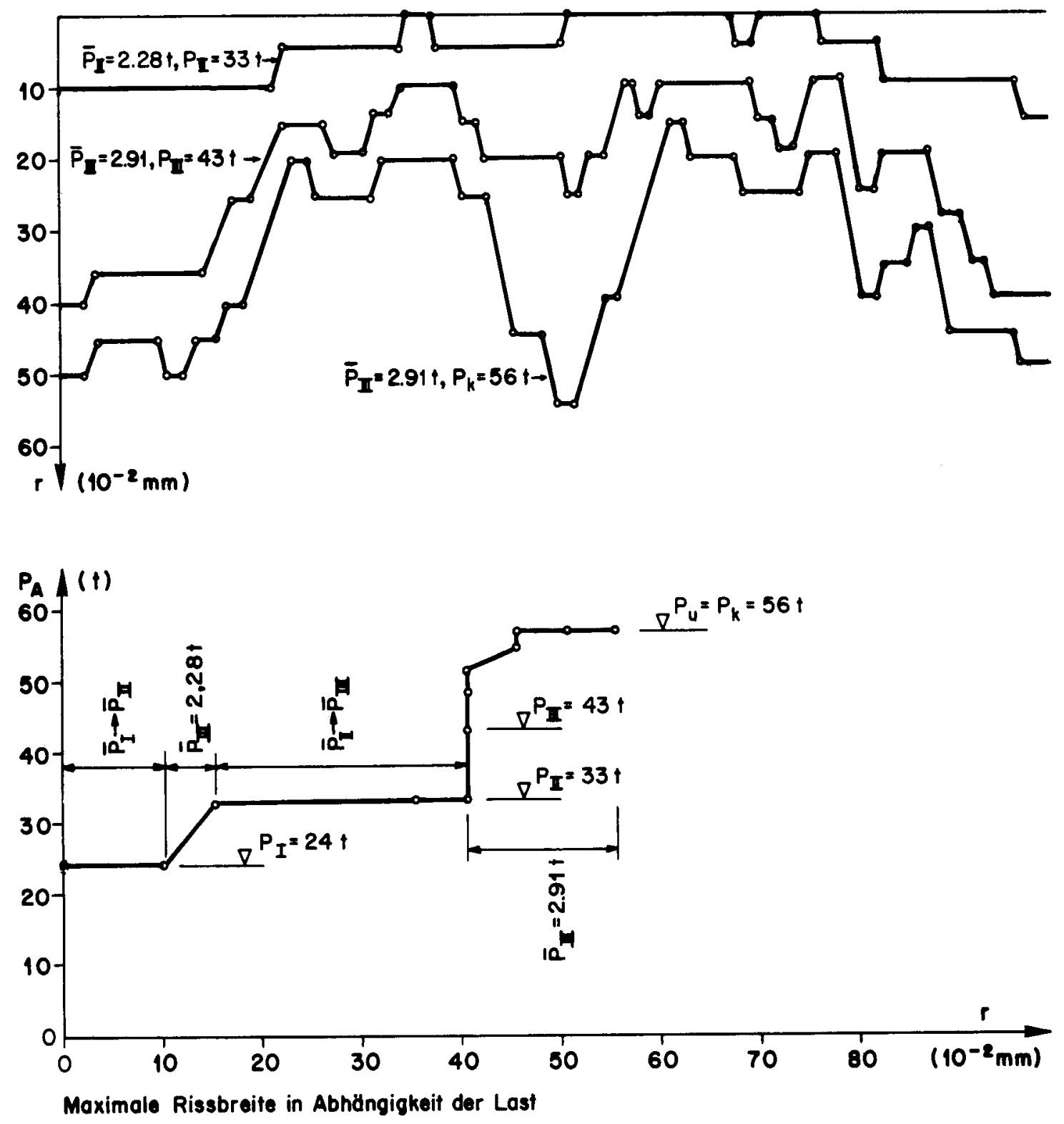

Bild 33: Rissbreiten auf der Plattenoberseite bei Balken Q7 

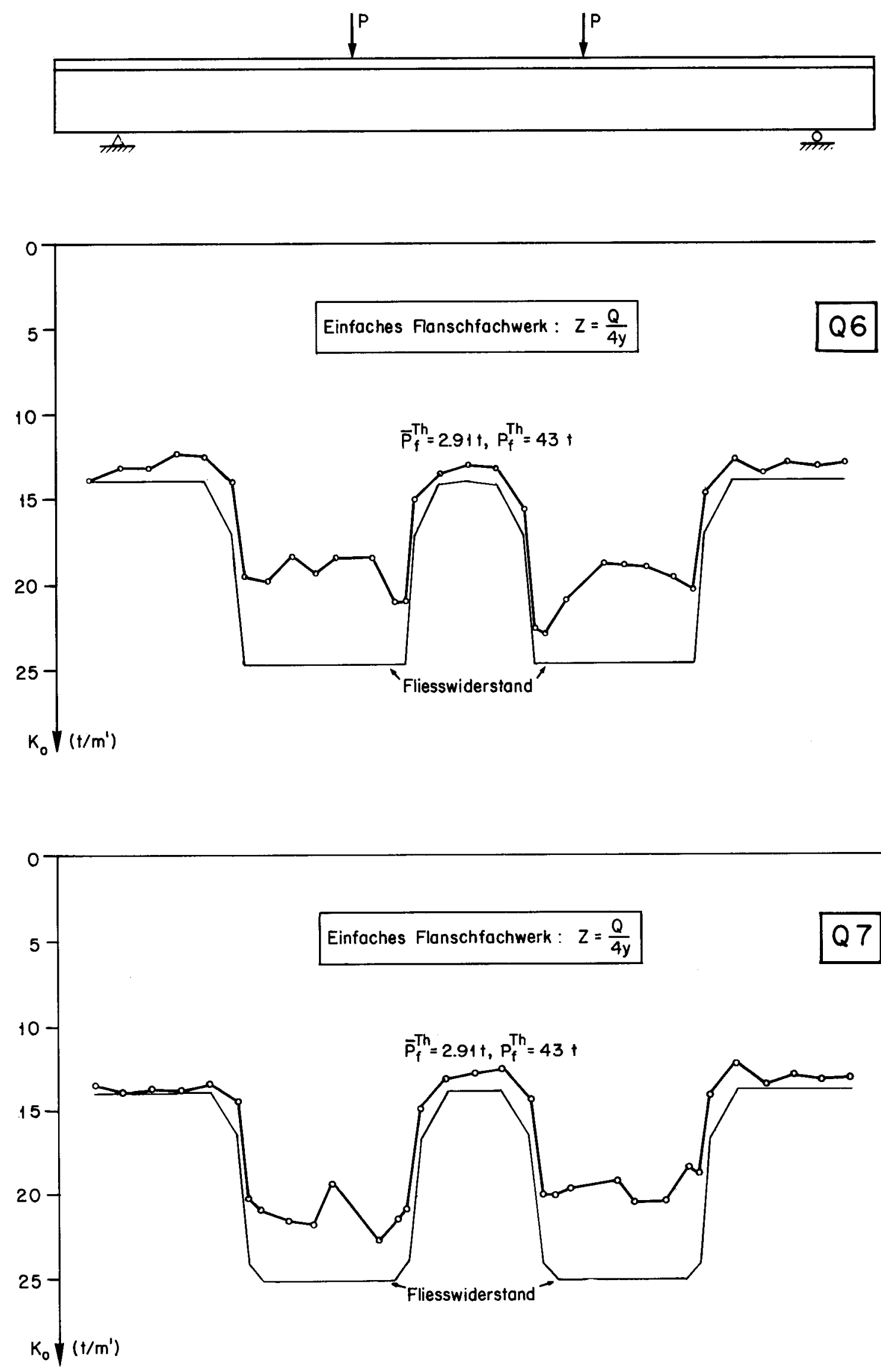

Bild 34: Beanspruchung der oberen Armierungslage unter theor. Fliesslast 

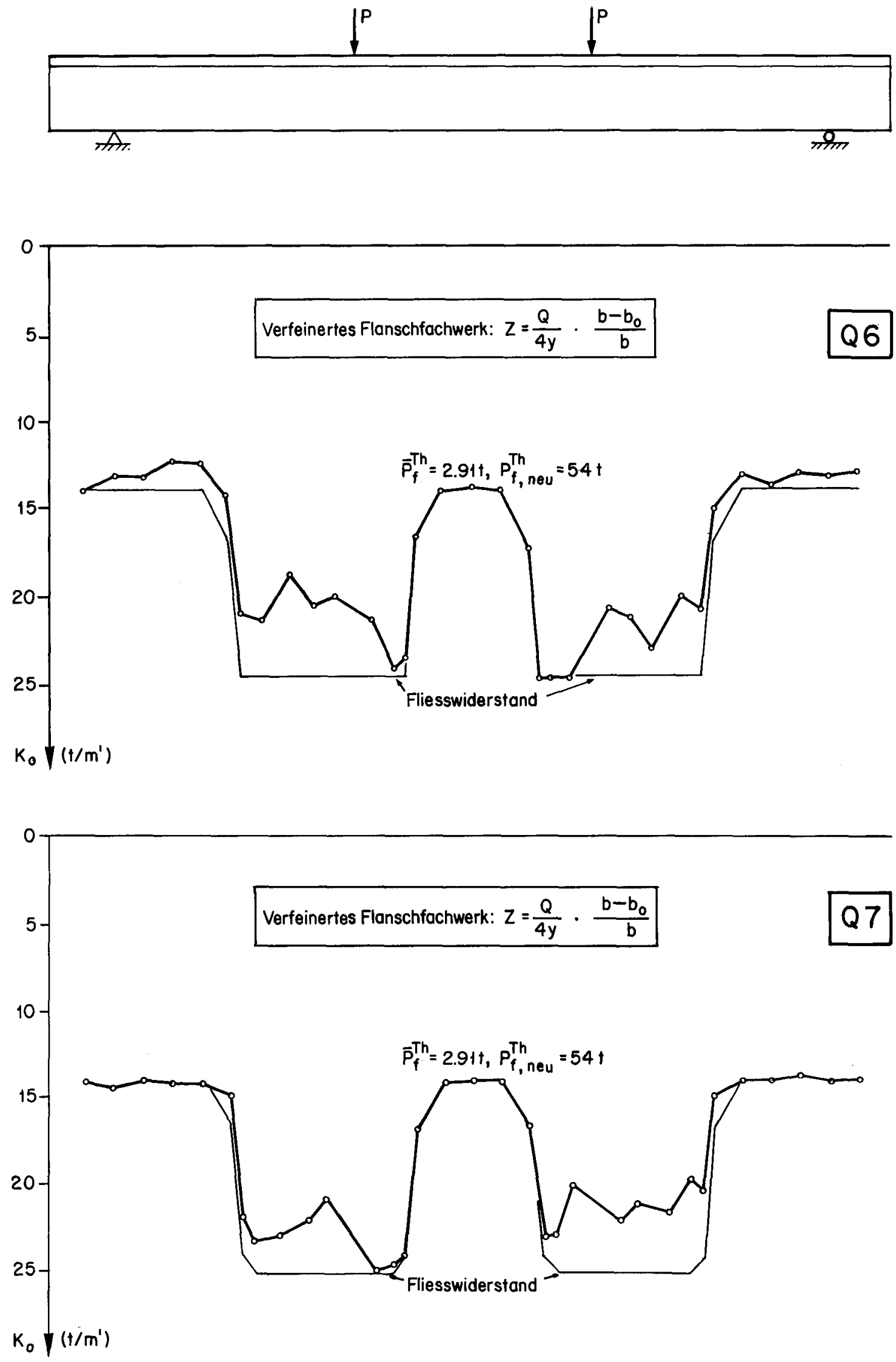

Bild 35: Beanspruchung der oberen Armierungslage unter neuer theor. Fliesslast 


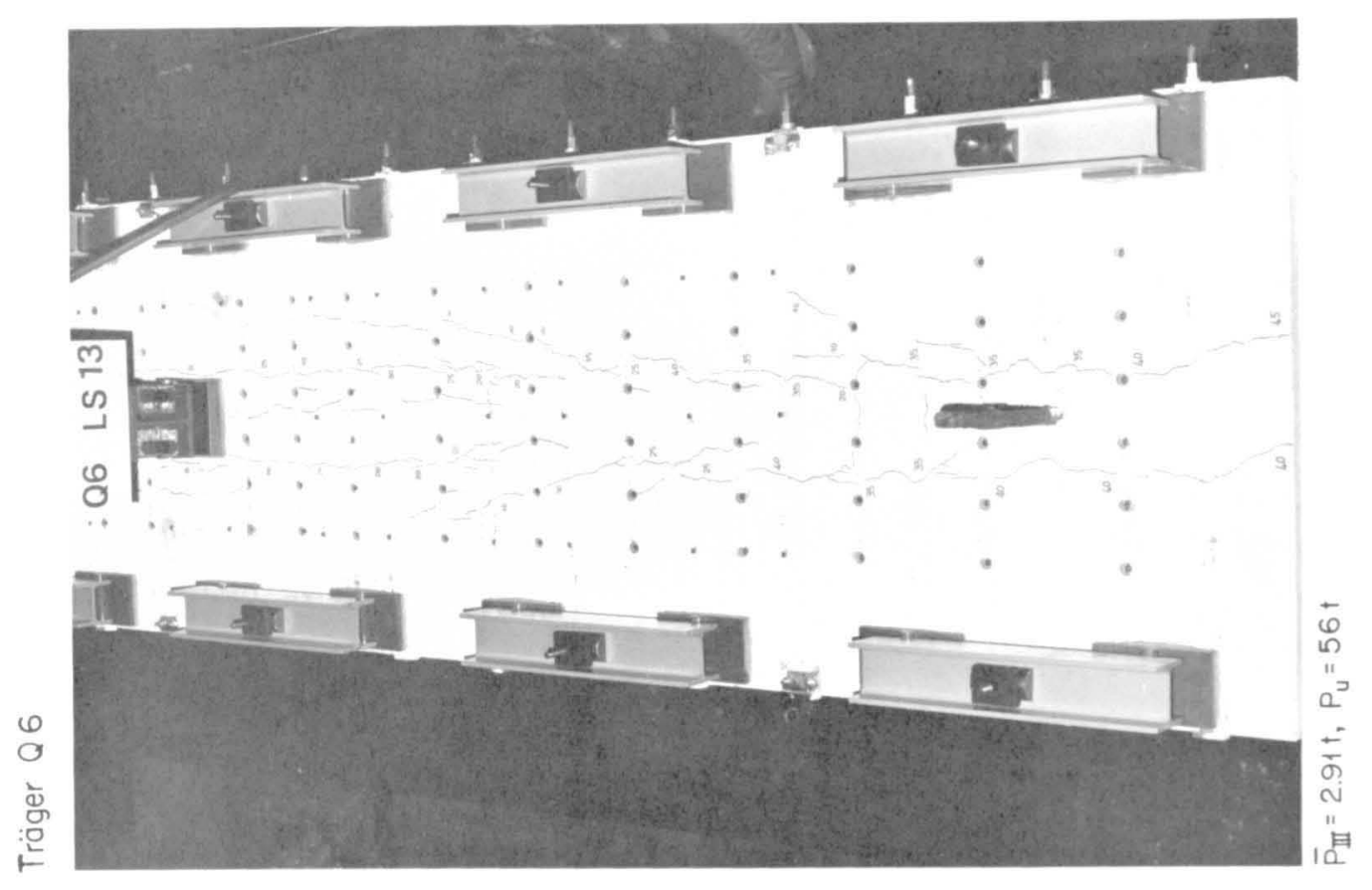

0
0
$\square$
5

$\checkmark$
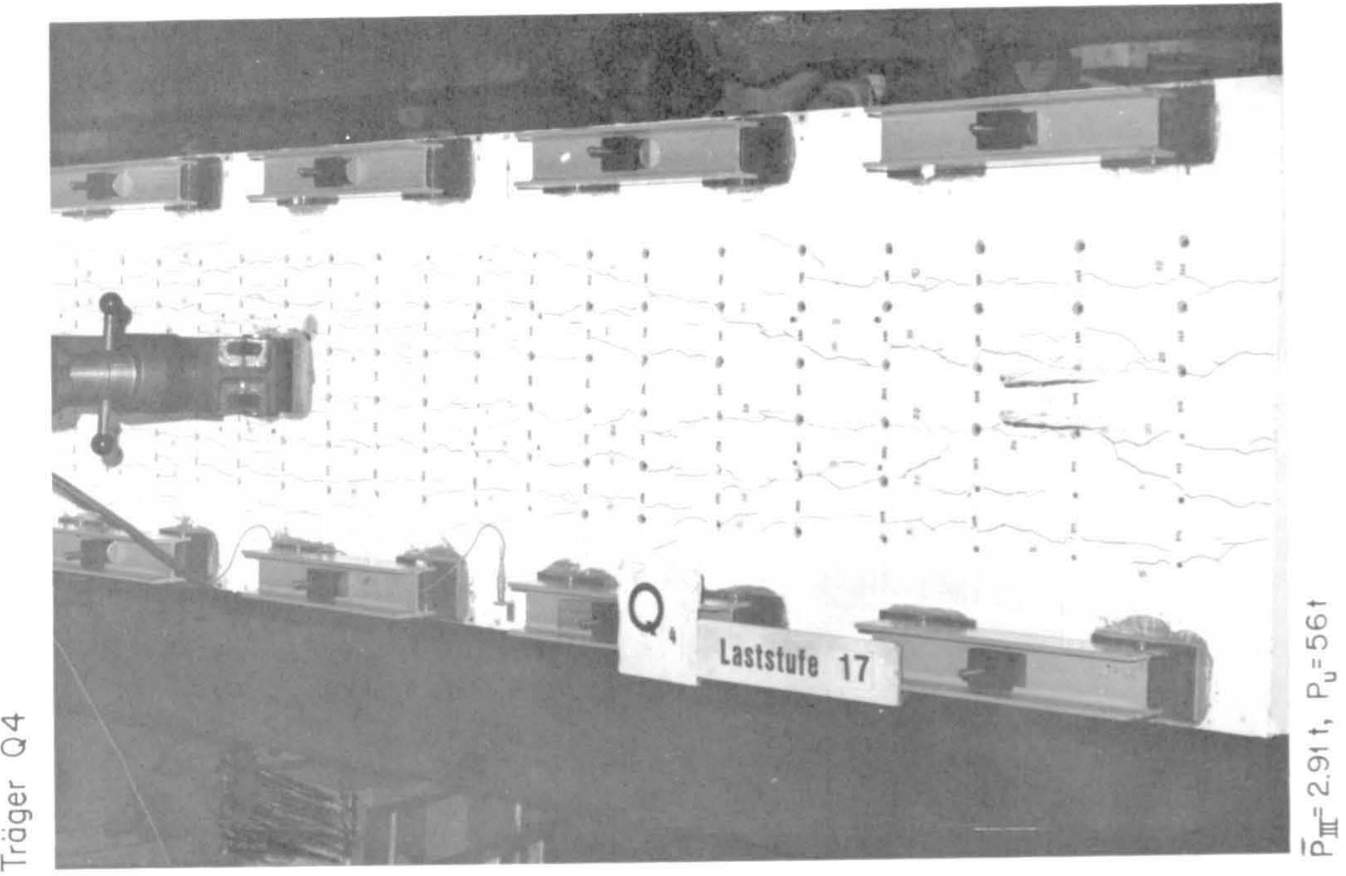

웜

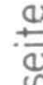

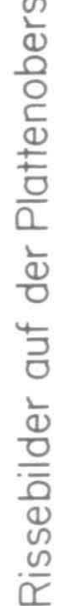

ํํㅁ

$\frac{\frac{1}{0}}{\frac{0}{0}}$

$\dot{m}$

음 

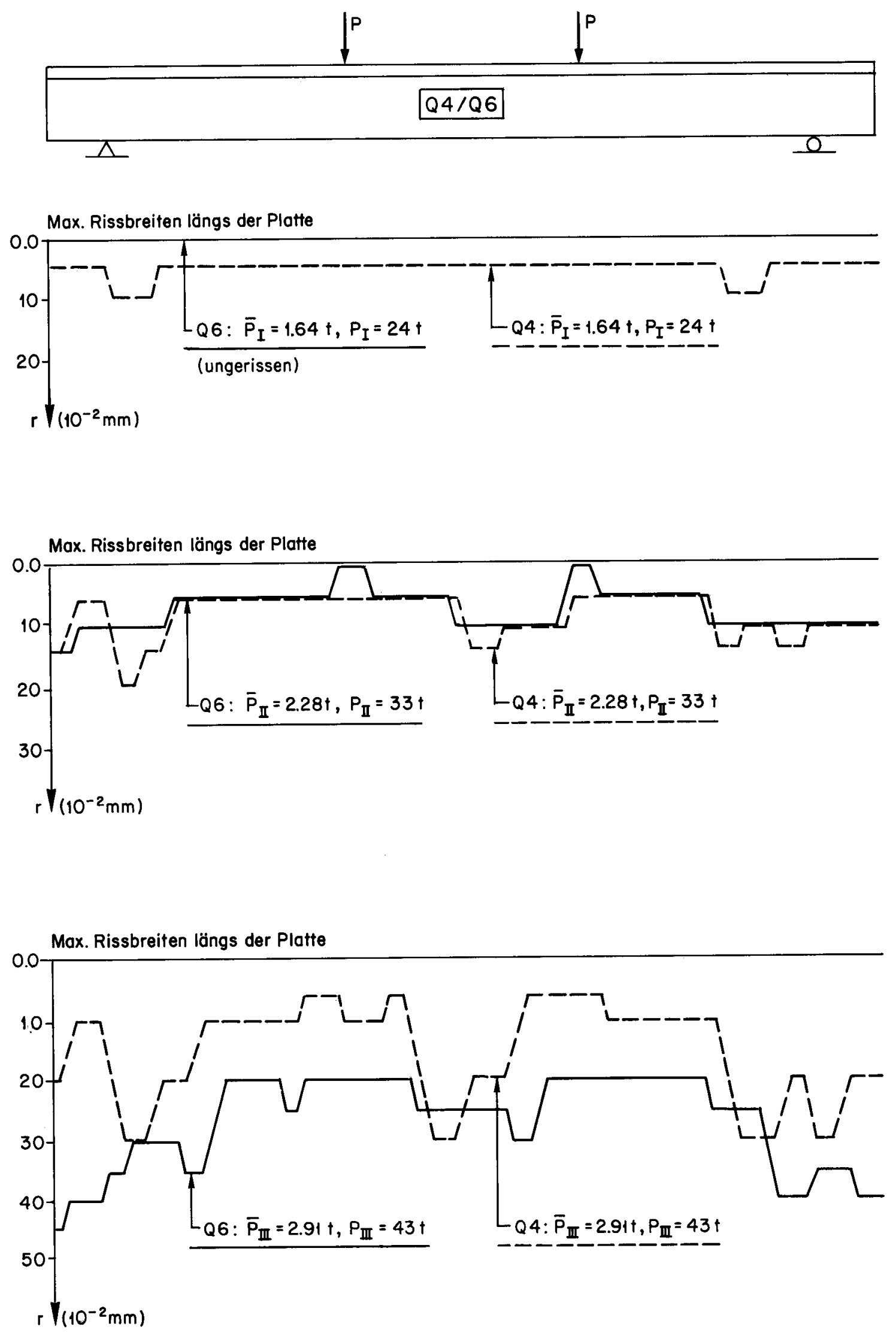

Bild 37: Vergleich der max. Rissbreiten längs der Plattenoberseite der Träger Q4 und Q6 

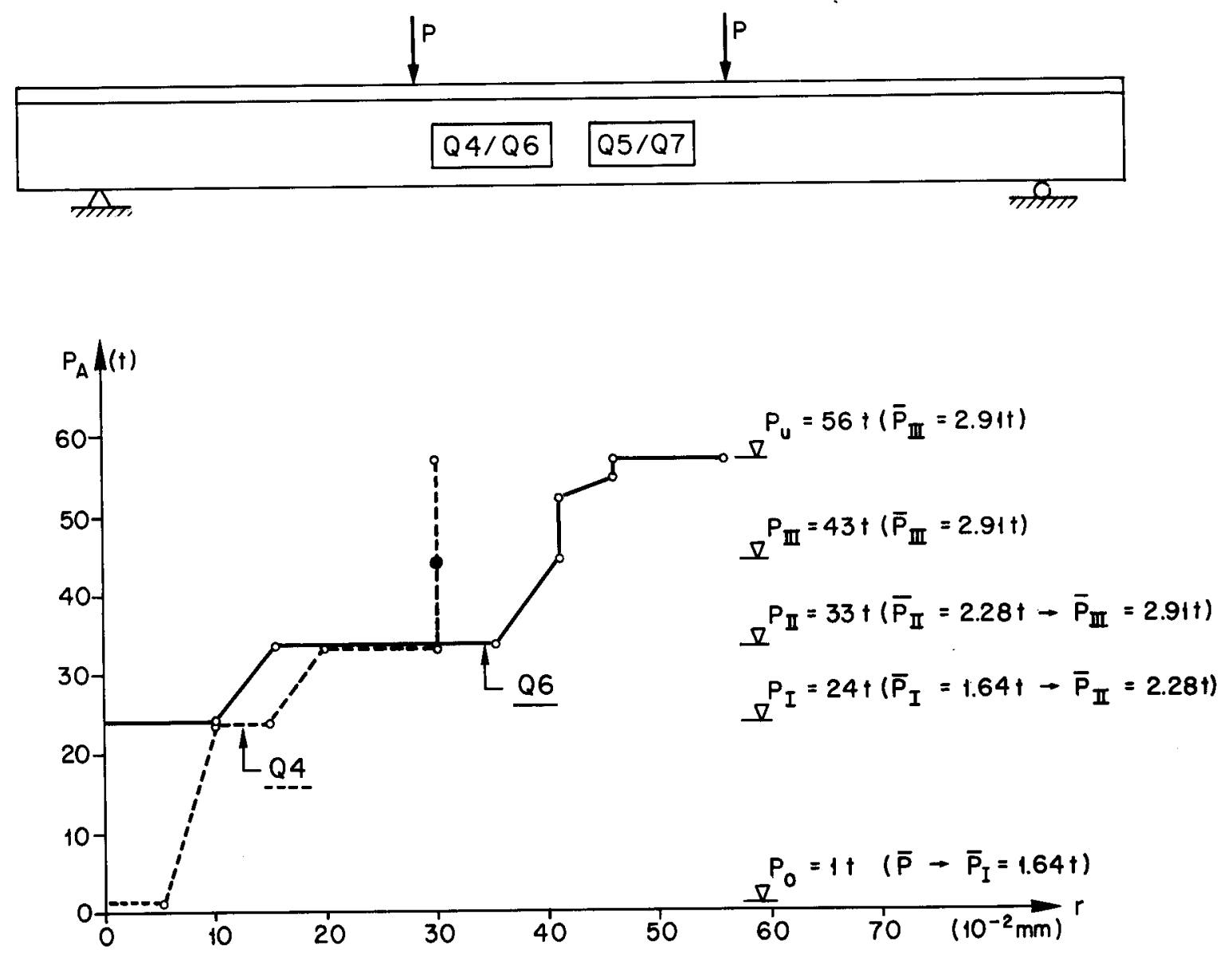

Bild 38-a: Vergleich der max. Rissbreiten (ganze Platte) der Träger Q4 und Q6

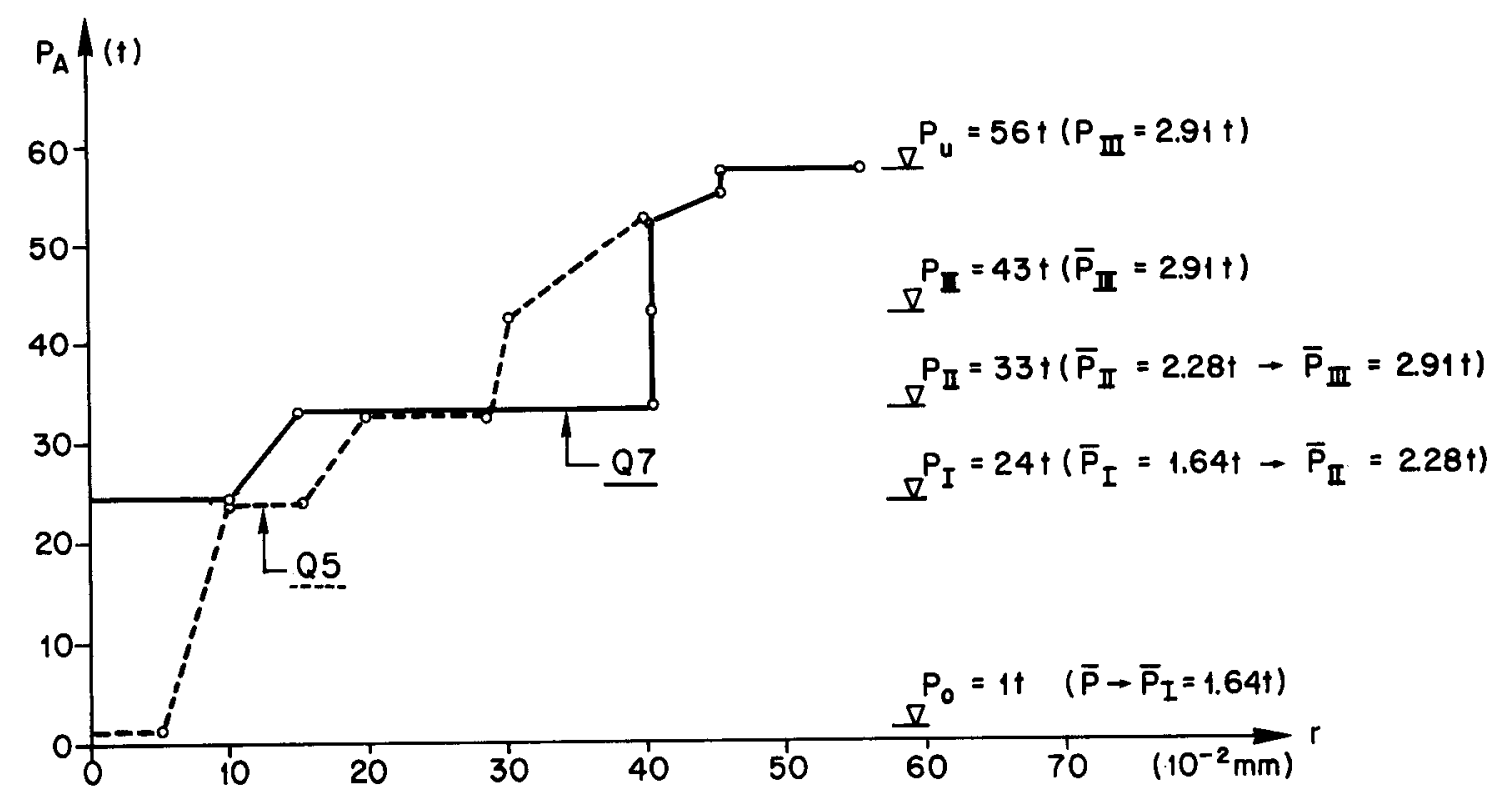

Bild 38-b: Vergleich der max. Rissbreiten (ganze Platte) der Träger $Q 5$ und $Q 7$ 

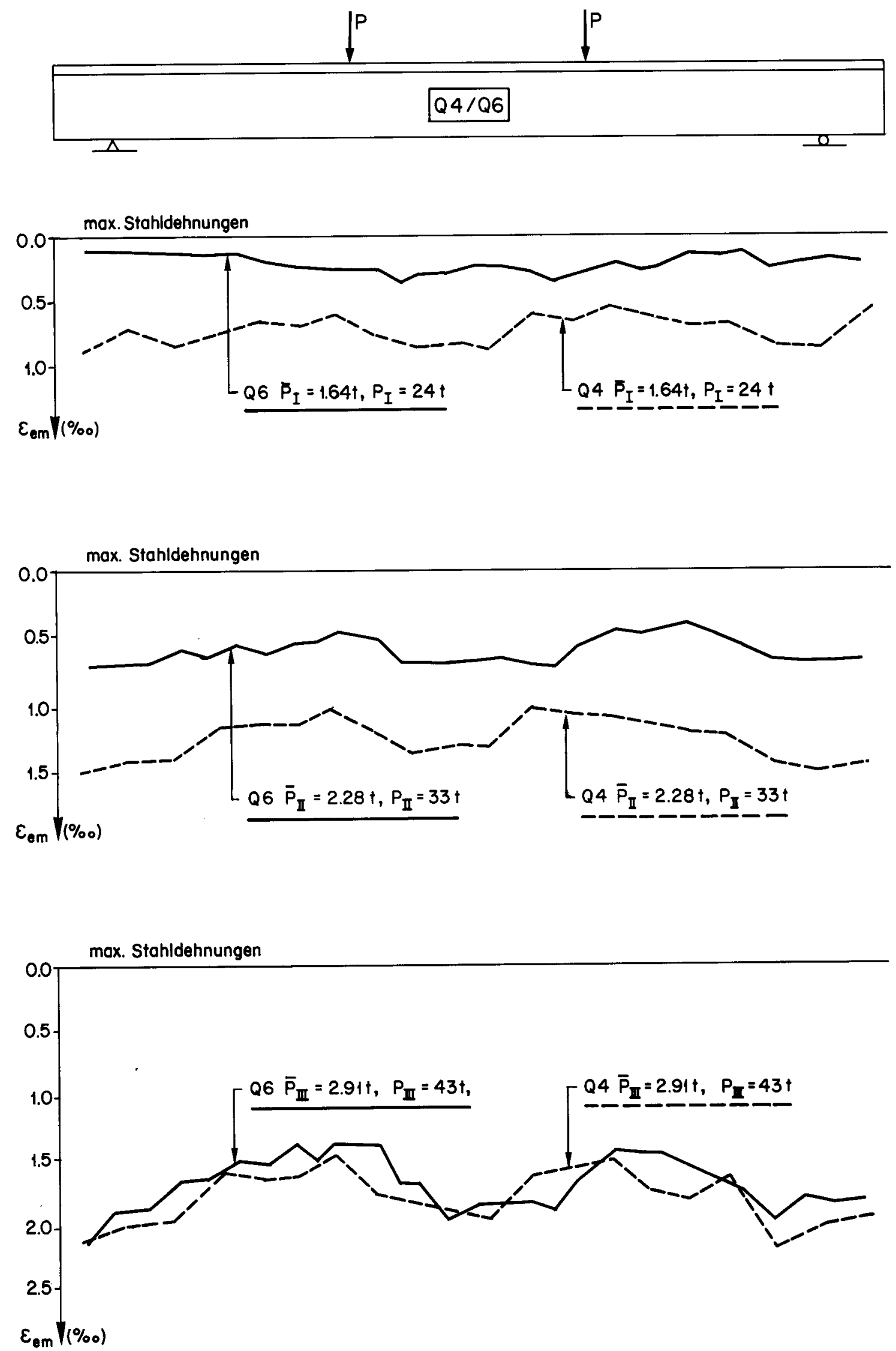

Bild 39: Vergleich der max. Stahldehnungen der oberen Plattenarmierung der Träger Q4 und Q6 



Bild 40-a: Vergleich der Dehnungen an Messstelle 1 der Träger Q4 und Q6

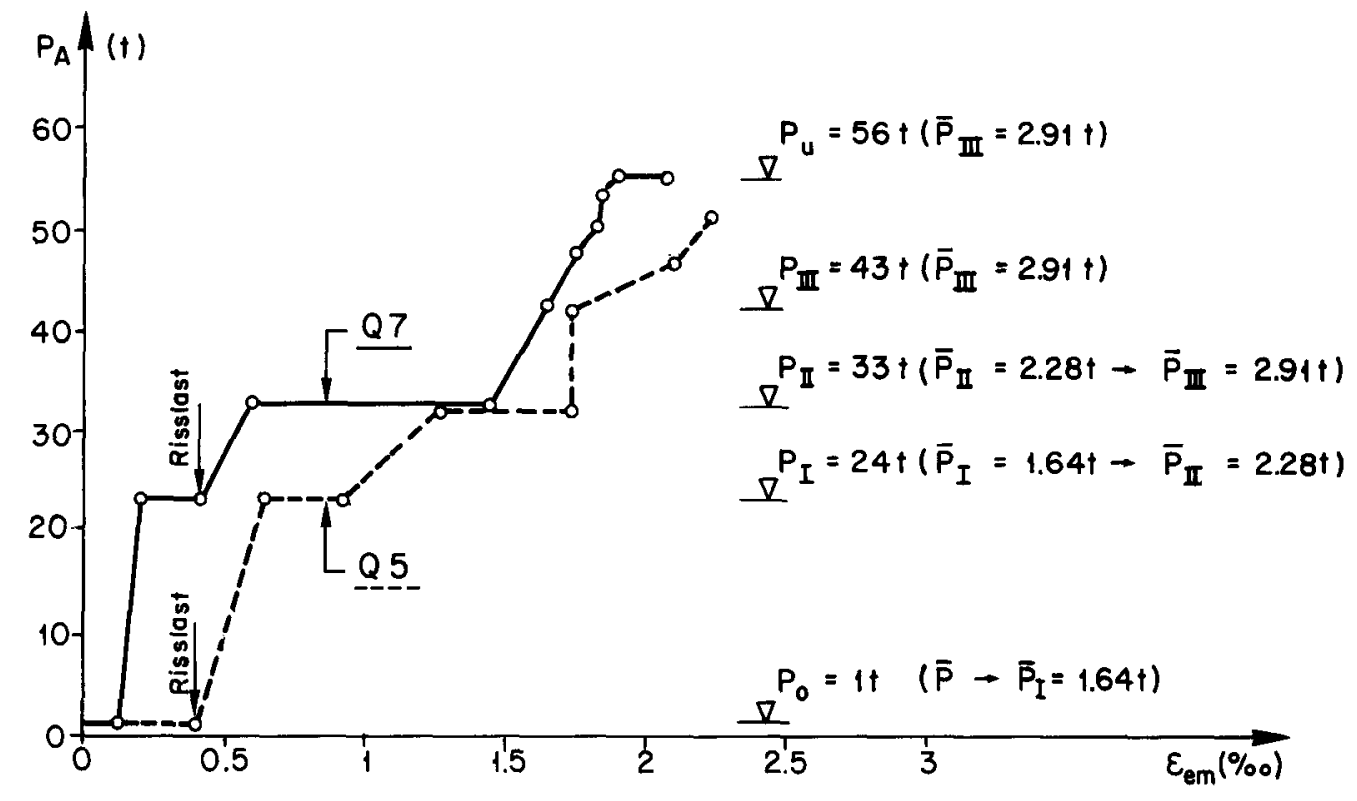

Bild 40-b: Vergleich der Dehnungen an Messstelle 1 der Träger Q5 und Q7 


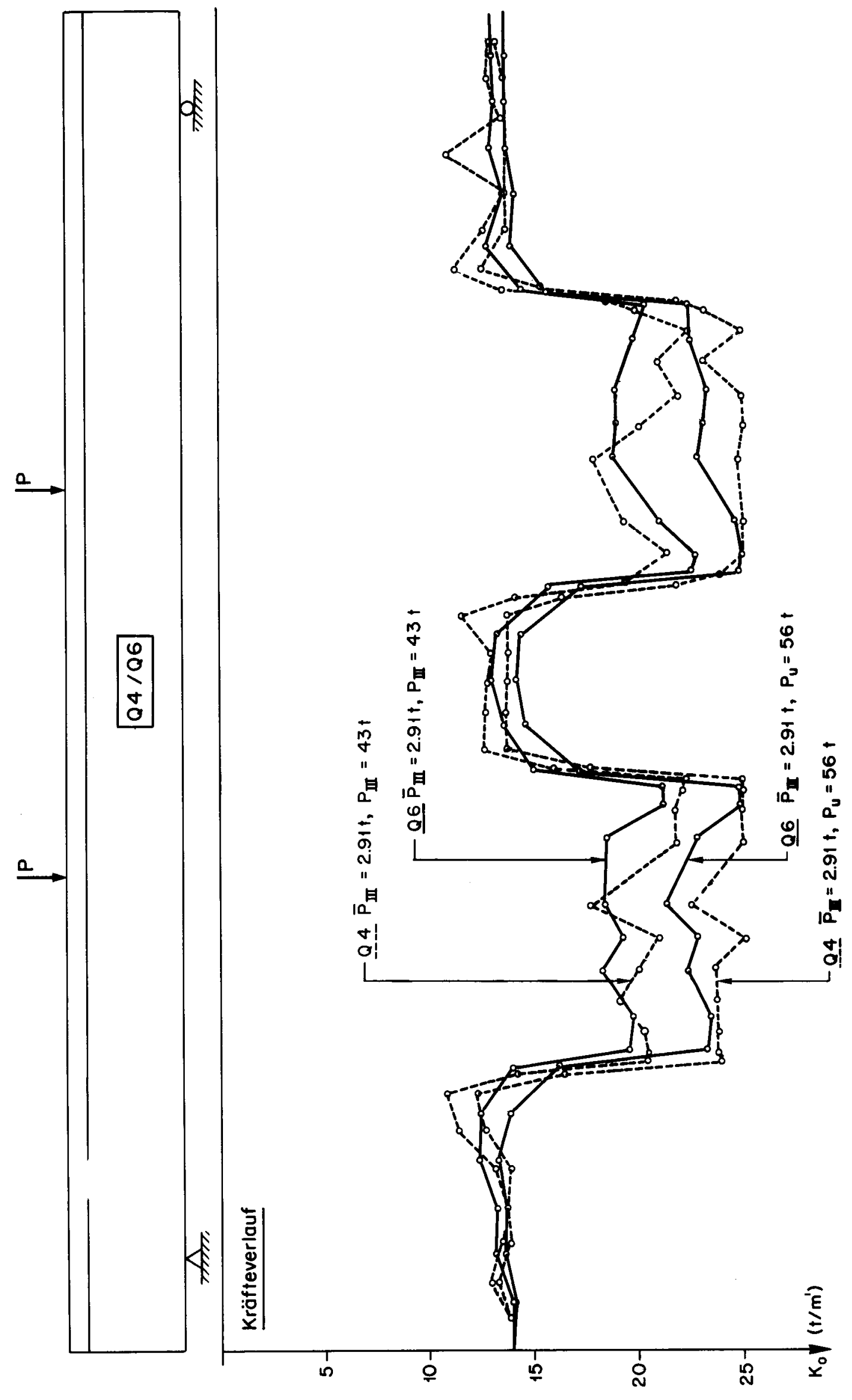

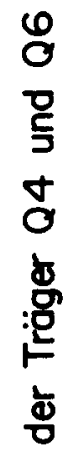

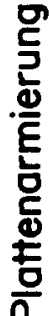

헌

ষ্ํㅁ

드

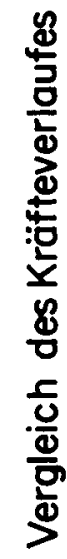

$\ddot{q}$

음 